\title{
First principle-based Research on properties of twisted bilayer of Graphene and GaN
}

\author{
Xiang Cai \\ Submitted to Swansea University in fulfilment of the requirements for the \\ Degree of Doctor of Philosophy
}

College of Engineering, Swansea University

Supervisor: Prof Lijie Li

2021

Copyright: The author, Xiang Cai, 2021. 


\section{Abstract}

Since quantum theory was founded in 1920s, it has developed quite rapidly and led people to a new field that totally different from the classical ones. As the size of an object decrease to a certain level, the classical physical law would be invalid and quantum mechanics would become the ruler of object behaviors. The property under quantum mechanics achieves functions that never been seen before. So, scientist would invent new materials and devices with properties that never found before. This is the reason that new materials and devices have been emerging due to vast developments in nanotechnologies. The project of my PHD research in four years is to research and analysis the property of nano semiconductor materials. In this thesis, there are mainly four parts. The first part is Introduction. In this part the basic concepts and related information would be listed. The second part is the first project that guide me to the further study. This project is set to simulate the electronic transport in quantum region based on the Schrodinger equation for random shaped energy barriers, which is very basic to quantum mechanics. The method of simulation is matlab coding. The simulation will help in understanding the concept of quantum field and applications in design and analysis of nanometer scale devices and systems. The third part is my first paper. In this paper I cooperate with my workmate Shuo Deng and we investigate the electron transport and thermoelectric property of twisted bilayer graphene nanoribbon junction (TBGNRJ) in $0^{\circ}, 21.8^{\circ}, 38.2^{\circ}$ and $60^{\circ}$ rotation angles by first principles calculation with Landauer-Buttiker and Boltzmann theories. It is found that TBGNRJs exhibit a strong reduction of thermal conductance compared with the single graphene nanoribbon (GNR) and negative differential resistance (NDR) in $21.8^{\circ}$ and $38.2^{\circ}$ rotation angles under $\pm 0.2 \mathrm{~V}$ bias voltage. More importantly, three peak $Z T$ values of $2.0,2.7$ and 6.1 can be achieved in the $21.8^{\circ}$ rotation angle at $300 \mathrm{~K}$. The outstanding $Z T$ values of TBGNRJs are interpreted as the 
combination of the reduced thermal conductivity and enhanced electrical conductivity at optimized angles. The fourth part is my second paper. In this paper I report electronic and optical properties of the GaN bilayer structures that are rotated in plane at several optimized rotation angles by using the density functional theory method. For the aim of maintaining the structural stability and using a small cell size, the twisting angles of the GaN bilayer structures are optimized to be $27.8^{\circ}, 38.2^{\circ}$ and $46.8^{\circ}$ using the crystal matching theory. The last part is conclusion, possible further study and acknowledgement. 


\section{Declaration and Statement}

I declare that:

1. the work has not previously been accepted in substance for any degree and is not being concurrently submitted in candidature for any degree.

2. the thesis is the result of my own investigations, except where otherwise stated and that other sources are acknowledged by footnotes giving explicit references and that a bibliography is appended.

3. I give consent for the thesis, if accepted to be made available online in the University's Open Access Repository and for inter-library loan, and for the title and summary to be made available to outside organisations (unless a bar is in place).

4. the University's ethical procedures have been followed and, where appropriate, that ethical approval has been granted.

17, June 2021 
FIRST PRINCIPLE-BASED RESEARCH ON PROPERTIES OF TWISTED

BILAYER OF GRAPHENE AND GAN

ABSTRACT

DECLARATION AND STATEMENT

CHAPTER1: INTRODUCTION

1.1 FIRST PRINCIPLE-BASED CALCULATION

1.2 DENSITY FUNCTIONAL THEORY (DFT) THEORY. 8

1.3 ATOMIC-SCALE MODELING FOR SEMICONDUCTOR \& MATERIALS RESEARCH............. 14

1.4 THERMOELECTRICS .15

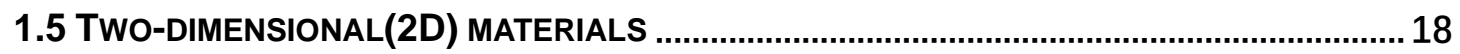

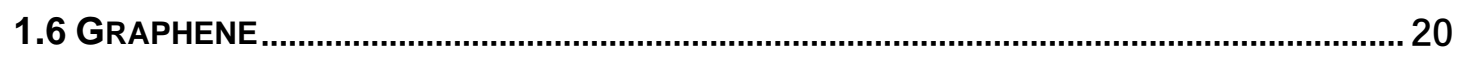

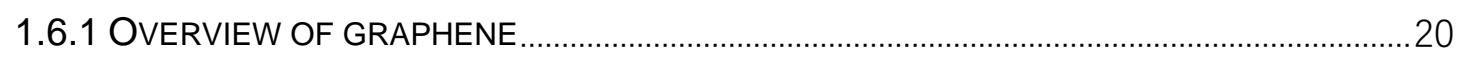

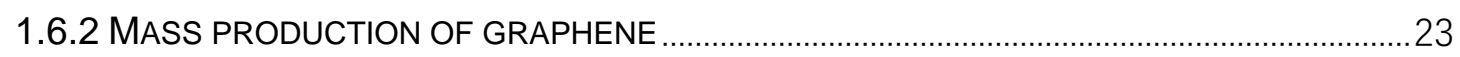

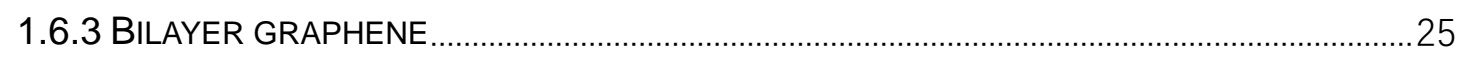

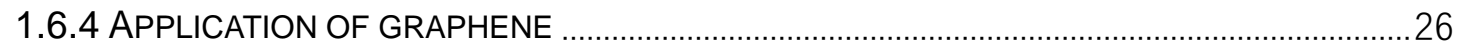

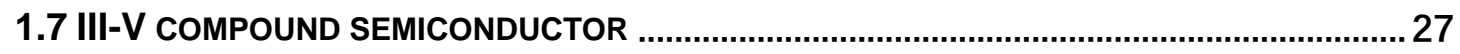

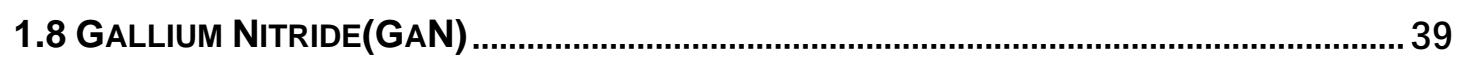

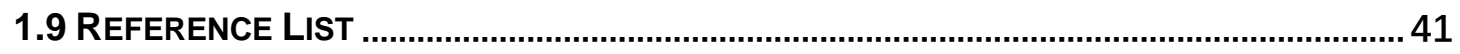

CHAPTER 2: CALCULATION OF THE TRANSMISSION PROBABILITY AND

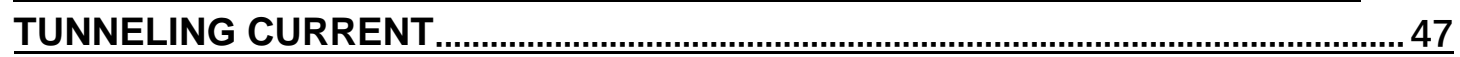

2.1 INTRODUCTION ...................................................................................................... 47

IN ORDER TO ACHIEVE THE GOAL OF THIS PROJECT, SOME CONCEPT MUST BE

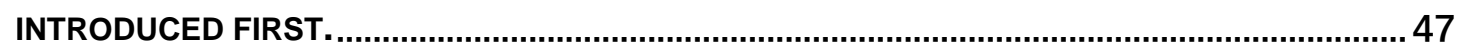

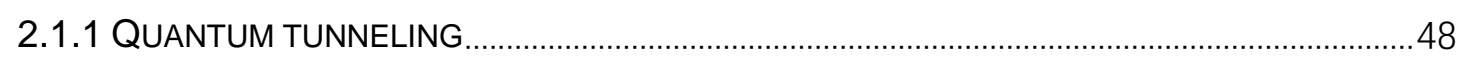

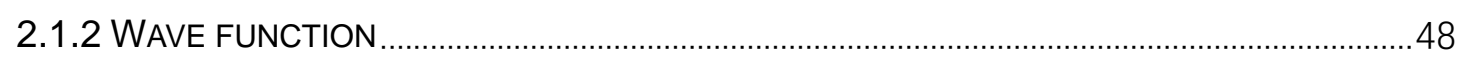

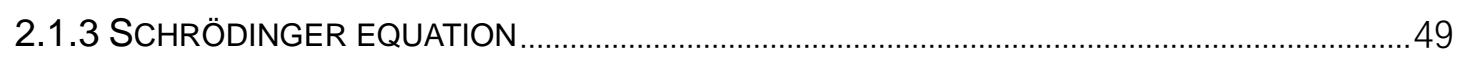

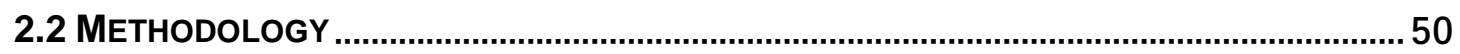

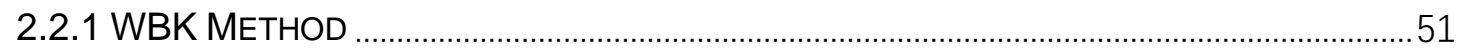

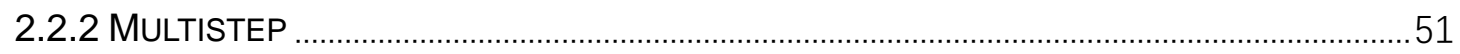

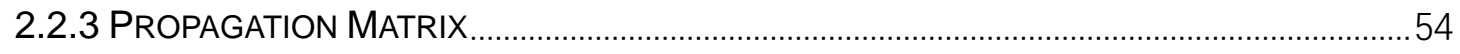

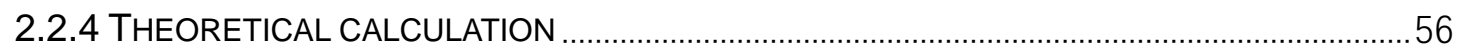

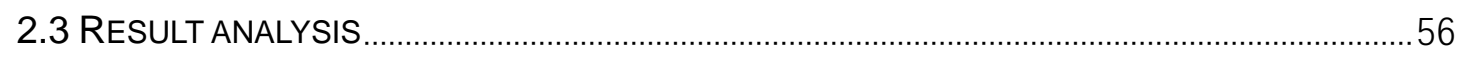

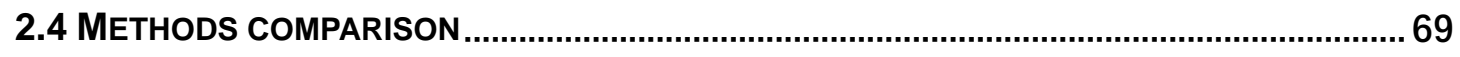

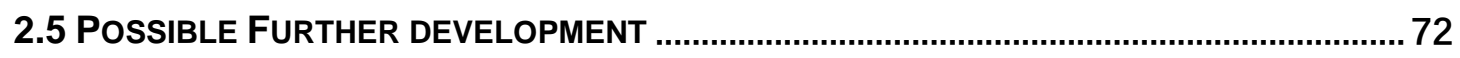


CHAPTER3: STUDY ON THERMOELECTRIC PERFORMANCE OF TWISTED BILAYER GRAPHENE NANORIBBONS JUNCTION BY USING FIRST PRINCIPLE THEORY

3.1 INTRODUCTION

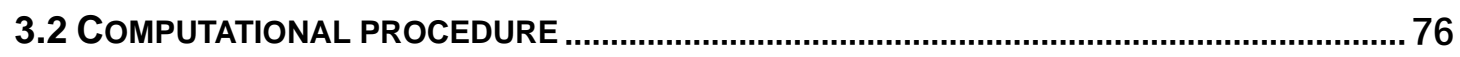

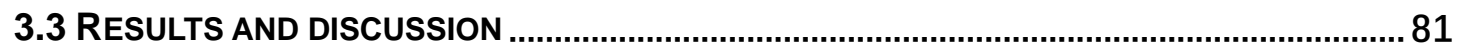

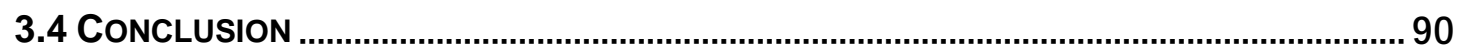

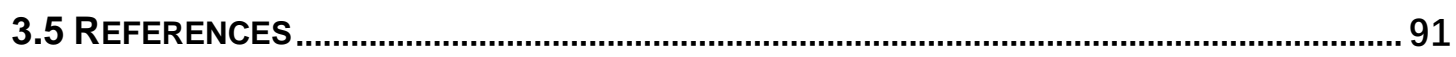

CHAPTER4: STUDY OF ELECTRONIC AND OPTICAL PROPERTIES OF TWISTED BILAYER GAN STRUCTURES BY USING FIRST PRINCIPLES THEORY

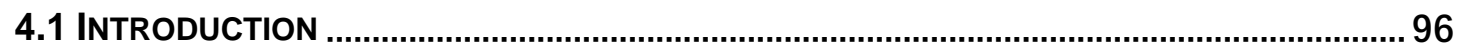

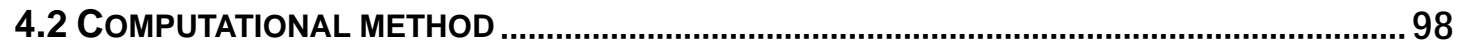

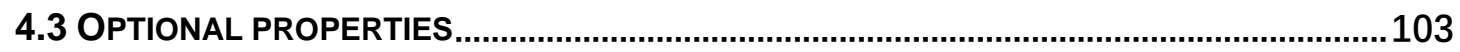

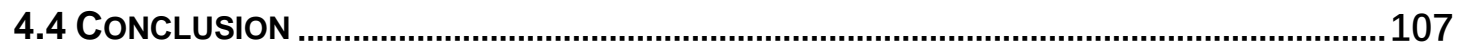

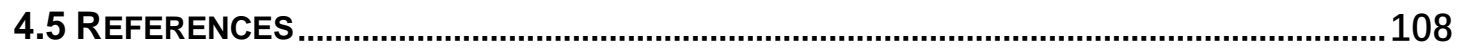

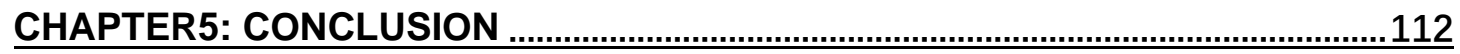

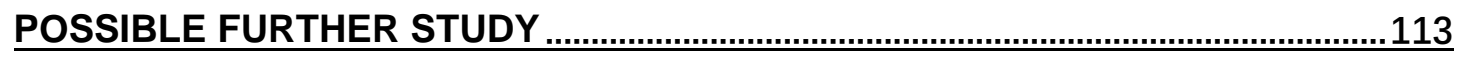

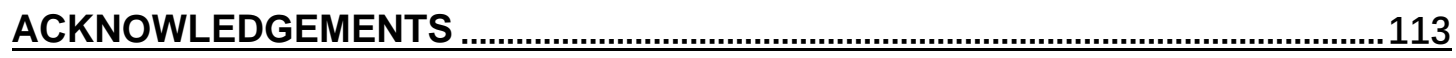




\section{Chapter1: Introduction}

In order to Introduce the result of of my PHD search, some concepts should be brought first. In this part, the following concepts and related application would be listed:

\subsection{First principle-based Calculation}

First-principles is a term for computational physics or computational chemistry.[1] Widely defined first-principles computing refers to calculations based on the principles of quantum mechanics from the very beginning and only based on known theory or principles.

As all known, matter consists of molecules, molecules consist of atoms, and atoms consist of nuclei and electrons. Quantum mechanics calculation is to calculate the molecular structure and molecular energy (or ion) according to the interaction principle of the atomic nucleus and electron, and then can calculate the various properties of the substance. [2-5]

The generalized first principle refers to ab initio. ab initio calculation bases on Hartree-Fock self-consistent field calculation, and density functional theory (DFT) calculation. [6]lt refers to using no experimental parameters, only using a few experimental data such as electron mass, speed of light, and proton neutron mass to do quantum calculations. So, by using first principle calculation, the basic property of unknown material could be speculated. [7]However, this calculation is very slow. In order to speed up the calculation, adding some empirical parameters could be done, but the accuracy of the calculation results would be inevitably sacrificed.[8] So It is important to keep a balance on speed and accuracy 


\subsection{Density functional theory (DFT) theory}

In my four years' learning, the most frequently used method is Density Functional Theory (DFT). Density functional theory (DFT) is a quantum mechanical method for studying the electronic structure of multi-electron systems. Density functional theory has a wide range of applications in physics, materials science, quantum chemistry and life sciences, especially to study the properties of molecules and condensed matter. It is one of the most commonly used methods in the field of condensed matter physical computational materials and computational chemistry. [9-11]

The classical methods of electronic structure theory, especially the HartreeFock method and the post-Hartree-Fock method, are based on complex multielectron wave functions. [12] The main goal of density functional theory is to replace the wave function with electron density as the basic quantity of research. Because the multi-electron wave function has $3 \mathrm{~N}$ variables $(\mathrm{N}$ is the number of electrons, each electron contains three spatial variables), and the electron density is only a function of three variables, regardless of concept It is actually more convenient to deal with.

Although the concept of density functional theory originated from the ThomasFermi model, it did not have a solid theoretical basis until the Hohenberg-Kohn theorem was proposed. Hohenberg-Kohn's first theorem states that the ground state energy of a system is only a functional of electron density.[13] Hohenberg-Kohn's second theorem proves that the ground state energy is obtained by taking the ground state density as a variable and obtaining the minimum value through variation of the system energy.

The HK theory was initially applicable only to ground states where no magnetic field exists, and it has now been generalized. The original Hohenberg-Kohn theorem only pointed out the existence of a one-to-one correspondence but did not provide any such precise correspondence. It is in these exact 
correspondences that there is an approximation. [14]

The most common application of density functional theory is achieved by the Kohn-Sham method. In the framework of Kohn-Sham DFT, the complex multibody problem (due to the interaction of electrons in an external electrostatic potential) is reduced to a problem of non-interacting electrons moving in the effective potential field. This effective potential field includes the effects of external potential fields and Coulomb interactions between electrons, such as exchange and correlation. It is the difficulty of KS DFT to deal with the exchange correlation. At present, there is no accurate solution to the exchange correlation energy Exc. The simplest approximate solution method is local density approximation (LDA). LDA approximates the exchange energy of the system with uniform electron gas (the exchange energy of uniform electron gas can be accurately solved) and uses the method of fitting free electron gas to deal with the correlation energy. [15-16]

Since 1970 , density functional theory has been widely used in the calculation of solid-state physics. In most cases, compared with other methods to solve the multi-body problem of quantum mechanics, the density functional theory using local density approximation gives very satisfactory results, and the cost of solidstate computing is lower than that of experiments. Nevertheless, it is generally believed that quantum chemical calculations cannot give sufficiently accurate results. Until the 1990s, the approximations used in the theory were re-distilled into better exchange correlation models. Density functional theory is currently the leading method of electronic structure calculation in various fields. Although the density functional theory has been improved, it is still difficult to describe the intermolecular forces, especially the van der Waals forces, or to calculate the energy gap of semiconductors.

DFT-based material science calculation simulation methods can not only study existing materials, but also predict new materials. The essence of the DFT method is to use the electron density as the carrier of all information in the ground state of the molecule (atom), rather than the wave function of a single 
electron, so that the multi-electron system is transformed into a single-electron problem for solving. Assuming that the number of electrons is $\mathrm{N}$, the number of variables in the wave function is $3 \mathrm{~N}$, and density functional theory reduces the number of variables to three spatial variables, which not only simplifies the calculation process, but also ensures the accuracy of the calculation.

The development of density functional theory can be roughly divided into three stages. The first stage was in 1927. Thomas and Fermi proposed the ThomasFermi model based on the assumption of uniform electron gas in an ideal state. The concept of density functional was introduced for the first time, which became the prototype of the later DFT method.

The starting point of the Thomas-Fermi model is to assume that there is no interaction between electrons and no external force interference. Then the Schrödinger equation of electron motion can be expressed as:

$$
-\frac{h}{2 m} \nabla^{2} \psi(r)=E \psi(r)(1.1)
$$

By solving this we get

$$
\psi_{k}(r)=\frac{1}{\sqrt{V}} \exp (i k \cdot r)(1
$$

Introducing the electron arrangement rule under $0 \mathrm{~K}$, the electron density, the total energy of a single electron and the kinetic energy density of the system are respectively

$$
\begin{gathered}
\rho=\frac{1}{3 \pi^{2}}\left(\frac{2 m}{h^{2}}\right)^{\frac{3}{2}} E_{F}^{\frac{3}{2}}(1.3) \\
T_{e}=\frac{3 E_{F}}{5}(1.4)
\end{gathered}
$$

Introducing the description of the Coulomb potential and the external field between electrons, the total energy expression of the electronic system determined only by the electron density function can be derived [17]

$$
t[\rho]=\rho T_{e}=\frac{3}{5} \frac{h^{2}}{2 m}\left(3 \pi^{2}\right)^{\frac{2}{3}} \rho^{\frac{5}{3}}=C_{k} \rho^{\frac{5}{3}}(1.5)
$$

Although this model simplifies the calculation form and process, it does not consider the interaction between electrons and does not accurately describe the kinetic energy term, so it is not applicable in many systems. However, under 
the inspiration of this novel research idea, related scholars have basically perfected the content of density functional theory in the 1960s after years of hard work, and finally established a strict sense of density functional theory.

(1) Hohenberg-Kohn theorem

The core idea of Hohenberg-Kohn theorem is that all physical quantities in the system can be uniquely determined by variables that only contain electron density, and the realization method is to obtain the ground state of the system through the principle of variation. This theory is mainly aimed at the non-uniform electron gas model and consists of two sub-theorems. i) An electronic system with external potential (potential other than electronic interaction) that ignores spin, its external potential can be uniquely determined by electron density; ii) For a given external potential, the ground state energy of the system is the minimum of the energy functional value. Therefore, the energy functional of the system can be described as:

$$
E(\rho, V)=\int(r) \rho(r) d r+T[\rho(r)]+\frac{e^{2}}{2} \int \frac{\rho(r) \rho\left(r^{\prime}\right)}{\left|r-r^{\prime}\right|} d r d r^{\prime}+E_{x c}[\rho(r)](1
$$

The right side of the equation are the electron potential energy, kinetic energy term, Coulomb interaction between electrons, and exchange correlation potential energy in the external field.

This theorem does not give specific expressions for the electron density function, kinetic energy functional, and exchange-correlation functional, so the specific solution is still impossible.

(2) Kohn-Sham equations

It was not until 1965 that Kohn and Lu Jeu Sham established the Kohn-Sham 
equation and gave the specific description form of each item, so that the density functional theory began to enter the stage of practical application. For the kinetic energy functional, they propose to use the particle kinetic energy functional without mutual influence as an approximate replacement and incorporate the difference between the two into the unknown term of the exchange correlation functional [18].

$$
\left(-\frac{\hbar^{2}}{2 m} \nabla^{2}+v_{e f f}(r)\right) \varphi_{i}(r)=\varepsilon_{i} \varphi_{i}(r)
$$

This eigenvalue equation is the typical representation of the Kohn-Sham equations.

The Kohn-Sham equation gives clear expressions to items other than commutative correlation functionals and incorporates complex action items into this item. At this point, the computational difficulty has been greatly simplified, and all work began to revolve around how to describe the exchange correlation functional. At the same time, the approximate form of the exchange correlation potential directly determines the accuracy of the density functional theory.

Although the Kohn-Sham equation firstly gives us the way to do the calculation, it is still too complex. Therefore, researchers develop approximation method base on the Kohn-Sham equation to reduce the complexity of calculations. There are two famous approximation method: Local Density Approximation and the Generalized Gradient Approximation.

The Local Density Approximation (LDA) method was also proposed by Kohn and Lu Jeu Sham in 1965. The purpose is to approximate the unknown exchange correlation terms so that the DFT method can be used in actual calculations. LDA uses the density function of uniform electron gas to calculate the exchange correlation term of inhomogeneous electron gas. Assuming that the electron density in the system changes very little with space, the exchange correlation term of inhomogeneous electron gas can be expressed as: 


$$
E_{X C}^{L D A}[\rho(R)]=\int \rho(r) \varepsilon_{X C}[\rho(r)] d r(1.8)
$$

where $\rho$ is the electronic density and $\varepsilon_{x c}$ is the exchange-correlation energy per particle of a homogeneous electron gas of charge density $\rho$.

The corresponding exchange correlation potential can be expressed as

$$
V_{X C}^{L D A}(r)=\frac{\delta E_{X C}^{L D A}}{\delta \rho(r)}=\varepsilon[\rho(r)]+\frac{\delta \varepsilon_{X C}(\rho)}{\delta \rho} \rho(r)
$$

In order to calculate the actual material system more accurately, in 1986, Becke, Perdew and Wang et al. proposed the Generalized Gradient Approximation (GGA), which is currently the most widely used processing method in density functional calculations.

The GGA processing method is to rewrite the original expression term into a functional form that includes the electron density and gradient function, plus the description of the spin, and the obtained exchange correlation functional is as follows:

$$
E_{X C}^{G G A}(\rho)=\int f_{X C}\left(\rho_{\alpha}(r), \rho_{\beta}(r), \nabla \rho_{\alpha}(r), \nabla \rho_{\beta}(r)\right) d r(1.10)
$$

In GGA, exchange correlation potential can also be broken down into exchange energy and correlation energy. Perdew et al. advocate returning to the pure quantum mechanics calculation theory as much as possible, and all physical quantity calculations are only Starting from basic constants such as the static mass of electrons, Planck's constant, and the speed of light, functional expressions should not contain too many empirical parameters, such as GGAPBE (Perdew-Burke-Enzerhoff), which is used in most of my calculations.

The electronic band structure theory based on the Kohn-Sham single-particle energy spectrum can qualitatively describe many materials, but it is not satisfactory from a quantitative perspective. For example, for simple semiconductor materials such as Si and GaAs, the band gap given by KohnSham DFT under LDA/GG is far too small; for small band gap semiconductors 
such as Ge and InN, metal is obtained from LDA/GGA However, what is observed experimentally is a semiconductor, which is the so-called band gap problem of LDA/GGA.

In order to overcome the band gap problem, people have made a lot of efforts within the theoretical framework of DFT, such as extending the Kohn-Sham theory based on the local effective potential to the Generalized Kohn-Sham (GKS) theory based on the non-local effective potential, and Other hybrid density functional theory, as well as a many-body perturbation theory based on one-body Green's function. In this theory, the exchange-correlation self-energy operator corresponds to the exchange-correlation potential of Kohn-Sham DFT. For the self-energy operator, a relatively simple and accurate approximation is the GW approximation (the product of the single-particle Green's function G and the shielded Coulomb effect W). By calculating the self-energy operator under a certain approximation, one can obtain the corresponding PES (IPS) in the excitation energy of the quasi-particles. Although these new development directions have improved the description of the material band gap, the approximate functional still has a lot of subjectivity, and the scope of application is relatively limited. So far, there has not been a universal DFT method with sufficient theoretical basis to accurately describe the electronic energy band structure of materials. $[19,20]$.

\subsection{Atomic-Scale Modeling for Semiconductor \& Materials Research}

In recent years, the exponential growth of computer performance combined with the new development of algorithms has made the practical application of atomic-scale modeling in the design and development of new materials and products in many high-tech industries possible.

A major benefit of atomic modeling is a detailed understanding of atomic-level 
processes, which can complement experimental data and improve the efficiency of the R\&D process through higher quality end results and rapid selection of cost-effective material options. QuantumATK is a complete atom simulation toolkit developed and supported by the world's leading atom-scale modeling experts. QuantumATK solves key applications in the semiconductor industry and is a core component of Synopsys design technology collaborative optimization (DTCO) solutions. The atomic-scale modeling tools in QuantumATK range from classic force fields used to handle large and somewhat more realistic material systems to ab initio tools that provide highly accurate results for smaller systems.

\subsection{Thermoelectrics}

Currently, the world faces many challenges related to energy supply and consumption. On one hand, even with recorded high oil prices, global demand for oil didn't stop to grow. On the other hand, people are paying more and more attention to the environmental impact of greenhouse gases, especially carbon dioxide. All these problems are driving people's demand for more useful energy. For example, many new vehicle engines, transmissions and related technologies are being developed to improve the fuel efficiency of transportation for fuel vehicles. However, these technologies lack an important issue: much of the energy will still generate unusable heat in the fuel vehicle exhaust or cooling system. In fact, in an internal combustion engine, about $40 \%$ of the fuel energy is wasted in the exhaust gas, $30 \%$ of the fuel is dissipated in the engine coolant, $5 \%$ of the heat is lost due to radiation and friction, and only $25 \%$ of the energy can be used for vehicle movement. Current waste energy assessments show that in the United States alone, 46 billion gallons of gasoline are wasted from the exhaust pipes of about 200 million light vehicles each year. 
[21] In addition, the waste heat generated from end-to-end power production losses is much larger, accounting for about two-thirds of the available energy in power plants and manufacturing. In the US manufacturing industry alone, more than 3,000 TW of waste heat energy is lost each year, equivalent to more than 1.72 billion barrels of oil. Therefore, the potential for developing highly efficient thermoelectric materials for waste heat recovery systems is huge. [2225]

The thermoelectric effect is a direct conversion of the voltage generated by the temperature difference, and vice versa. Simply placing a thermoelectric device will produce a voltage when there is a temperature difference between their ends, and when a voltage is applied to it, it will also produce a temperature difference. This effect can be used to generate electrical energy, measure temperature, and cool or heat objects. Because the direction of heating or cooling depends on the applied voltage, thermoelectric devices make temperature control very easy just by changing the voltage applied.

For a long time, the thermoelectric field has been considered as a potential transformative power generation technology. Due to its ability to directly convert heat into electrical energy and develop cost-effective, pollution-free forms of energy conversion, the thermoelectric field is steadily developing. Among various types of thermoelectric materials, nanostructured materials have shown the most promise for commercial use due to their extraordinary thermoelectric properties.[26]

Generally, the term thermoelectric effect here includes three separately defined effects, the Seebeck effect (found by Thomas Johann Seebeck), the Peltier effect (found by Jean-Charles Peltier.), and Thomson effect (Thomson effect, discovered by William Thomson). In this thesis, we only discuss the Seebeck effect. Different metal conductors (or semiconductors) have different free electron densities. When two different metal conductors (or semiconductors) are in contact with each other, the electrons on the contact surface will diffuse to eliminate the difference in electron density. The terminal forms a stable 
voltage, and the diffusion rate of electrons is proportional to the temperature of the contact area. The resulting voltage is usually only a few microvolts per Kelvin temperature difference. The same metal (or semiconductor) at different temperatures also has different free electron densities, so as long as the temperature difference between the two ends of the metal is maintained, the electrons can continue to diffuse, and a stable voltage is formed at the two ends of the metal.

The energy conversion efficiency of thermoelectric materials can be described by the figure of merit $Z T=S^{2} G_{e} T /\left(\kappa_{e}+\kappa_{p h}\right)$, where the $G_{e}, S$ and $T$ are the electrical conductance, Seebeck coefficient and temperature, respectively. $\kappa_{e}$ and $\kappa_{p h}$ are the heat transport coefficient of electrons and phonons. The Seebeck coefficient, which is also known as thermal power, thermoelectric power or thermoelectric sensitivity, is the magnitude of measurement of the induced thermoelectric voltage across the temperature difference caused by the Seebeck effect. A good thermoelectric material should have a high electrical conductivity, Seebeck coefficient and low thermal conductivity. To ensure that the Seebeck coefficient is large, a single type of carrier (n-type or p-type) should be retained, because mixed n-type/p-type charge carriers will cause the opposite Seebeck effect, resulting in lower thermal power. In order to obtain a single type of carrier, it is necessary to choose a material with a suitable energy band gap and a suitable doping, where the n-type and p-type can be well separated. Therefore, an effective thermoelectric material is a heavily doped semiconductor with an energy band gap of less than $1 \mathrm{eV}$ to have both a single carrier type and a sufficiently high carrier mobility. Obviously, decoupling the thermal and electronic terms (including Seebeck coefficient and conductivity) that determine $\mathrm{ZT}$ has become a key to improve $\mathrm{ZT}$. Low-dimensional thermoelectric materials are considered to have higher thermoelectric performance than their large-volume thermoelectric materials, because DOS near the Fermi level can be enhanced by quantum confinement, resulting in an increase in thermal power. And/or because phonons in a large mfp range can 
be effectively scattered through a high-density interface, thus leading to a reduction in lattice thermal conductivity. Significant $\mathrm{ZT}$ enhancements have been found in two-dimensional (2D) and one-dimensional (1D) thermoelectric materials.[27]

Devices based on two-dimensional thermoelectric materials (such as thin films, quantum wells, and superlattices) can be used in small electronic and optoelectronic applications that require small thermal loads or low power generation. However, their thermal and chemical stability related to thermal migration and electromigration has attracted much attention. In particular, due to the great difficulty of integrating nano-scale materials into micro-scale devices and packaged macro-scale systems, a fully functional and practical thermoelectric cooler has not yet been made from these nano-materials.[28,29]

\subsection{Two-dimensional(2D) materials}

Two-dimensional(2D) materials are a general term for a large class of materials. It referring to in one dimension the material size is reduced to the limit, about the thickness of layers of atoms, while in the other two dimensions, the material size is relatively large.[30] The most typical and first experimentally proven twodimensional material is graphene. In 2004, K. S. Novoselov et al. published an article in Science magazine [33], which reported that graphene was obtained from highly oriented pyrolytic graphite by mechanical exfoliation and proved its unique and excellent electrical properties. Since then, two-dimensional materials represented by graphene have developed rapidly, and new twodimensional materials have been discovered. Because of the quantum confinement effect in the thickness direction of the atomic layer, these twodimensional materials exhibit completely different properties from their corresponding three-dimensional structures, and thus have received extensive 
attention from the scientific and industrial communities.

Besides graphene, other two-dimensional materials found today include: single-element silicon, germane, tinene, boron and black phosphorus, etc., transition metal chalcogenide compounds such as $\mathrm{MoS}_{2}, \mathrm{WSe}$, $\mathrm{ReS}_{2}, \mathrm{PtSe}_{2}$, $\mathrm{NbSe}_{2}$, etc. Main group metal chalcogenide compounds such as GaS, InSe, $\mathrm{SnS}, \mathrm{SnS}_{2}$, etc., and other two-dimensional materials such as h-BN, $\mathrm{Crl}_{3}, \mathrm{NiPS}_{3}$, $\mathrm{Bi}_{2} \mathrm{O}_{2} \mathrm{Se}$, etc. These two-dimensional materials have completely different energy band structures and electrical properties, covering material types from superconductors, metals, semi-metals, semiconductors to insulators. At the same time, they also have excellent optical, mechanical, thermal, magnetic and other properties. [31] By stacking two-dimensional materials of different types, a more functional material system can be constructed. Therefore, these materials are expected to be applied in the fields of high-performance electronic devices, optoelectronic devices, spintronic devices, energy conversion and storage.

At this stage, the research on two-dimensional materials focuses on preparation, characterization, modification, theoretical calculation and application exploration, and has made great progress. For example, in terms of preparation, many methods can be applied to satisfy the requirement in different fields. Mechanical lift-off method is widely used to prepare twodimensional material samples for laboratory physical properties research and device fabrication. Chemical vapor deposition method can prepare large-area, high-quality, controllable number of graphene and some transition metal chalcogenide materials. This have laid the foundation for commercial application. Researchers have established a series of characterization methods including complementary spectroscopy and electron transport for the characterization of two-dimensional materials. Modification is also an important aspect of the development of two-dimensional materials. [32] Methods 
including doping, chemical modification, electrostatic control, alloys and other means are applied to avoid the shortcomings of the material itself and extent its advantages. Theoretical calculations play a vital role in the development of two-dimensional materials. Through theoretical calculations, more new twodimensional materials can be discovered, their performance can be predicted, the observed phenomena can be explained, and the experimental design can be guided. In terms of applications, high-frequency transistors based on graphene, short-channel field-effect transistors and tunneling transistors based on $\mathrm{MoS}_{2}$, and the realization of other high-efficiency light-emitting and photo detecting devices have all shown great application potential for twodimensional materials.

\subsection{Graphene}

\subsubsection{Overview of graphene}

Graphene is an atomic-scale honeycomb lattice of carbon atoms.

Graphene is a crystalline allotrope of carbon in the form of a nearly transparent (to visible light) one atom thick sheet. It is hundreds of times stronger than most steels by weight. It has the highest known thermal and electrical conductivity, displaying current densities $1,000,000$ times that of copper. It was first produced in 2004.

Andre Geim and Konstantin Novoselov [33] won the 2010 Nobel Prize in Physics "for groundbreaking experiments regarding the two-dimensional material graphene". They first produced it by lifting graphene flakes from bulk graphite with adhesive tape and then transferring them onto a silicon wafer.

The arrangement of carbon atoms in graphene is the same as the single-layer graphite layer. The bonds are sp2 hybrid orbits and have the following 
characteristics: carbon atoms have 4 valence electrons, of which 3 electrons generate sp2 bonds. This means each carbon atom contribute an unbonded electron in the pz orbital. The pz orbital of the neighboring atom is perpendicular to the plane to form a $\pi$ bond. The newly formed $\pi$ bond is half-filled. Studies have confirmed that the bond length between every two adjacent carbon atoms is $1.42 \mathrm{E}-10$ meters, and the angle between the bonds is $120^{\circ}$. In addition to the honeycomb layered structure where the $\sigma$ bond is linked to other carbon atoms to form a hexagonal ring, the pz orbital perpendicular to the layer plane of each carbon atom can form a large-atom large $\pi$ bond (similar to a benzene ring) through the entire layer, It has excellent electrical and optical properties. [34,35]

\section{Electronic effect}

The carrier mobility of graphene at room temperature is about $15000 \mathrm{~cm}^{2} /(\mathrm{V} \cdot \mathrm{s})$, which is more than 10 times that of silicon. Also it is more than twice of indium antimonide (InSb), which has the highest carrier mobility. Under certain conditions such as low temperature, the carrier mobility of graphene can even be as high as $250,000 \mathrm{~cm}^{2} /(\mathrm{V} \cdot \mathrm{s})$. Unlike many materials, the electron mobility of graphene is less affected by temperature changes because of its structure. At any temperature between 50 and $500 \mathrm{~K}$, the electron mobility of single-layer graphene is around $15000 \mathrm{~cm}^{2} /(\mathrm{V} \cdot \mathrm{s}) \cdot[36]$

In addition, the half-integer quantum Hall effect of electron carriers and hole carriers in graphene can be observed by changing the chemical potential through the action of an electric field, and scientists have observed this quantum Hall effect, which is similar to the Hall effect in bulk materials, of graphene at room temperature. The carriers in graphene follow a special quantum tunneling effect, which does not produce backscattering when they encounter impurities. This is due to the high carrier mobility of graphene. [37,38] Graphene is a zero-distance semiconductor because its conduction and valence band meet at Dirac point. The Brillouin edge of the momentum space at six positions at the Dirac point is divided into two groups of three equivalents. 
In contrast, the main point of traditional semiconductors is usually $\Gamma$, and the momentum is zero. [39]

Thermal performance

Graphene has very good thermal conductivity. The thermal conductivity of pure defect-free single-layer graphene is as high as $5300 \mathrm{~W} / \mathrm{mK}$, which is the carbon material with the highest thermal conductivity by far, higher than single-wall carbon nanotubes (3500W/mK) and multi-wall carbon nanotubes (3000W/mK). When it is used as a carrier, the thermal conductivity can also reach $600 \mathrm{~W} / \mathrm{mK}$. In addition, graphene's ballistic thermal conductivity can lower the lower limit of the ballistic thermal conductivity of carbon nanotubes per unit circumference and length. [40-42]

\section{Optical characteristics}

Graphene has very good optical properties, its absorption rate is about $2.3 \%$ in a wide wavelength range, and it looks almost transparent. Within the thickness range of several layers of graphene, each increase in thickness increases the absorption rate by $2.3 \%$. Large-area graphene films also have excellent optical characteristics, and their optical characteristics change with the change of graphene thickness. This is an unusual low-energy electronic structure possessed by single-layer graphene. By applying a magnetic field, the optical response of graphene nanoribbons can be tuned to the terahertz range. $[43,44]$ When the intensity of the incident light exceeds a certain critical value, the absorption of graphene will reach saturation. These characteristics allow graphene to be used as a passive mode-locked laser. This unique absorption may become saturated when the input light intensity exceeds a threshold, which is called the saturation effect. Graphene can be saturated easily under visible strong excitation in the near infrared region due to global optical absorption and zero band gap. Because of this special property, graphene is widely used in ultrafast photonics. The optical response of the 
graphene/graphene oxide layer can be tuned electrically. Under denser laser illumination, graphene may possess an optical nonlinear Kerr effect, which means the refractive change under an applied electric field, with a nonlinear phase shift.[45]

Graphene has excellent optical, electrical, and mechanical properties. It has important application prospects in materials science, micro-nano processing, energy, biomedicine, and drug delivery. It is considered to be a revolutionary material in the future. Andre Gemm and Konstantin Novoselov from the University of Manchester in UK successfully separated graphene from graphite by micromechanical stripping, and therefore jointly won the 2010 Nobel Prize in Physics. The common powder production methods of graphene are mechanical peeling method, redox method, and SiC epitaxial growth method, and the thin film production method is chemical vapor deposition (CVD).

\subsubsection{Mass production of graphene}

\section{Mechanical stripping}

The mechanical peeling method is a method for obtaining a thin layer of graphene material by using friction and relative motion between an object and graphene. This method is simple to operate, and the resulting graphene usually maintains a complete crystal structure. In 2004, two British sciences used transparent tape to peel off natural graphite layer by layer to obtain graphene, which was also classified as a mechanical peeling method. This method was once considered to have low production efficiency and could not be massproduced industrially. This method can produce micron-sized graphene, but its controllability is low, and it is difficult to achieve large-scale synthesis. 


\section{Redox}

The redox method is to oxidize natural graphite by using chemical reagents such as sulfuric acid, nitric acid, potassium permanganate, hydrogen peroxide, etc., to increase the spacing between graphite layers, and insert oxide between the graphite layers to produce graphite oxide.. Then, the reactant is washed with water, and the washed solid is dried at a low temperature to prepare graphite oxide powder. The graphite oxide powder is peeled off by methods such as physical peeling and high-temperature expansion to prepare graphene oxide. Finally, graphene oxide is reduced by chemical method to obtain graphene (RGO). This method is simple to operate and has a high output, but the product quality is low. The oxidation-reduction method uses strong acids such as sulfuric acid and nitric acid, which have a greater risk, and a large amount of water must be used for cleaning, which causes large environmental pollution.

\section{Epitaxial method}

The orientation epigenetic method is to use the growth matrix atomic structure to "seeding" graphene, first let carbon atoms infiltrate ruthenium at $1150{ }^{\circ} \mathrm{C}$, and then cool, after cooling to $850{ }^{\circ} \mathrm{C}$, a large number of carbon atoms absorbed before will float to the surface of ruthenium, The final lens-shaped single layer of carbon atoms will grow into a complete layer of graphene. After the first layer is covered, the second layer begins to grow. The bottom graphene will have a strong interaction with ruthenium, and after the second layer, it will be almost completely separated from ruthenium, leaving only weak electrical coupling. However, the thickness of graphene sheets produced by this method is often uneven, and the adhesion between graphene and the matrix will affect the characteristics of the carbon layer.

\section{Silicon carbide epitaxy}

In the SiC epitaxy method, silicon atoms are sublimated out of the material 
under ultra-high vacuum and high temperature environment, and the remaining $\mathrm{C}$ atoms are reconstructed by self-assembly to obtain graphene based on SiC substrate. This method can obtain high-quality graphene, but this method requires higher equipment.

\subsubsection{Bilayer graphene}

Bilayer graphene is a material consisting of two layers of graphene. One of the first reports of bilayer graphene was in the seminal 2004 Science paper by Geim and colleagues in which they described devices "which contained just one, two, or three atomic layer structure"[46,47]

Bilayer graphene can exist in the form of an $A B$ or Bernal stack, where half of the atoms are directly above the center of the hexagon in the lower graphene sheet, and the other half of the atoms are on the atoms, or less commonly, in the form of AA Form, the layers are perfectly aligned. In Bernal (Bernal) stacked graphene, double boundaries are common. Transition from $A B$ stack to $B A$ stack. The twisted layer rotating relative to another layer has also been extensively studied.[48,49]

\section{Superconductivity of twisted bilayer graphene}

The time goes back to 2011. Although it was realized at that time that two layers of graphene were stacked at a certain angle, a two-dimensional molar superlattice could be formed and new physical phenomena would be brought about. However, it was not until the theoretical physicists Professor Allan $\mathrm{H}$. MacDonald and Dr. Rafi Bistritzer in the United States calculated that the velocity of electrons in the double-layer graphene superlattice with a rotation angle of $1.1^{\circ}$ would be greatly reduced. The layered graphene superlattice 
contains rich multi-body strong correlation physics. In 2018, Yuan Cao and his supervisor Professor Pablo Jarillo-Herrero from the Massachusetts Institute of Technology and colleagues of Harvard University and the National Institute of Materials Science in Tsukuba, Japan reported the discovery of superconductivity in two layers of graphene. The twist angle between the two layers of graphene is $1.1^{\circ}$. The discovery was announced in Nature in March 2018. The bilayer graphene sample was prepared by peeling to get a single layer of graphene, and the second layer is manually rotated to a set angle relative to the first layer. When the temperature of samples reached $1.7 \mathrm{~K}$, the transition of the sample is transformed from the metallic state to the insulating state. It is also discovered that if a certain number of electrons are added to the transformed insulation state, superconductivity can be induced.[50,51]

\subsubsection{Application of graphene}

With the gradual breakthrough of mass production and large-scale problems, the industrial application of graphene is accelerating. Based on the existing research results, the first field to achieve commercial application may be mobile equipment, aerospace, and new energy Battery field.

\section{Transistor}

Graphene can be used to make transistors. Due to the high stability of the graphene structure, this transistor can still work steadily on a scale close to a single atom. In contrast, the current transistors made of silicon lose stability at a scale of about 10 nanometers; the characteristic that the electrons in graphene react to the external field is extremely fast, and the transistors made of it can achieve Very high operating frequency. For example, IBM Corporation announced in February 2010 that the operating frequency of graphene 
transistors has been increased to $100 \mathrm{GHz}$, exceeding that of silicon transistors of the same size.

New energy battery

New energy batteries are also an important area for the first commercial use of graphene. Massachusetts Institute of Technology has successfully developed a flexible photovoltaic panel with a graphene nano-coating on the surface, which can greatly reduce the cost of manufacturing transparent and deformable solar cells. Such batteries may be used in night vision goggles, cameras and other small digital Application in the device. In addition, the successful development of graphene super batteries has also solved the problems of insufficient capacity and long charging time of new energy vehicle batteries, which has greatly accelerated the development of the new energy battery industry.

\subsection{III-V compound semiconductor}

Compound semiconductor is a type of semiconductor material that is composed of compounds. Typically, compound semiconductors are composed of two or more elements. Common elements in compound semiconductors are from Group IIIA boron, aluminum, gallium, indium, thallium and group VA elements nitrogen, phosphorus, arsenic, antimony and bismuth. Generally speaking, the III-V semiconductor is a binary compound composed of the above-mentioned group IIIA and group VA elements, and the chemical ratio of their components is $1: 1$. It includes the following compounds: BN, BP, BAs, AIN, AIP, AIAs, AISb, GaN, GaP, GaAs, GaSb, InN, InP, InAs, InSb.[52-54] 
The following figures are simulation result for typical III-V compound semiconductor. All the results are simulated by quantumATK toolkit. The results are mainly for showing the bandstructure and projected density of state(PDOS) . The bandgap value may not be quite accuracy due to the lack of proper correction in simulation. In order to get proper correction in simulation, there must be specific calculation and comparison between different methods, and this is quite large amount of work but I don't have enough time to finish. So the results here focus on the shape and potential of the bandstructure but not the accuracy value.

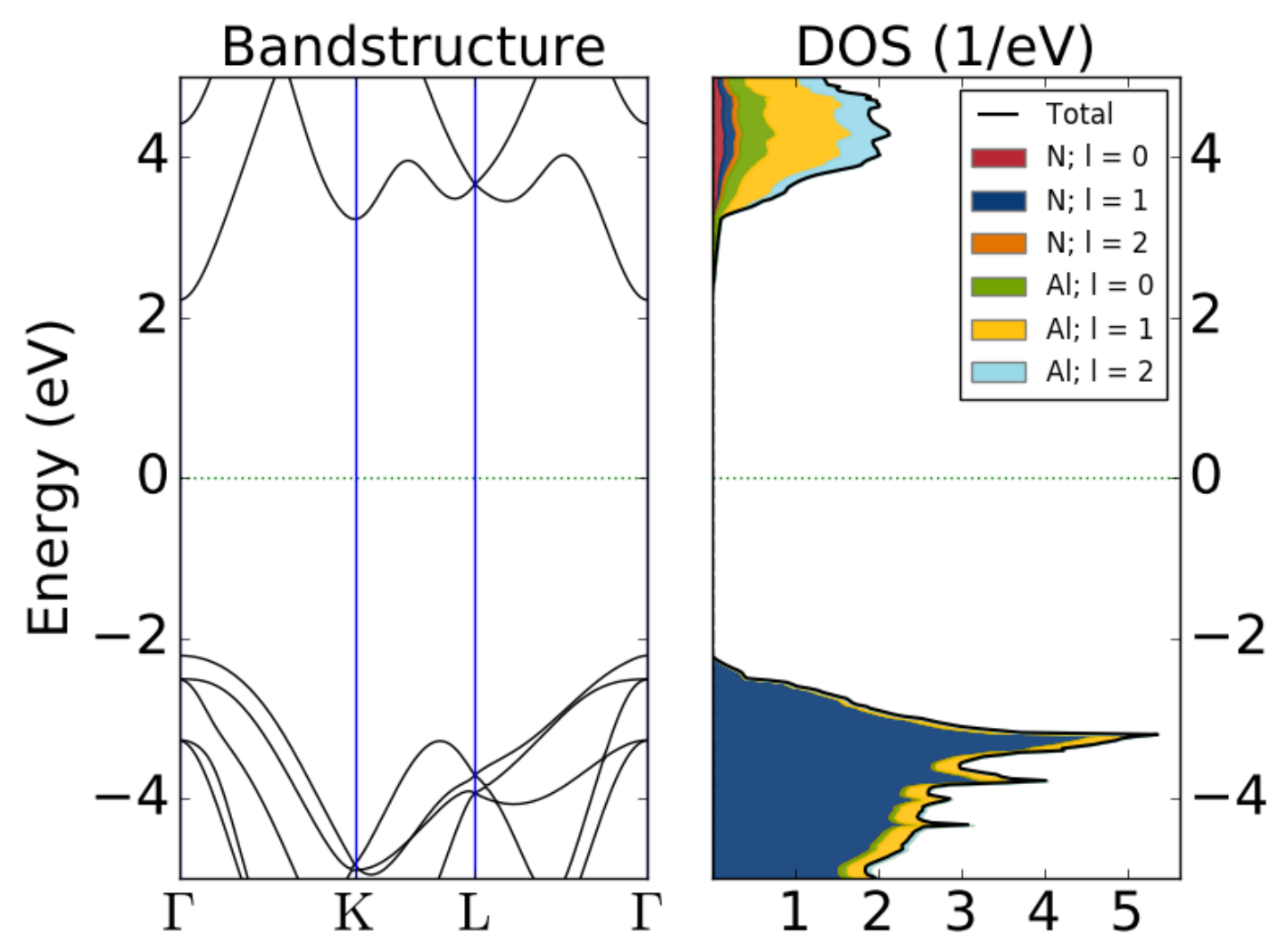

Figure 1.1 the bandstructure and PDOS of AIAs 

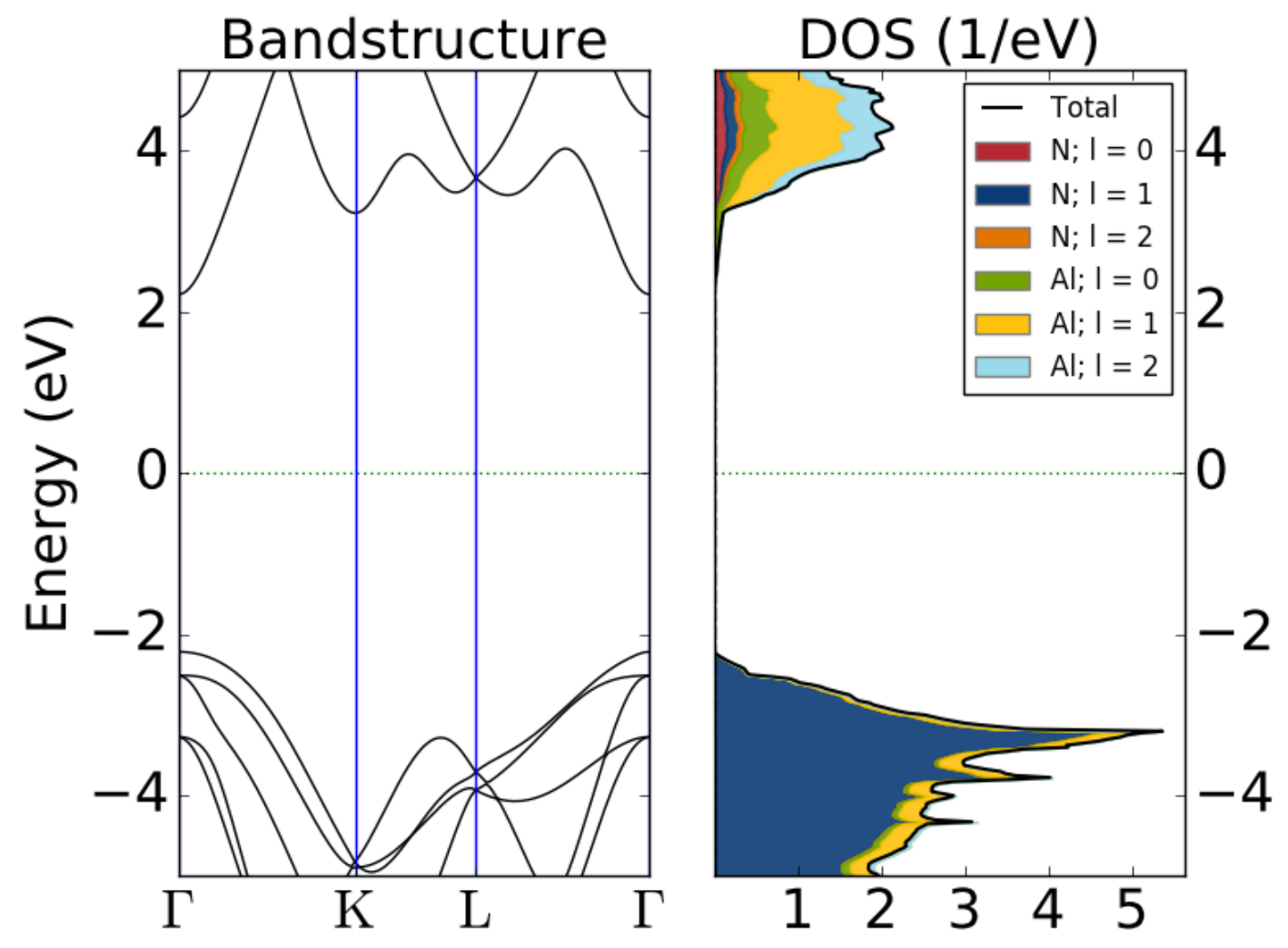

Figure 1.2 the bandstructure and PDOS of AIN

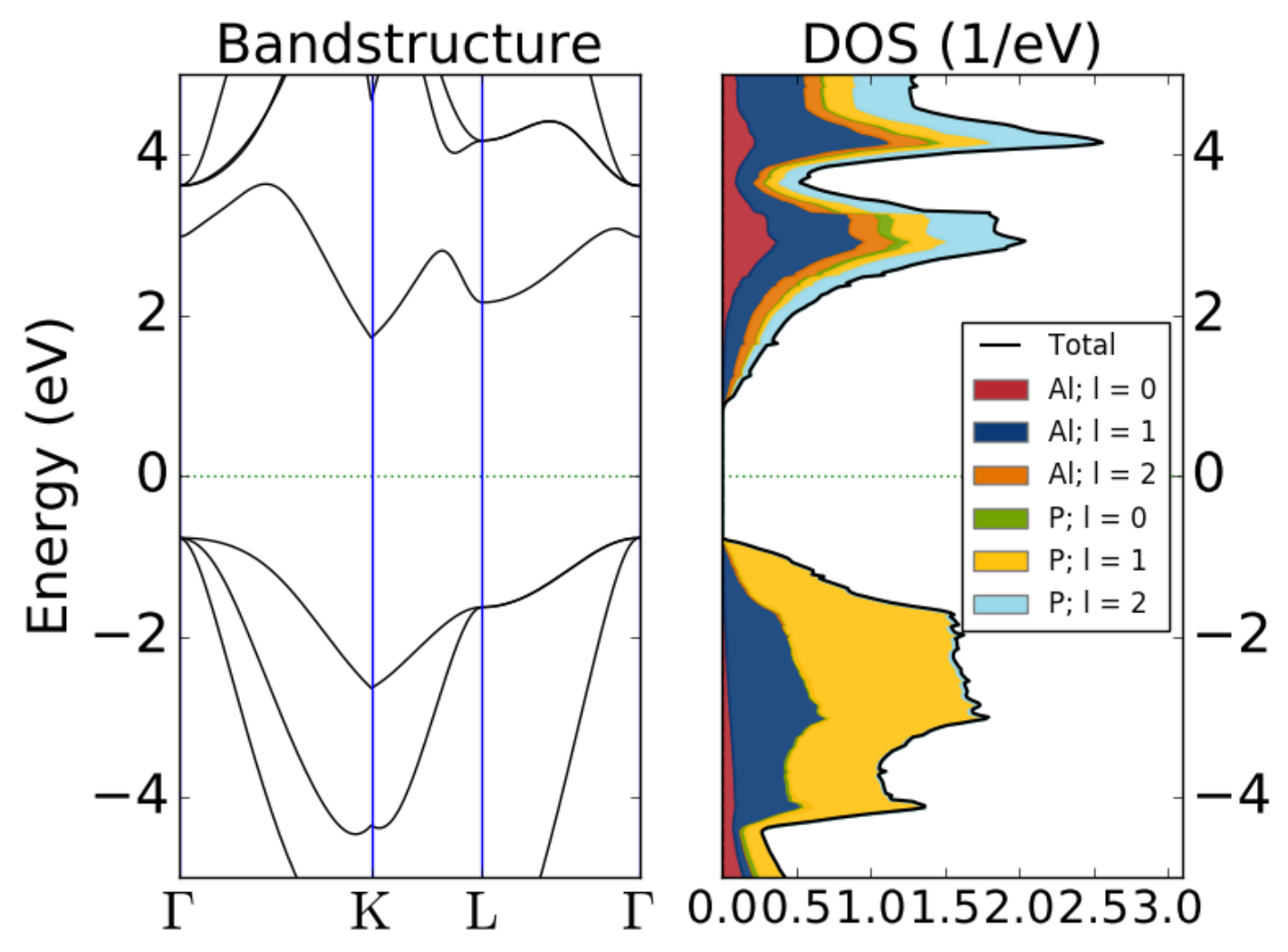

Figure 1.3 the bandstructure and PDOS of AIP 

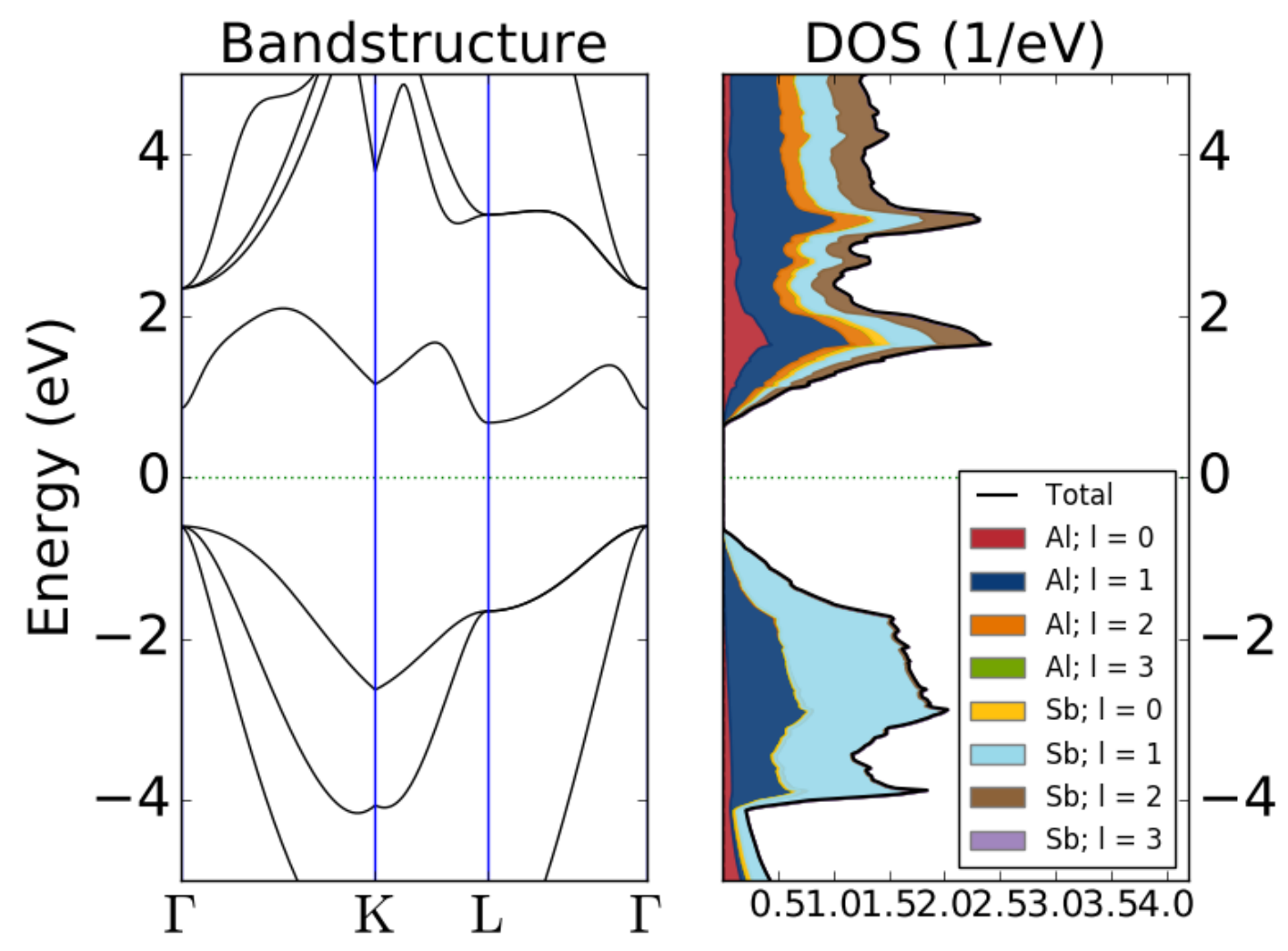

Figure 1.4 the bandstructure and PDOS of AISb
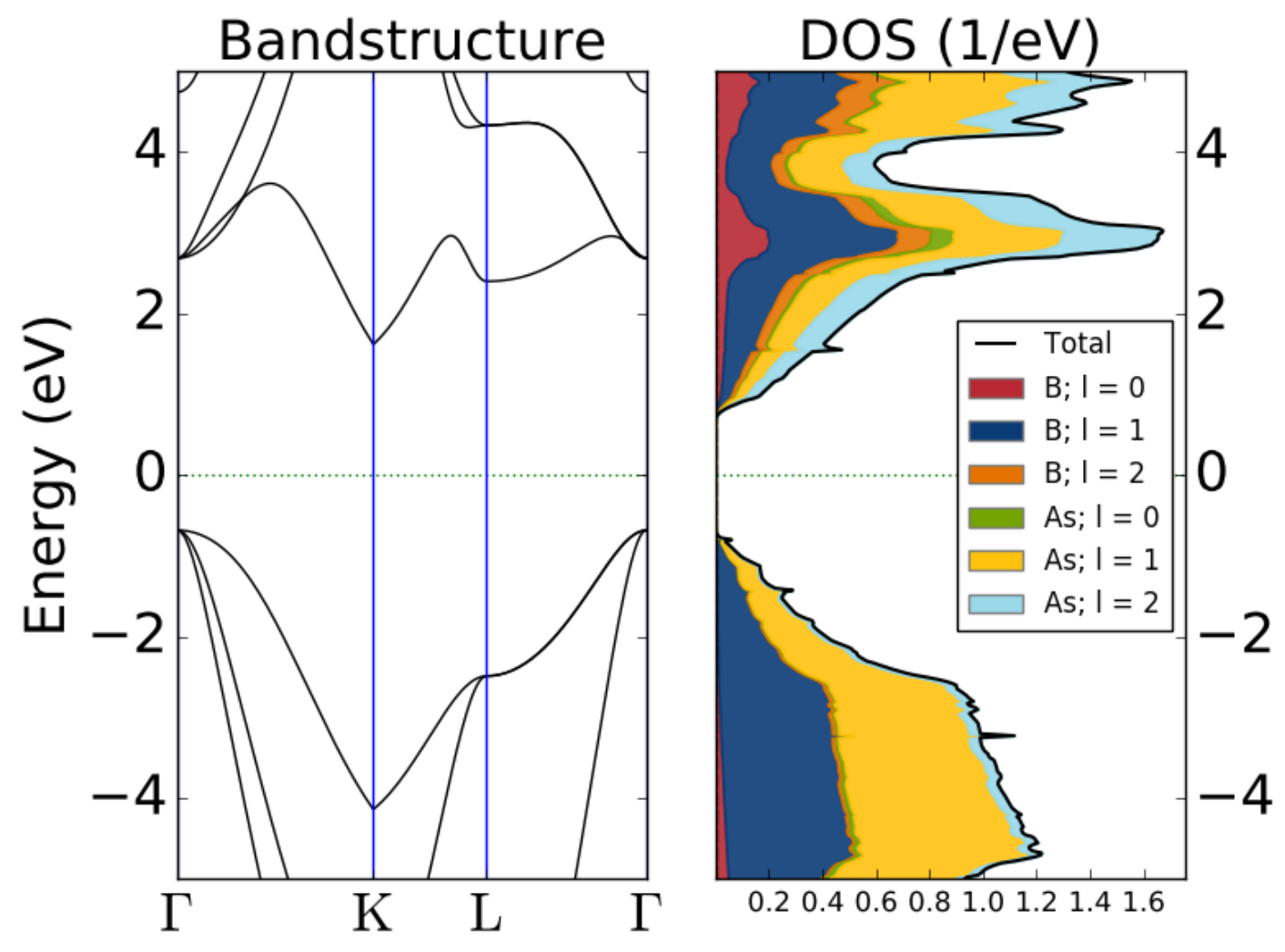

Figure 1.5 the bandstructure and PDOS of BAs 


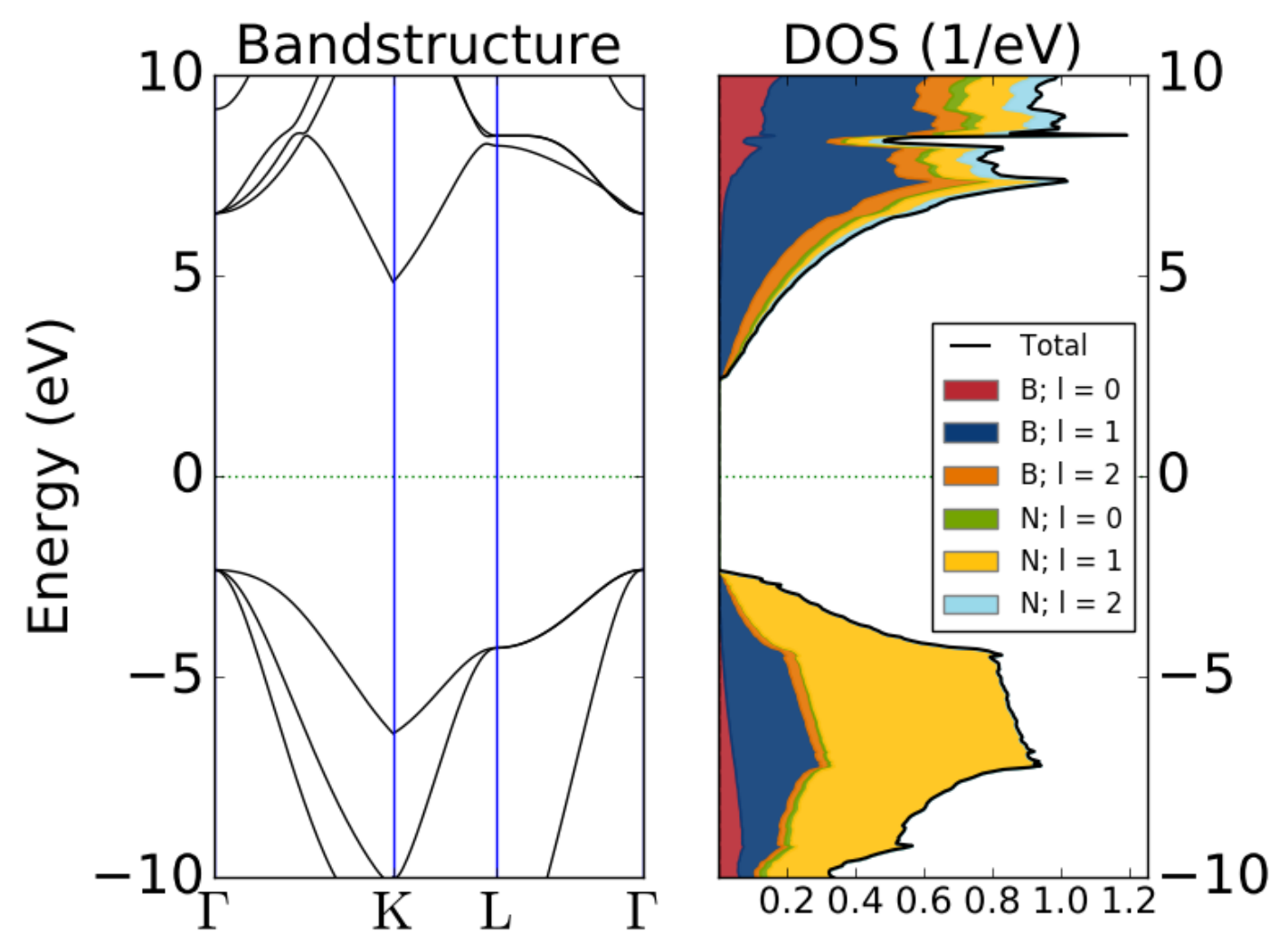

Figure 1.6 the bandstructure and PDOS of $B N$ 

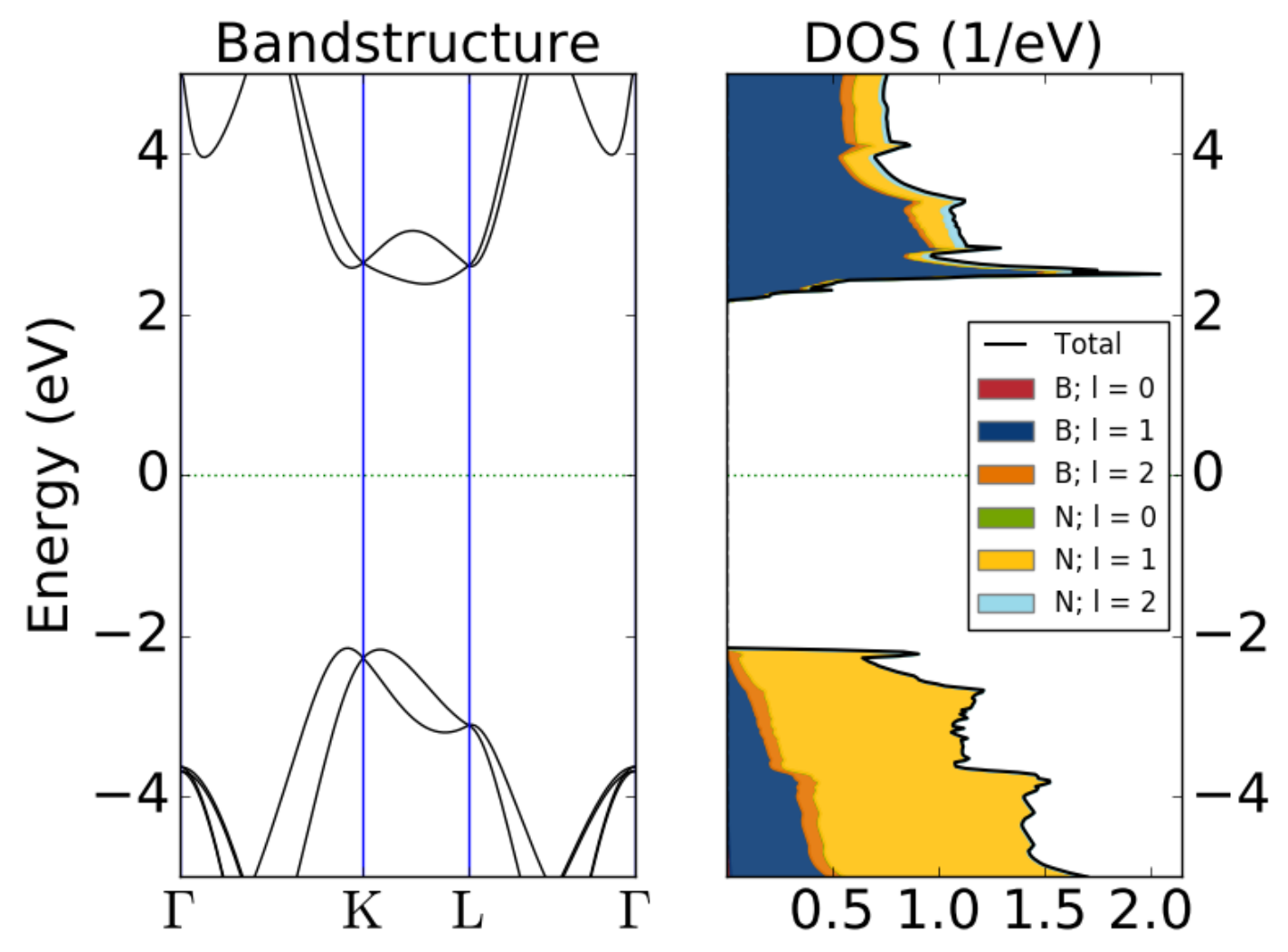

Figure 1.7 the bandstructure and PDOS of BN(hexagonal)
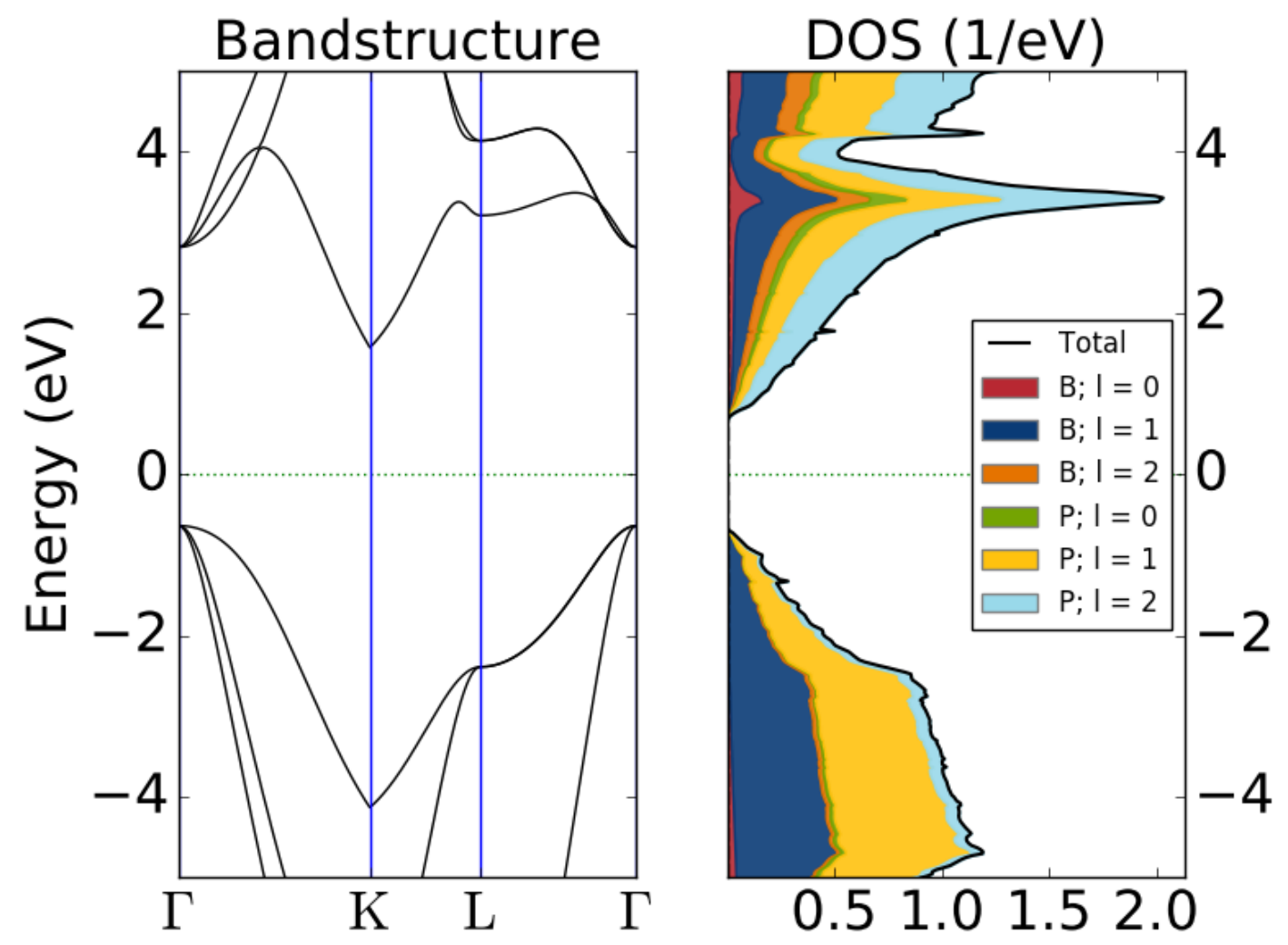

Figure 1.8 the bandstructure and PDOS of BP 

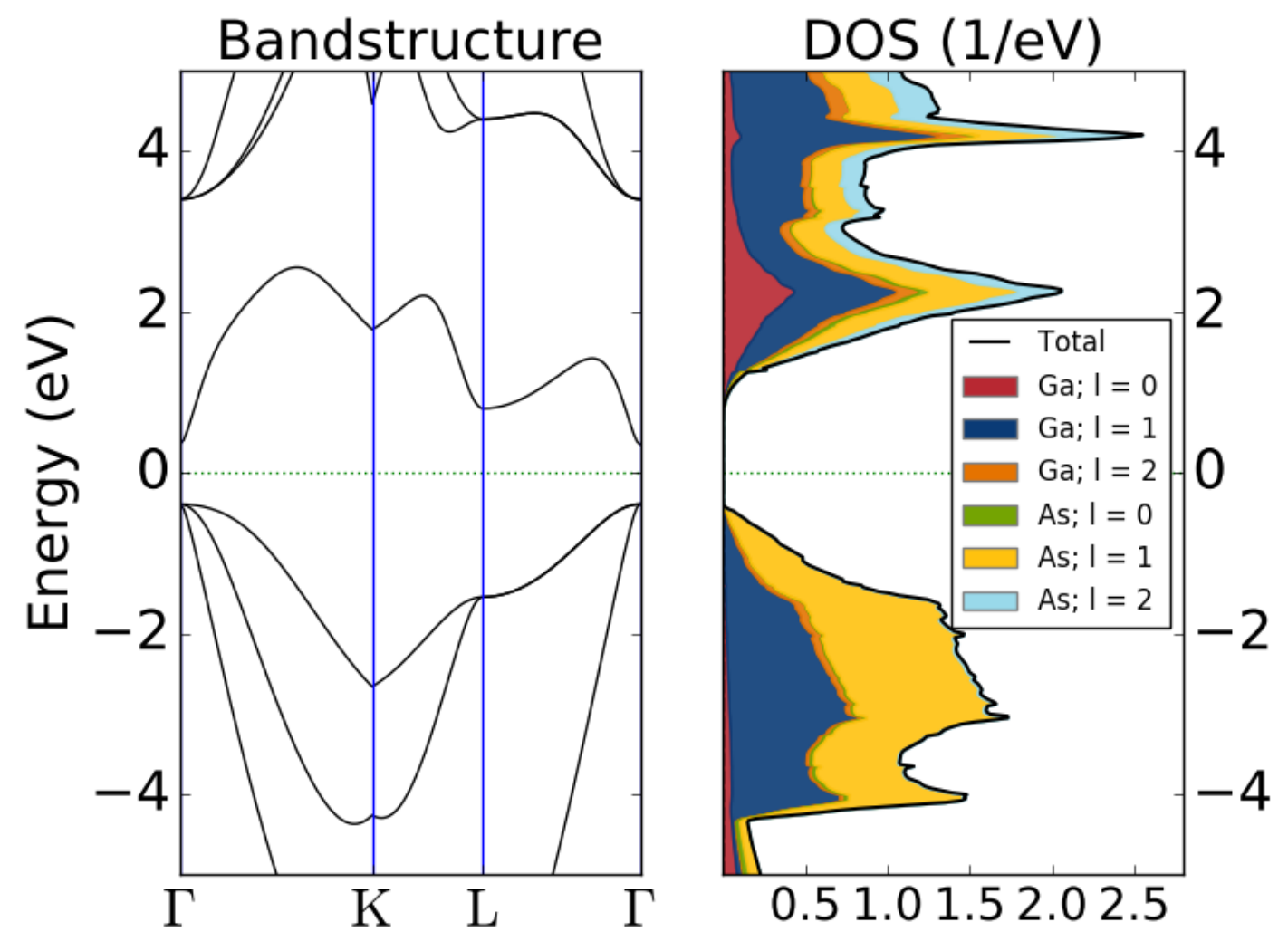

Figure 1.9 the bandstructure and PDOS of GaAs 

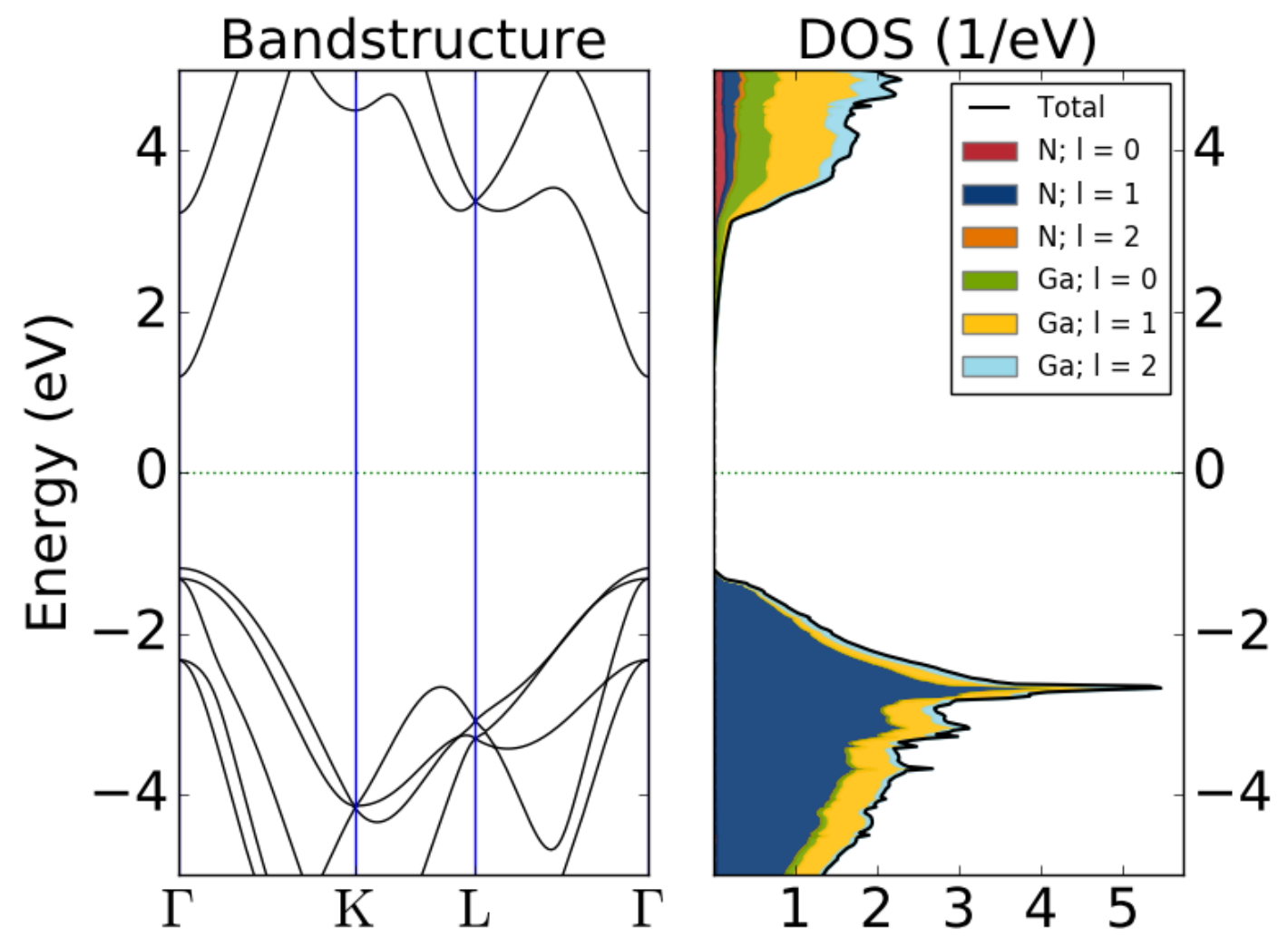

Figure 1.10 the bandstructure and PDOS of GaN
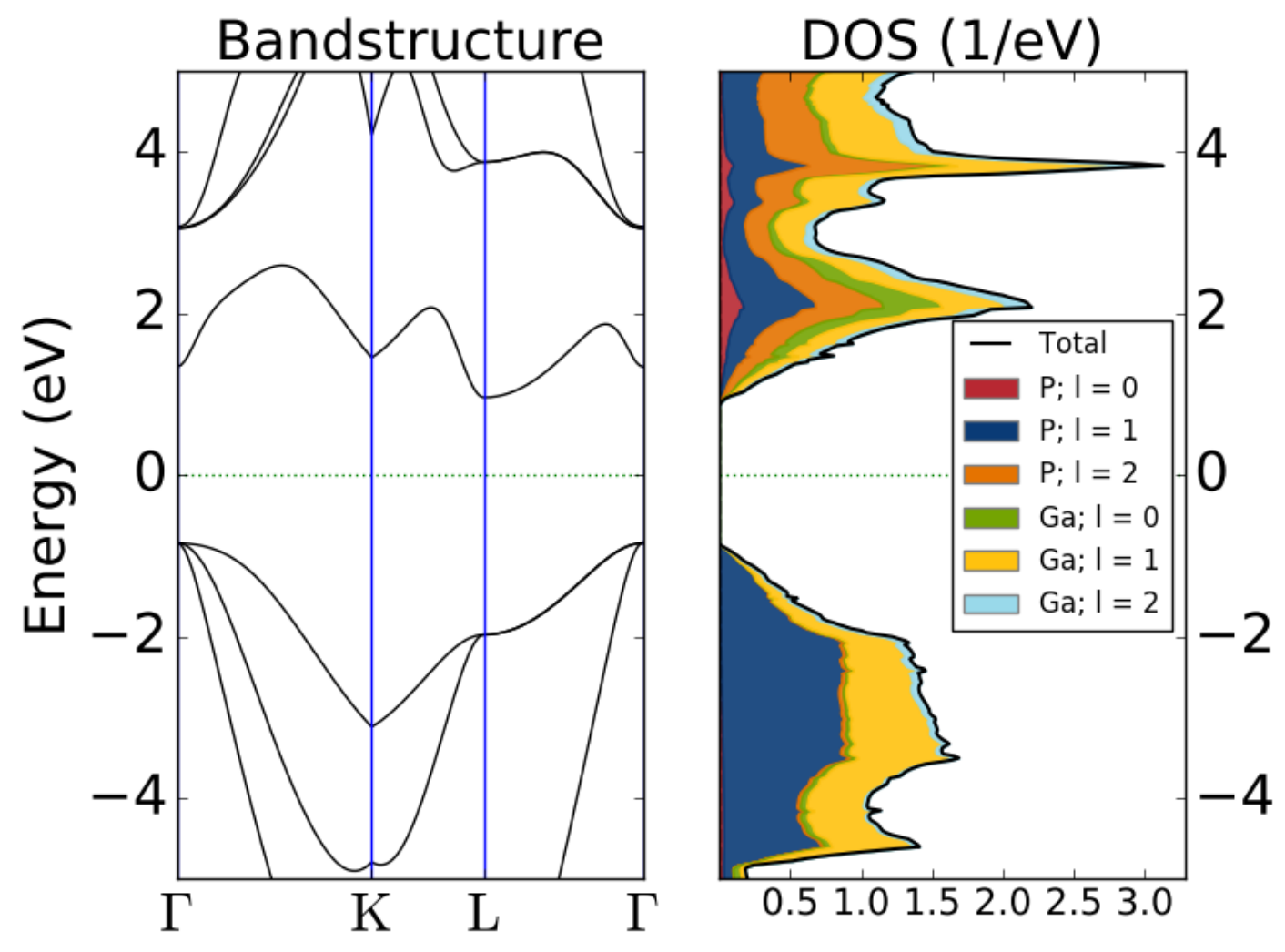

Figure 1.11 the bandstructure and PDOS of GaP 


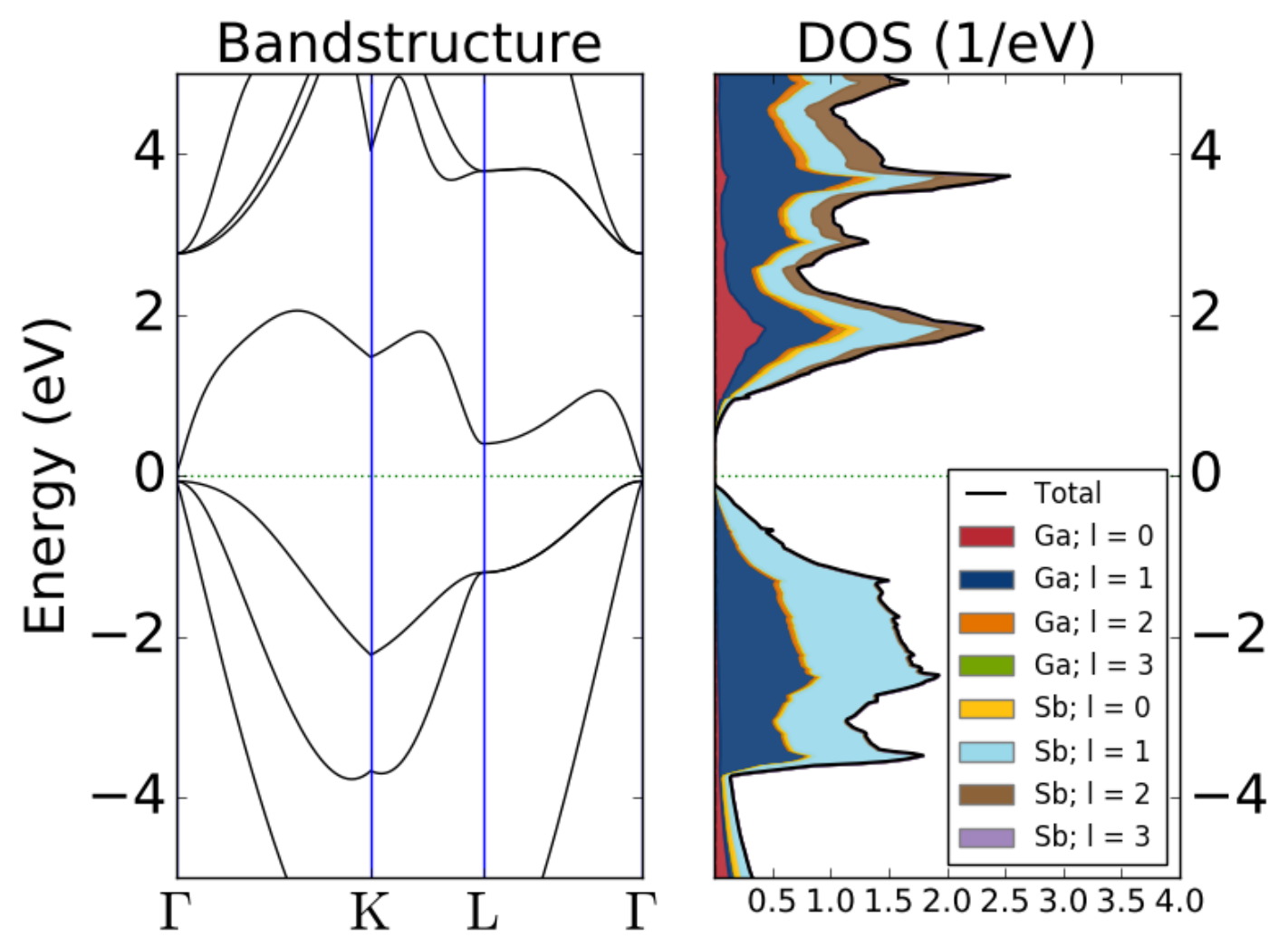

Figure 1.12 the bandstructure and PDOS of GaSb 

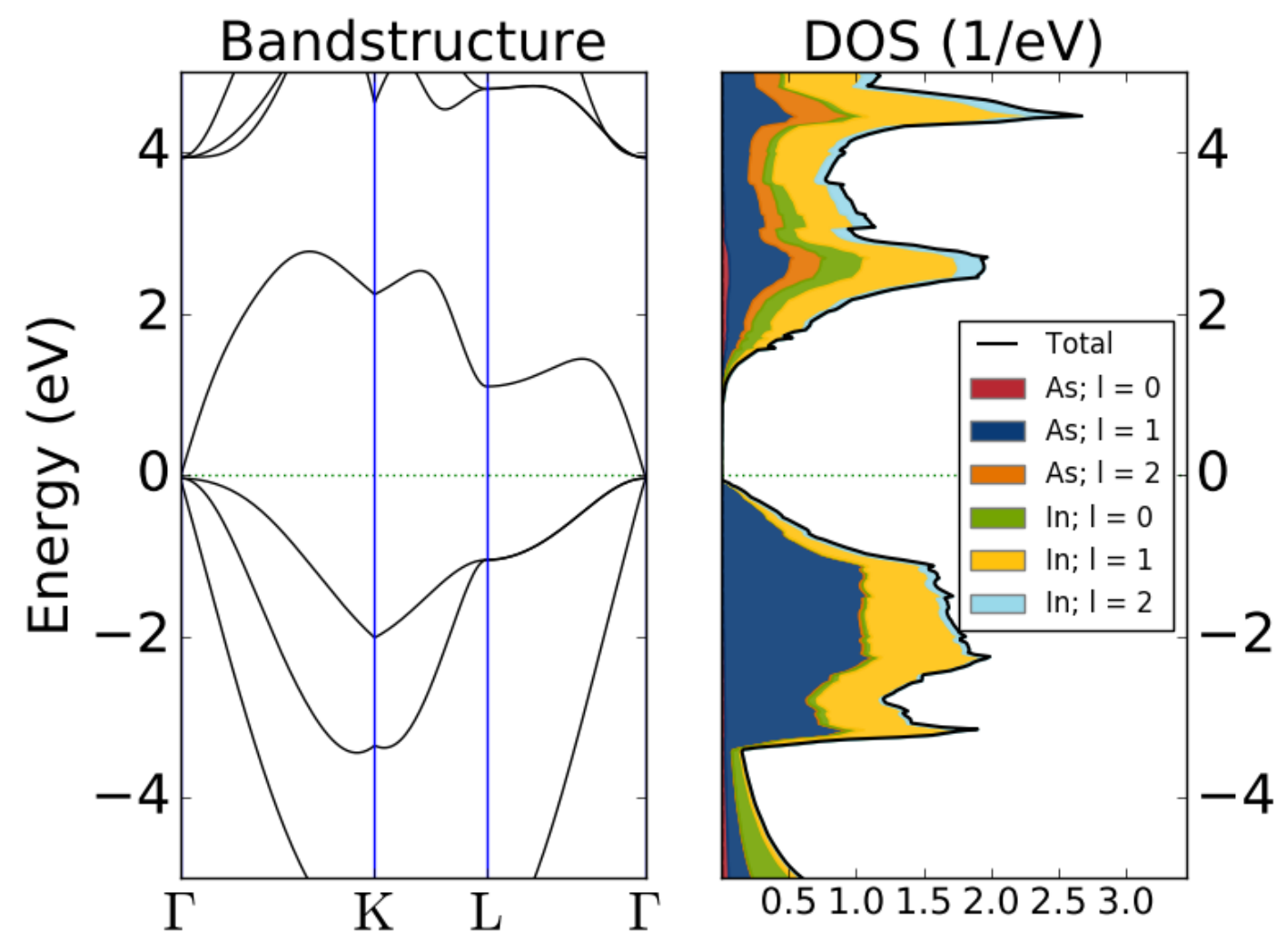

Figure 1.13 the bandstructure and PDOS of InAs
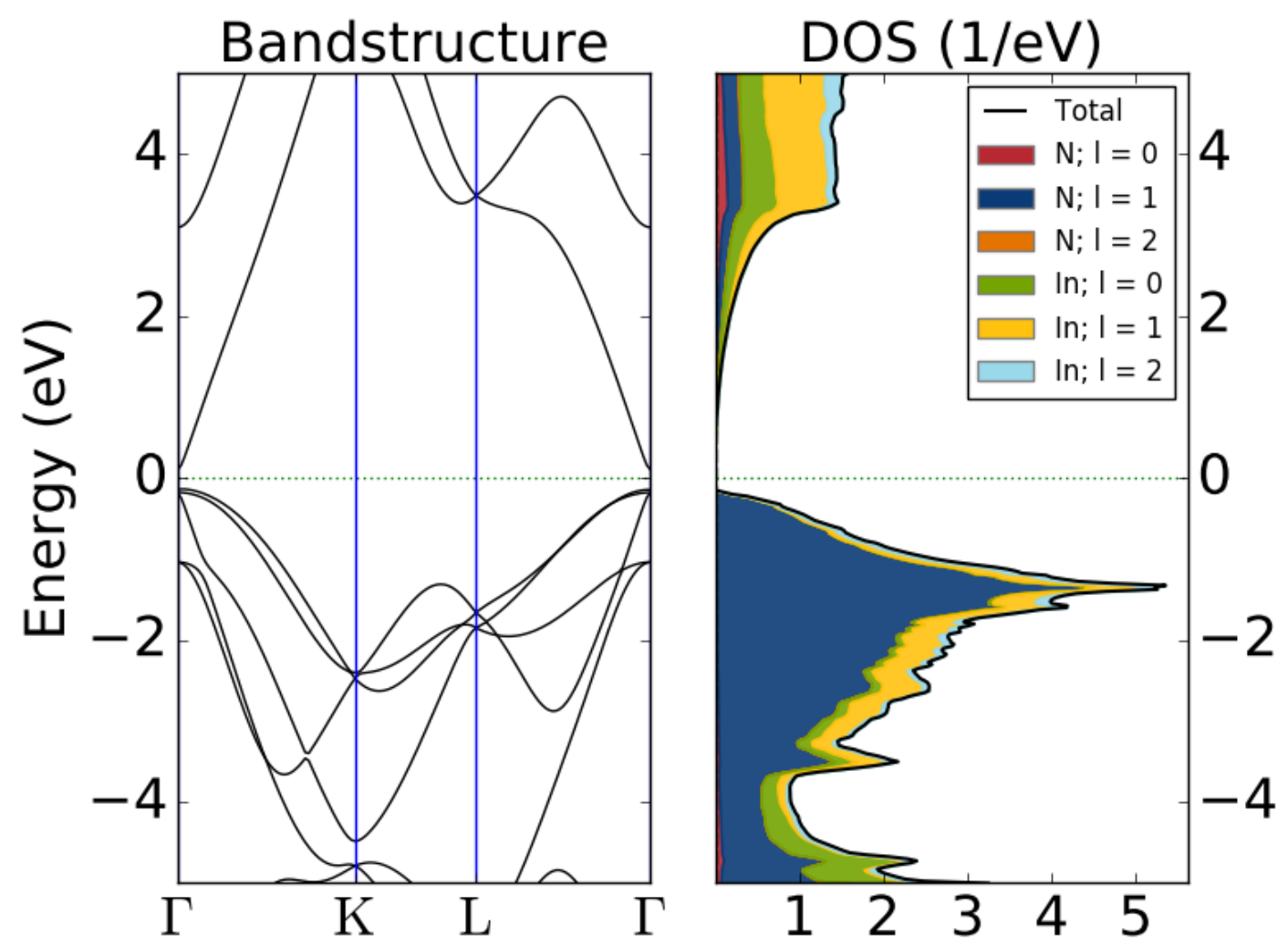

Figure 1.14 the bandstructure and PDOS of InN 

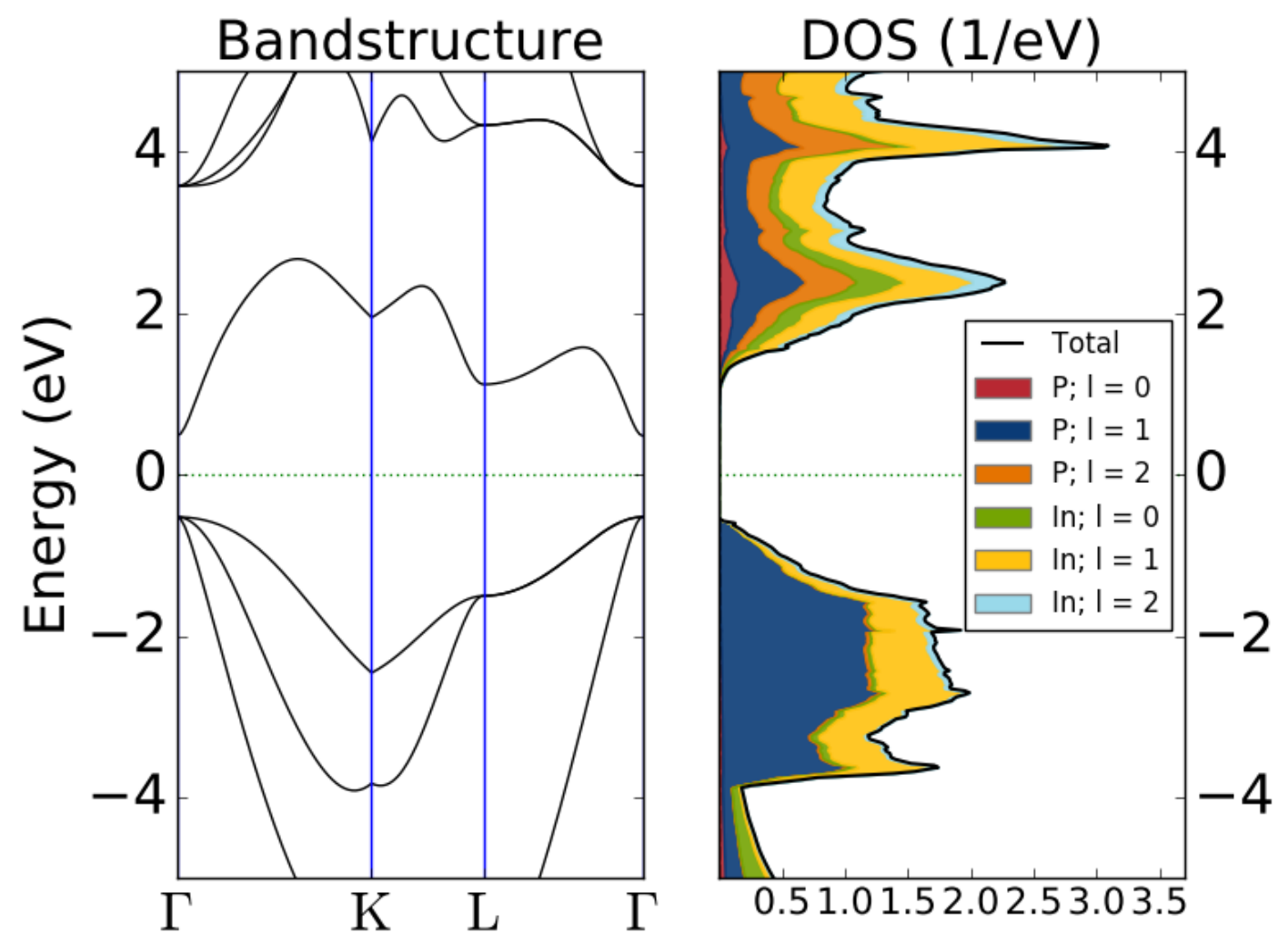

Figure 1.15 the bandstructure and PDOS of InP
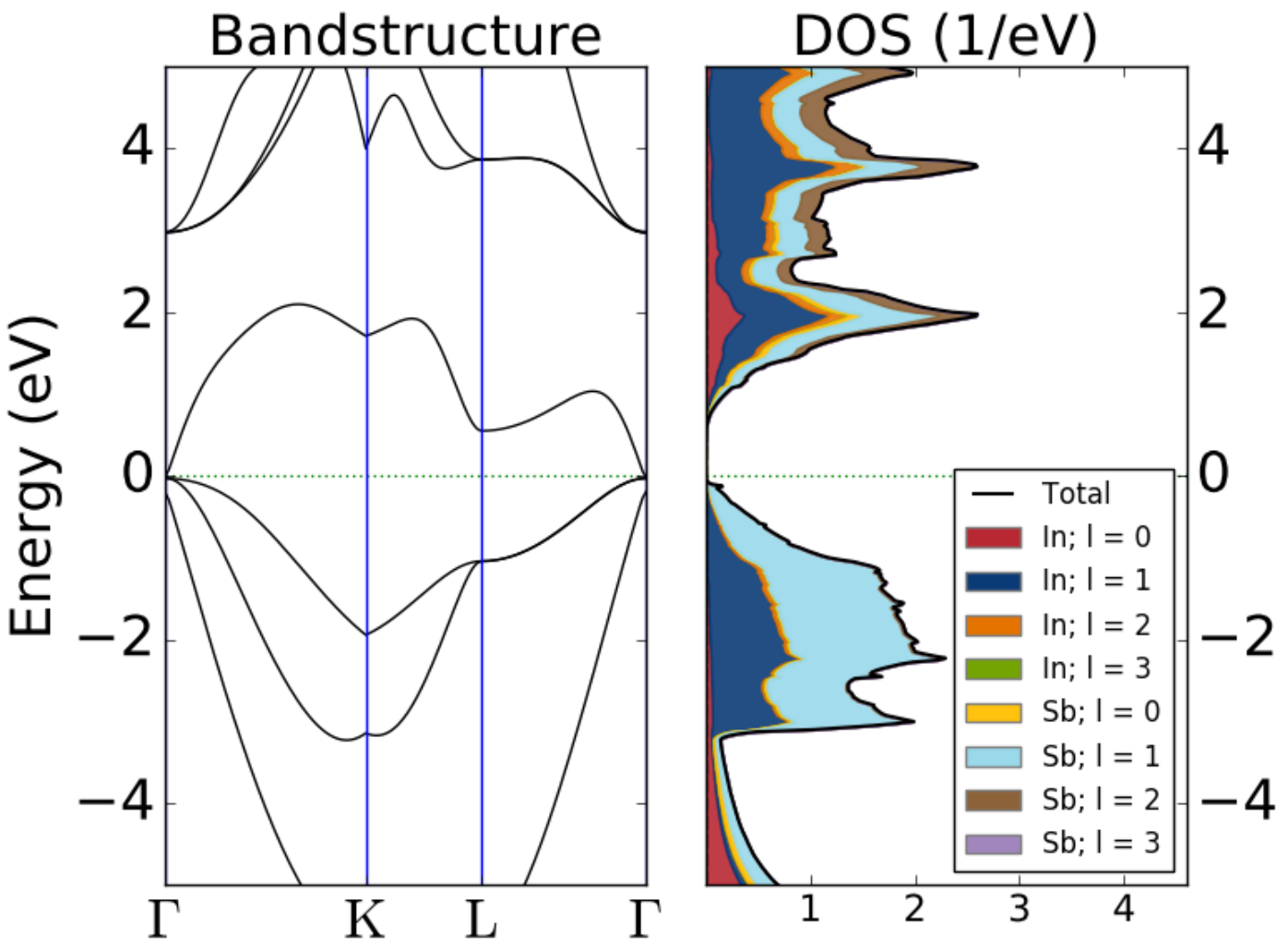

Figure 1.16 the bandstructure and PDOS of InSb 
Table 1.1 Bandgap and Bandstructure type

\begin{tabular}{|c|c|c|c|}
\hline Formula & Direct & Indirect & Type \\
\hline & Bandgap(eV) & Bandgap(eV) & \\
\hline AlAs & 2.45 & 2.32 & Indirect \\
\hline AIN & 4.43 & 4.43 & Direct \\
\hline AIP & 3.74 & 2.49 & Indirect \\
\hline AlSb & 1.45 & 1.28 & Direct \\
\hline BAs & 3.35 & 2.29 & Indirect \\
\hline BN & 8.87 & 7.16 & Indirect \\
\hline BNhex & 4.67 & 4.53 & Direct \\
\hline BP & 3.45 & 2.20 & Indirect \\
\hline GaAs & 0.76 & 0.76 & Direct \\
\hline GaN & 2.37 & 2.37 & Direct \\
\hline GaP & 2.17 & 1.79 & Indirect \\
\hline GaSb & 0.10 & 0.10 & Direct \\
\hline InAs & 0.00 & 0.00 & Direct \\
\hline $\ln N$ & 0.25 & 0.24 & Indirect \\
\hline $\ln P$ & 1.00 & 1.00 & Direct \\
\hline InSb & 0.00 & 0.00 & Direct \\
\hline
\end{tabular}




\subsection{Gallium Nitride(GaN)}

GaN is one of typical III-V compound semiconductor materials. This compound has a structure similar to wurtzite and has a high hardness. Gallium nitride has a wide energy gap of 3.4 electron volts and can be used in high-power, highspeed optoelectronic components. It has been applied in light-emitting diodes since 1990, mainly used in violet laser diodes. [55]

Like other group III element nitrides, gallium nitride is less sensitive to ionizing radiation, which makes it suitable for solar cell arrays for satellites. Military and space applications may also benefit, because GaN devices show stability in radiated environments. Compared to gallium arsenide (GaAs) transistors, gallium nitride transistors can operate at much higher temperatures and voltages, so they are ideal microwave frequency power amplifiers[56]

\section{LEDs}

The GaN material is an ideal short-wavelength light-emitting device material, and the band gap of GaN and its alloy covers the spectral range from red to ultraviolet.[57]

\section{Transistors}

The GaN material has a low heat generation rate and a high breakdown electric field and is an important material for developing high-temperature high-power electronic devices and high-frequency microwave devices. [58] At present, with the progress of MBE technology in the application of GaN materials and the breakthrough of key thin film growth technologies, a variety of GaN heterostructures have been successfully grown. New devices such as metal field effect transistors (MESFETs), heterojunction field effect transistors (HFETs), modulation doped field effect transistors (MODFETs), etc. were 
prepared using GaN materials. Modulated and doped AIGaN/GaN structure has high electron mobility $\left(2000 \mathrm{~cm}^{2} / \mathrm{v} \cdot \mathrm{s}\right)$, high saturation speed $\left(1 \times 10^{7} \mathrm{~cm} / \mathrm{s}\right)$, and low dielectric constant, and is the preferred material for making microwave devices; $[59,60]$ GaN The wider forbidden band width $(3.4 \mathrm{eV})$ and sapphire as the substrate have good heat dissipation performance, which is conducive to the device working under high power conditions.

\section{Microwave device}

GaN high-frequency high-power microwave devices have begun to be used in military radar, smart weapons and communication systems. In the future, GaN microwave devices are expected to be used in civilian fields such as 4G-5G mobile communication base stations.[61]

\section{Lasers and detectors}

In the field of laser and detector applications, GaN lasers have been successfully used in Blu-ray. Because of its excellent photoelectric properties and radiation resistance, gallium nitride can also be used as a high-energy ray detector. GaN-based UV detectors can be used in missile early warning, satellite secret communications, various environmental monitoring, chemical biological detection and other fields, such as nuclear radiation detectors, X-ray imagers, etc. [57] 


\subsection{Reference List}

[1] Levine, Ira N. (1991). Quantum Chemistry. Englewood Cliffs, New jersey: Prentice Hall. pp. $455-544$.

[2] Parr, Robert G.; Craig D. P.; Ross, I. G (1950). "Molecular Orbital Calculations of the Lower Excited Electronic Levels of Benzene, Configuration Interaction included". Journal of Chemical Physics. 18 (12): 1561-1563.

[3] Parr, R. G. (1990). "On the genesis of a theory". Int. J. Quantum Chem. 37 (4): 327-347.

[4] Leach, Dr Andrew (2001-01-30). Molecular Modelling: Principles and Applications (2 ed.). Harlow: Prentice Hall.

[5] Friesner, Richard A. (2005-05-10). "Ab initio quantum chemistry: Methodology and applications". Proceedings of the National Academy of Sciences of the United States of America. 102 (19): 6648-6653.

[6] Cramer, Christopher J. (2002). Essentials of Computational Chemistry. Chichester: John Wiley \& Sons, Ltd. pp. 153-189.

[7] Cramer, Christopher J. (2002). Essentials of Computational Chemistry. Chichester: John Wiley \& Sons, Ltd. pp. 191-232.

[8] Jensen, Frank (2007). Introduction to Computational Chemistry. Chichester, England: John Wiley and Sons. pp. 98-149.

[9] Van Mourik, Tanja; Gdanitz, Robert J. (2002). "A critical note on density functional theory studies on rare-gas dimers". Journal of Chemical Physics. 116 (22): 9620-9623.

[10] Vondrášek, Jiří; Bendová, Lada; Klusák, Vojtěch; Hobza, Pavel (2005). "Unexpectedly strong energy stabilization inside the hydrophobic core of small protein rubredoxin mediated by aromatic residues: correlated ab initio quantum chemical calculations". Journal of the American Chemical Society. 127 (8): 2615-2619

[11] Grimme, Stefan (2006). "Semiempirical hybrid density functional with perturbative second-order correlation". Journal of Chemical Physics. 124(3): 034108. 
[12] Zimmerli, Urs; Parrinello, Michele; Koumoutsakos, Petros (2004). "Dispersion corrections to density functionals for water aromatic interactions". Journal of Chemical Physics. 120 (6): 2693-2699

[13] Grimme, Stefan (2004). "Accurate description of van der Waals complexes by density functional theory including empirical corrections". Journal of Computational Chemistry. 25 (12): 1463-1473.

[14] Von Lilienfeld, O. Anatole; Tavernelli, Ivano; Rothlisberger, Ursula; Sebastiani, Daniel (2004). "Optimization of effective atom centered potentials for London dispersion forces in density functional theory"(PDF). Physical Review Letters. 93 (15): 153004.

[15] Tkatchenko, Alexandre; Scheffler, Matthias (2009). "Accurate Molecular Van Der Waals Interactions from Ground-State Electron Density and Free-Atom Reference Data". Physical Review Letters. 102 (7): 073005.

[16] Medvedev, Michael G.; Bushmarinov, Ivan S.; Sun, Jianwei; Perdew, John P.; Lyssenko, Konstantin A. (2017-01-05). "Density functional theory is straying from the path toward the exact functional". Science. 355(6320): 49-52

[17] Nagy, Density functional theory and application to atoms and molecules, Rev. 298 (1998) $1-79$.

[18] Romaka, V.V. Romaka, N. Melnychenko, Yu. Stadnyk, L. Bohun, A. Horyn, Experimental and DFT study of the VeCoeSb ternary system[J] , Journal of Alloys and Compounds 739 (2018) 771-779

[19] Eugene S. Kryachko, Eduardo V. Ludena, Density functional theory: Foundations reviewed[J], Physics Reports 544 (2014) 123-239

[20] B. Obot, D.D. Macdonald, Z.M. Gasem, Density functional theory (DFT) as a powerful tool for designing neworganic corrosion inhibitors. Part 1: An overview [J], Corrosion Science $99(2015) 1-30$

[21] G.J. Snyder, E.S. Toberer, Complex thermoelectric materials, Nature Materials 7 (2008) 105-114.

[22] G.S. Nolas, J. Poon, M. Kanatzidis, Recent developments in bulk thermoelectric materials, MRS Bulletin 31 (2006) 199-205. 
[23] H. Bottner, G. Chen, R. Venkatasubramanian, Aspects of thinfilm superlattice thermoelectric materials, devices, and applications, MRS Bulletin 31 (2006) 211-217.

[24] T.M. Tritt, M.A. Subramanian, Thermoelectric materials, phenomena, and applications: a bird's eye view, MRS Bulletin 31 (2006) 188-194.

[25] L.E. Bell, Cooling, heating, generating power, and recovering waste heat with thermoelectric systems, Science (New York, NY) 321 (2008) 1457-1461.

[26] A.J. Minnich, M.S. Dresselhaus, Z.F. Ren, G. Chen, Bulk nanostructured thermoelectric materials: current research and future prospects, Energy \& Environmental Science 2 (2009) 466-479.

[27] Y.C. Lan, A.J. Minnich, G. Chen, Z.F. Ren, Enhancement of thermoelectric figure-of-merit by a bulk nanostructuring approach, Advanced Functional Materials 20 (2010) 357-376. [28] J. Baxter, Z.X. Bian, G. Chen, D. Danielson, M.S. Dresselhaus, A.G. Fedorov, et al., Nanoscale design to enable the revolution in renewable energy, Energy \& Environmental Science 2 (2009) 559-588.

[29] H.J. Goldsmid, R.W. Douglas, The use of semiconductors in thermoelectric refrigeration, British Journal of Applied Physics 5 (1954) 386-390.

[30] Ozdemir, Burak; Barone, Veronica (2020). "Thickness dependence of solar cell efficiency in transition metal dichalcogenides MX2 (M: Mo, W; X: S, Se, Te)". Solar Energy Materials and Solar Cells. 212: 110557.

[31] Garcia, J. C.; de Lima, D. B.; Assali, L. V. C.; Justo, J. F. (2011). "Group IV grapheneand graphane-like nanosheets". J. Phys. Chem. C. 115 (27)

[32] Xu, Yang; Cheng, Cheng; Du, Sichao; Yang, Jianyi; Yu, Bin; Luo, Jack; Yin, Wenyan; Li, Erping; Dong, Shurong; Ye, Peide; Duan, Xiangfeng (2016). "Contacts between Two- and Three-Dimensional Materials: Ohmic, Schottky, and p-n Heterojunctions". ACS Nano. 10 (5): 4895-4919.

[33] Novoselov, K. S.; Geim, A. K.; Morozov, S. V.; Jiang, D.; Zhang, Y.; Dubonos, S. V.; Grigorieva, I. V.; Firsov, A. A. (22 October 2004). "Electric Field Effect in Atomically Thin Carbon Films". Science. 306 (5696): 666-669. 
[34] Bonaccorso, F.; Colombo, L.; Yu, G.; Stoller, M.; Tozzini, V.; Ferrari, A. C.; Ruoff, R. S.; Pellegrini, V. (2015). "Graphene, related two-dimensional crystals, and hybrid systems for energy conversion and storage". Science. 347 (6217): 1246501.

[35] Cooper, Daniel R.; D’Anjou, Benjamin; Ghattamaneni, Nageswara; Harack, Benjamin; Hilke, Michael; Horth, Alexandre; Majlis, Norberto; Massicotte, Mathieu; Vandsburger, Leron; Whiteway, Eric; Yu, Victor (3 November 2011). "Experimental Review of Graphene" (PDF). ISRN Condensed Matter Physics. International Scholarly Research Network. 2012: 1-56.

[36] Charlier, J.-C.; Eklund, P.C.; Zhu, J.; Ferrari, A.C. (2008). Jorio, A.; Dresselhaus and, G.; Dresselhaus, M.S. (eds.). Electron and Phonon Properties of Graphene: Their Relationship with Carbon Nanotubes. Carbon Nanotubes: Advanced Topics in the Synthesis, Structure, Properties and Applications. Berlin/Heidelberg: Springer-Verlag.

Semenoff, G. W. (1984). "Condensed-Matter Simulation of a Three-Dimensional Anomaly". Physical Review Letters. 53 (26): 2449-2452.

[37] Wallace, P.R. (1947). "The Band Theory of Graphite". Physical Review. 71 (9): 622-634.

[38] Avouris, P.; Chen, Z.; Perebeinos, V. (2007). "Carbon-based electronics". Nature Nanotechnology. 2 (10): 605-15

[39] Morozov, S.V.; Novoselov, K.; Katsnelson, M.; Schedin, F.; Elias, D.; Jaszczak, J.; Geim, A. (2008). "Giant Intrinsic Carrier Mobilities in Graphene and Its Bilayer". Physical Review Letters. 100 (1): 016602.

[40] Berber, Savas; Kwon, Young-Kyun; Tománek, David (2000). "Unusually High Thermal Conductivity of Carbon Nanotubes". Phys. Rev. Lett. 84(20): 4613-6

[41] Balandin, A. A.; Ghosh, Suchismita; Bao, Wenzhong; Calizo, Irene; Teweldebrhan, Desalegne; Miao, Feng; Lau, Chun Ning (20 February 2008). "Superior Thermal Conductivity of Single-Layer Graphene". Nano Letters. 8 (3): 902-907.

[42] Cai, Weiwei; Moore, Arden L.; Zhu, Yanwu; Li, Xuesong; Chen, Shanshan; Shi, Li; Ruoff, Rodney S. (2010). "Thermal Transport in Suspended and Supported Monolayer Graphene Grown by Chemical Vapor Deposition". Nano Letters. 10 (5): 1645-1651.

[43] Zhu, Shou-En; Yuan, Shengjun; Janssen, G. C. A. M. (1 October 2014). "Optical transmittance of multilayer graphene". EPL. 108 (1): 17007. 
[44] Nair, R. R.; Blake, P.; Grigorenko, A. N.; Novoselov, K. S.; Booth, T. J.; Stauber, T.; Peres, N. M. R.; Geim, A. K. (6 June 2008). "Fine Structure Constant Defines Visual Transparency of Graphene". Science. 320 (5881):

[45] Kuzmenko, A. B.; Van Heumen, E.; Carbone, F.; Van Der Marel, D. (2008). "Universal infrared conductance of graphite". Physical Review Letters. 100 (11): 117401.

[46] K Yan; H Peng; Y Zhou; H Li; Z Liu (2011). "Formation of bilayer Bernal graphene: layerby-layer epitaxy via chemical vapor deposition". Nano Lett. 11 (3): 1106-10.

[47] Z Liu; K Suenaga PJF Harris; S lijima (2009). "Open and closed edges of graphene layers". Phys. Rev. Lett. 102 (1): 015501.

[48] Min, Lola; Hovden, Robert; Huang, Pinshane; Wojcik, Michal; Muller, David A.; Park, Jiwoong (2012). "Twinning and Twisting of Tri- and Bilayer Graphene". Nano Letters. 12 (3): $1609-1615$.

[49] E. Mostaani, N. D. Drummond and V. I. Fal'ko (2015). "Quantum Monte Carlo Calculation of the Binding Energy of Bilayer Graphene". Phys. Rev. Lett. 115 (11): 115501.

[50] Y Cao, V Fatemi, A Demir,, S Fang, SL Tomarken, JY Luo, J D Sanchez-Yamagishi, K Watanabe, T Taniguchi, E Kaxiras, R C Ashoori, P Jarillo-Herrero (2018). "Correlated insulator behaviour at half-filling in magic-angle graphene superlattices". Nature. 556 (7699): 80-84.

[51] Lu, Xiaobo; Stepanov, Petr; Yang, Wei; Xie, Ming; Aamir, Mohammed Ali; Das, Ipsita; Urgell, Carles; Watanabe, Kenji; Taniguchi, Takashi; Zhang, Guangyu; Bachtold, Adrian; MacDonald, Allan H.; Efetov, Dmitri K. (2019). "Superconductors, orbital magnets and correlated states in magic-angle bilayer graphene". Nature. 574 (7780): 653-657. [52] Watanabe, K., Taniguchi, T. \& Kanda, H. Direct-bandgap properties and evidence for ultraviolet lasing of hexagonal boron nitride single crystal. Nat. Mater. 3, 404-409 (2004). [53] Zhuang, H. L., Singh, A. K. \& Hennig, R. G. Computational discovery of single-layer III-V materials. Phys. Rev. B 87, 165415 (2013).

Singh, A. K., Zhuang, H. L. \& Hennig, R. G. Ab initio synthesis of single-layer III-V materials. Phys. Rev. B 89, 245431 (2014).

[54] Singh, A. K. \& Hennig, R. G. Computational synthesis of single-layer GaN on refractory materials. Appl. Phys. Lett. 105, 051604 (2014). 
[55] Di Carlo, A. (2001). "Tuning Optical Properties of GaN-Based Nanostructures by Charge Screening". Physica Status Solidi A. 183 (1): 81-85.

[56] Arakawa, Y. (2002). "Progress in GaN-based quantum dots for optoelectronics applications". IEEE Journal of Selected Topics in Quantum Electronics. 8 (4): 823-832. [57] Morkoç, H.; Strite, S.; Gao, G. B.; Lin, M. E.; Sverdlov, B.; Burns, M. (1994). "Largeband-gap SiC, III-V nitride, and II-VI ZnSe-based semiconductor device technologies". Journal of Applied Physics. 76 (3): 1363.

[58] Dora, Y.; Chakraborty, A.; McCarthy, L.; Keller, S.; Denbaars, S. P.; Mishra, U. K. (2006). "High Breakdown Voltage Achieved on AIGaN/GaN HEMTs with Integrated Slant Field Plates". IEEE Electron Device Letters. 27 (9): 713.

[59] Asif Khan, M.; Kuznia, J. N.; Bhattarai, A. R.; Olson, D. T. (1993). "Metal semiconductor field effect transistor based on single crystal GaN". Applied Physics Letters. 62 (15): 1786. [60] Davis, Sam (March 2010). "Enhancement Mode GaN MOSFET Delivers Impressive Performance". Power Electronic Technology. 36(3).

[61] "Gallium Nitride-Based Modules Set New 180-Day Standard For High Power Operation." Northrop Grumman, 13 April 2011 


\section{Chapter 2: Calculation of the transmission probability and tunneling current}

Since quantum theory was founded in 1920s, it has developed quite far away and led people to a new field that totally different from the classical ones. As the size of an object decrease to a certain level, the classical physical law would be invalid and quantum mechanic would become the ruler of object behaviors. The property under quantum mechanic achieves functions that never been seen before. So scientist would invent new materials and devices with a property that never found before. This is the reason that new materials and devices have been emerging due to vast developments in nanotechnologies. The aim of this project is to simulate the electron transport in quantum region based on the Schrodinger equation for random shaped energy barriers, which is very basic to quantum mechanics. The method of simulation is matlab coding. The simulation will help in understanding the concept of quantum field and applications in design and analysis of nanometer scale devices and systems. In this thesis, the section of introduce would give the background knowledge of this project, with the concept of quantum tunneling, wave function and Schrödinger equation involved. The section of methodology and discussion is the main part of the thesis. In these two section the method used in the project is listed and explained. The results from matlab coding would be compared and analyzed. The section of application would introduce two example that the tunneling current is involved and how others use the method in this project.

\subsection{Introduction}

In order to achieve the goal of this project, some concept must be introduced first. 


\subsubsection{Quantum tunneling}

Quantum tunneling describes one phenomenon of quantum mechanics. It was developed from the study of radioactivity [1]. It refers to the phenomenon that a particle could pass through a barrier that it classically could not. This is a phenomenon that could only happen under nano scale. Under nano scale, the behavior of a particle is quite different from that under classical scale. In classical mechanics, when a particle meets a barrier, if the particle attempts to pass the potential barriers, it must contain higher energy than the barrier so that it could climb and pass by, otherwise it just fail. It may be reflected by the barrier or even absorbed. However, in quantum mechanics, the particle could pass through the barrier and tunnel to the other side with a very small probability. This phenomenon that a particle tunnel through the barrier under quantum circumstance is called quantum tunneling. The mechanic of quantum tunneling could be explained by the energy-time uncertainty. Assuming that the original energy of the particle is $E$, the potential of the potential barrier is $V$, and $E<V$, the particle cannot pass the barrier classically. According to the energy-time uncertainty principle,

$$
\Delta E \Delta t \approx \hbar / 2(2.1)
$$

here $\Delta E$ is the uncertainty of energy and $\Delta t$ is time respectively, and $\hbar$ is the reduced Planck constant.

Although in classical mechanics, the total energy cannot be changed, otherwise, the law of conservation of energy will be violated. However, in quantum mechanics, if the uncertainty of time is $\Delta t$, the uncertainty of energy is $\Delta E$. Suppose that the particle temporarily borrows energy $\Delta E$ so that the total energy $\mathrm{E}+\Delta E>\mathrm{V}$, and then the particle can pass the barrier. However in order not to violate the energy-time uncertainty principle, the particle must return energy $\Delta E$ within time $\Delta t \approx \hbar /(2 \Delta E)$, and the particle must be pass the barrier in time, otherwise it still fail to pass.

\subsubsection{Wave function}


The quantum tunneling can be described by a wave function. A wave function is a function that describes the quantum state of an isolated system with one or more particles in quantum mechanics. For one certain system, there is one wave function that contains all the information about it even if the system has more than one particle. This is due to the probability amplitude of the system. Probability amplitude provides a relationship between the wave function of a system and the results of observations. So any quantities that are associated with measurements, such as the average speed or position of a particle, can be got from the wave function. This determines the core role in the quantum mechanics and the reason why it important in all modern theories.

For a given system, the wave function is set by deciding a representation related to a maximal set of commuting observables and a suitable coordinate system. Once the wave function is set, it is a complex-valued function of the system's degrees of freedom corresponding to the chosen representation and coordinate system.

The Schrödinger equation is the most common wave function used today and it is the basic equation of this project.

\subsubsection{Schrödinger equation}

Schrödinger equation is a partial differential equation that describes how the quantum state of a physical system changes with time in quantum mechanics. It was named by the famous Austrian physicist Erwin Schrödinger. The equation was formulated in late 1925 and published in 1926[2]. Mainly there are two types of the equation: time-dependent and time-independent. This project is based on the solution of time-independent Schrödinger equation.

The time-dependent Schrödinger equation

$$
i \hbar \frac{\partial}{\partial x} \Psi(r, t)=\left[\frac{-\hbar^{2}}{2 \mu} \nabla^{2}+V(r, t)\right] \Psi(r, t)(2.2)
$$

This is the non-relativistic Schrödinger equation for a single particle. In this equation $\mathrm{i}$ is the imaginary unit, $\hbar$ is the reduced Planck constant which equals 
plank constant divided by $2 \pi$, $\partial / \partial$ t stands for a partial derivative, $\Psi$ is the wave function of the quantum system. $\mu$ is the particle's reduced mass, $V$ is the potential energy, $\nabla^{2}$ is the Laplacian (a differential operator). The timedependent Schrödinger equation is used to describe a system that changes with time.

The time-independent Schrödinger equation

$$
E \Psi(r)=\left[\frac{-\hbar^{2}}{2 \mu} \nabla^{2}+V(r)\right] \Psi(r)(2.3)
$$

This is the time-independent Schrödinger equation for a single non-relativistic particle. $\mathrm{E}$ here stands for energy eigenvalue, so the time independent Schrödinger equation is an eigenvalue equation. The wave function involved in time-independent Schrödinger equation describing waves with definite energy compared to those of a probability distribution of different energies. In physics, these standing waves are called "stationary states" or "energy eigenstates"; in chemistry they are called "atomic orbitals" or "molecular orbitals".

\subsection{Methodology}

The aim of this project is to calculate the transmission current under certain circumstance. In non-relativistic quantum mechanics, the transmission coefficient and related reflection coefficient are used to describe the behavior of waves incident on a barrier [3]. The transmission coefficient presents the probability of a particle tunneling through a barrier. Only when particles with charge tunnel trough a barrier here forms the tunneling current. So in order to get the transmission current, the transmission coefficient must be calculated first. This is the reason why the core of the project is to calculate the transmission coefficient.

In this project, the main method is to solve the time-independent Schrödinger with the help of scattering matrix and a concept of multistep. Compared to the 
conventional way of approximation or dealing with continuous variations of potential energy, the method here is to split the potential barrier up into $\mathrm{N}$ segments and in which the potential energy can be regarded as a constant. So as $\mathrm{N}$ increased, the segments become finer and finer, finally a continuous variation will be restored. The transmission coefficient is calculated by a method to connect momentum eigenfunctions[4].

\subsubsection{WBK Method}

Firstly the WBK approximation would be introduced. WBK here stands for Wentzel, Kramers, and Brillouin, which are the names of three physicists who developed this method. The WKB approximation is the conventional method to calculate the transmission coefficient across potential barriers. If the potential is a constant or changing very slowly, the wavefunction solutions of the 1-D

Schrodinger equation $E \Psi(r)=\left[\frac{-\hbar^{2}}{2 u} \nabla^{2}+V(r)\right] \Psi(r)$ are of the form of a simple plane wave $\psi(x)=A \exp ( \pm i k x)$ and $k=\sqrt{2 m^{*}(E-U)} / \hbar$. The transmission coefficient is express as:

$$
T=\frac{\exp \left(-2 \int_{x_{1}}^{x_{2}} d x \sqrt{\frac{2 m}{\hbar^{2}}(V(x)-E)}\right.}{\left(1+\frac{1}{4} \exp \left(-2 \int_{x_{1}}^{x_{2}} d x \sqrt{\frac{2 m}{\hbar^{2}}(V(x)-E)}\right)^{2}\right.}(2.4)
$$

where $m$ is effective mass and $V(x)$ is potential and $E$ is energy of electron. However, wbk is typically used for a semi classical calculation in quantum mechanics and has some limitation in application.

\subsubsection{Multistep}




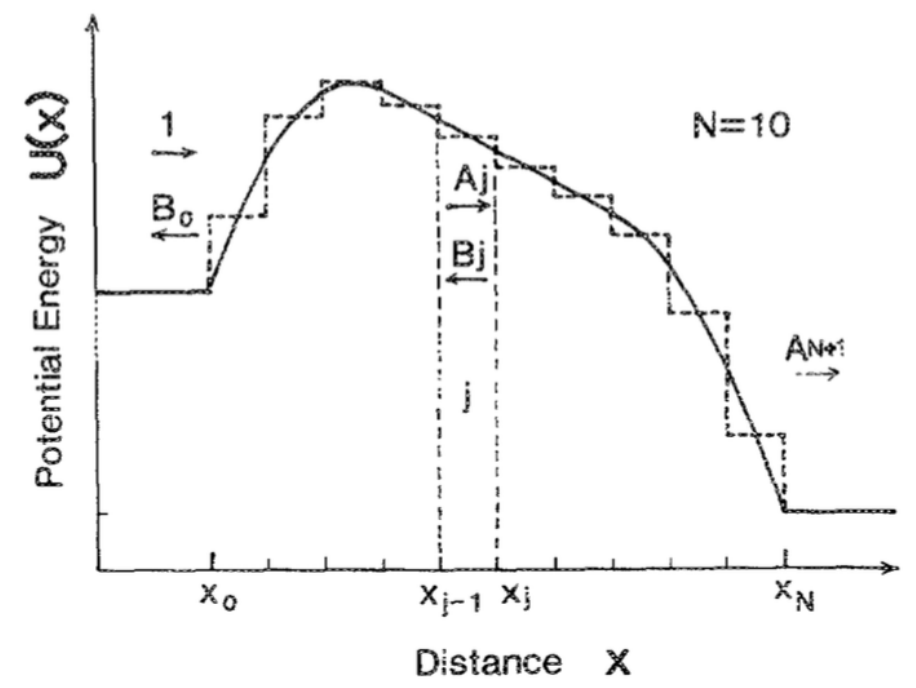

Figure 2.1 One example of potential energy $U(x)$ vs distance $X[5]$

This is the main method involved in this project. An simple instance is taken to explain the method. Figure 1 shows potential Energy $U(x)$ versus distance $x$. The first step is to divide the barrier part into $\mathrm{N}$ segments(Here in the example $\mathrm{N}=10$ ), then it can be drawn from the figure that the potential barrier is:

$$
U(x)=U\left[\left(x_{j-1}+x_{j}\right) / 2\right](2.5)
$$

And the effective mass is expressed as:

$$
m^{*}(x)=m_{j}=m^{*}\left[\left(x_{j-1}+x_{j}\right) / 2\right](2.6)
$$

for $x_{j-1}<x<x_{j}$ and $\mathrm{j}=0,1,2, \ldots \mathrm{N}, \mathrm{N}+1$

Because the potential is treated as constant in each segment, the solution to the one-dimensional Schrodinger equation is given in the jth step as a superposition of plane waves:

For the wave function $\Psi$ in the $J_{t h}$ step:

$$
\psi_{j}(x)=A_{j} \exp \left(i k_{j} x\right)+B_{j} \exp \left(-i k_{j} x\right)(2.7)
$$




$$
k_{j}=\sqrt{2 m_{j}^{*}\left(E-U_{j}\right)} / \hbar(2.8)
$$

Because of the continuity of $\psi_{\mathrm{j}}(\mathrm{x})$ and $\left(1 / \mathrm{m}_{\mathrm{j}}^{*}\right)^{\star}\left(\mathrm{d} \psi_{\mathrm{j}} / \mathrm{dx}\right)$ at the boundary of step $\mathrm{j}$ and $\mathrm{J}+1$, the process to determine the factor $A_{j}$ and $B_{j}$ in Eqs. (2.7) can just be simplified to the multiplication of the following $N+1(2 \times 2)$ matrices:

$$
\left(\begin{array}{l}
A_{j} \\
B_{j}
\end{array}\right)=\prod_{l=0}^{j=l} M_{l}\left(\begin{array}{l}
A_{0} \\
B_{0}
\end{array}\right)(2.9)
$$

and

$$
M_{l}=\frac{1}{2}\left[\begin{array}{cc}
\left(1+S_{l}\right) \exp \left[-i\left(k_{l+1}-k_{l}\right) x_{l}\right] & \left(1-S_{l}\right) \exp \left[-i\left(k_{l+1}+k_{l}\right) x_{l}\right] \\
\left(1-S_{l}\right) \exp \left[i\left(k_{l+1}+k_{l}\right) x_{l}\right] & \left(1+S_{l}\right) \exp \left[i\left(k_{l+1}-k_{l}\right) x_{l}\right]
\end{array}\right]
$$

and

$$
S_{l}=\frac{m_{l+1}^{*}}{m_{l}^{*}} \frac{k_{l}}{k_{l+1}}(2.11)
$$

However in common situation the effective mass would not change with distance $x$ (for example in MOSFET made of silicon), so that (2.11) could be simplified as

$$
S_{l}=\frac{k_{l}}{k_{l+1}}\left(2.11^{*}\right)
$$

When $\mathrm{j}=\mathrm{N}+1$, Eq. (2.9) becomes

$$
\left(\begin{array}{l}
A_{N+1} \\
B_{N+1}
\end{array}\right)=\prod_{l=0}^{l=N+1} M_{l}\left(\begin{array}{l}
A_{0} \\
B_{0}
\end{array}\right)(2
$$

Set $A_{0}=1$ and $B_{N+1}=0$, which means an electron incident from the left hand side of Fig 1 and generates left-justified states [2.10] in physic, the transmission amplitude $A_{N+1}$ can be calculated by the equation:

$$
\begin{gathered}
A_{N+1}=\frac{m_{N+1}^{*}}{m_{0}^{*}} \frac{k_{0}}{k_{N+1}} \frac{1}{M_{22}}(2.13) \\
M=\left[\begin{array}{ll}
M_{11} & M_{12} \\
M_{21} & M_{22}
\end{array}\right]=\prod_{l=0}^{N} M_{l}(2.14)
\end{gathered}
$$

Finally the transmission probability $D(E, V)$ can be calculated by following:

$$
D\left(E_{x}, V_{b}\right)=\frac{m_{0}^{*}}{m_{N+1}^{*}} \frac{k_{N+1}}{k_{0}}\left|A_{N+1}\right|^{2}(2
$$


Applying the formula of (2.11*) (2.13)and(2.14), (2.15) could be rewritten as:

$$
D\left(E_{x}, V_{b}\right)=\frac{k_{0}}{k_{N+1}}\left|\frac{1}{M_{22}}\right|^{2}(2
$$

$E_{x}$ here stand for the incident energy of electron and $V_{b}$ is the voltage applied.

Once the transmission probability is got, the transmission current density is given by the Tsu-Esaki current formula:

$$
J\left(E_{x}\right)=\frac{q m_{0}^{*} k T}{2 \pi^{2} \hbar^{3}} \times \int_{0}^{\infty} D\left(E_{x}, V_{b}\right) \times \ln \left(\frac{1+\exp \left(E_{f}-E_{x}\right) / k T}{1+\exp \left(E_{f}-q V_{b}-E_{x}\right) / k T}\right) d\left(E_{x}\right)(2.17)
$$

where $\mathrm{q}$ is the electron charge, $m_{0}^{*}$ is the electron effective mass. $\mathrm{k}$ is the Boltzmann constant, $T$ is the temperature, and $E_{f}$ is the Fermi energy. So, $J$ is a function of transmission probability, then become the function of $E_{x}$ and $V_{b}$.

Besides the method introduced above, there are other ways to get the transmission coefficient.

\subsubsection{Propagation Matrix}

This method is quite similar to the multistep method explained above, however it focuses on the relation of wavefunction that present the particles pass into and out of a barrier and travel inside the barrier. Here using an example to explain the method: 


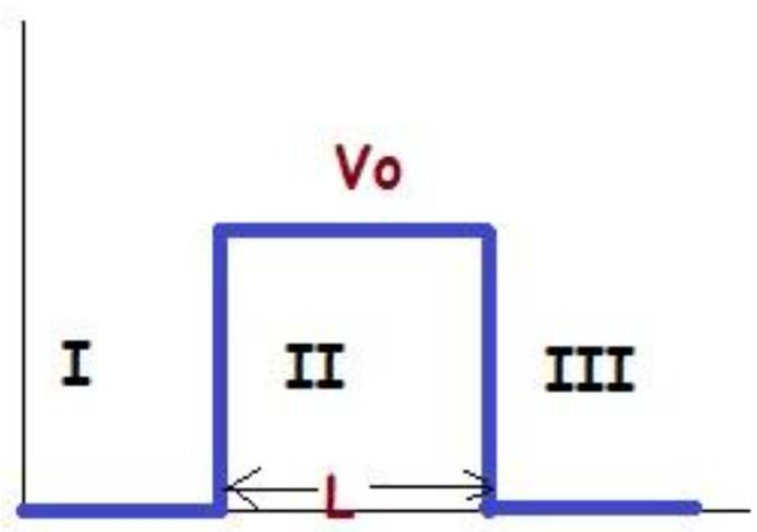

Figure 2.2 A potential barrier with height of $V_{0}$ and width of $L$

In figure 2 there is a potential barrier with the height of $V_{0}$ and width of L. I, II, and III here indicate three regions that a particle would pass into, travel through and pass out of the barrier. Since the barrier is a rectangular one, so the Schrödinger equation can be simplified. The wavefunction of the three regions is:

$$
\begin{aligned}
\Psi_{I}(x) & =A \exp \left(i k_{1} x\right)+B \exp \left(-i k_{1} x\right)(2.18) \\
\Psi_{I I}(x) & =C \exp \left(i k_{2} x\right)+D \exp \left(-i k_{2} x\right)(2.19) \\
\Psi_{I I I}(x) & =F \exp \left(i k_{3} x\right)+G \exp \left(-i k_{3} x\right)(2.20)
\end{aligned}
$$

where $k_{1}=k_{3}=\frac{\sqrt{2 m E}}{\hbar}, k_{2}=\frac{\sqrt{2 m\left(E-V_{0}\right)}}{\hbar}, \mathrm{m}$ is mass of electron and $\mathrm{E}$ is energy of electron.

The propagation matrix is one matrix that relates the waves on the right hand side to those on the left of the barrier. Here, the matrix for left $(P I)$, in barrier $(\mathrm{Pb})$ and right $(\mathrm{Pr})$ is expressed as below

$$
\begin{gathered}
P l=\frac{1}{2 \sqrt{k_{1} k_{2}}}\left(\begin{array}{cc}
k_{1}+k_{2} & k_{1}-k_{2} \\
k_{1}-k_{2} & k_{1}+k_{2}
\end{array}\right)(2.21) \\
P b=\left(\begin{array}{cc}
e^{-i k_{2} L} & 0 \\
0 & e^{i k_{2} L}
\end{array}\right)(2.22) \\
P r=\frac{1}{2 \sqrt{k_{1} k_{2}}}\left(\begin{array}{ll}
k_{1}+k_{2} & k_{1}-k_{2} \\
k_{1}-k_{2} & k_{1}+k_{2}
\end{array}\right)(2.23)
\end{gathered}
$$

The propagation matrix is calculated by multiplying these three matrices together: $\mathrm{P}=\mathrm{PI} \times \mathrm{Pb} \times \mathrm{Pr}$. Since $\mathrm{PI}, \mathrm{Pb}$ and $\mathrm{Pr}$ are all $(2 \times 2)$ matrices, $\mathrm{P}$ is still a $(2 \times 2)$ matrix.

The transmission coefficient is defined as 


$$
T=\frac{1}{\left|P_{11}\right|^{2}}(2.24)
$$

Although the example above only use one barrier, if combining a series of barriers together, a real potential barrier could be achieved. However, the calculation and complexity would increase rapidly.

\subsubsection{Theoretical calculation}

This method is to calculation the transmission coefficient directly by solving 1D Schrödinger equation and make approximation. The transmission coefficient is defined as:

$$
T=\left[1+\frac{E_{p}^{2} \sinh ^{2}(k l)}{4 E\left(E-E_{p}\right)}\right]^{-1} \text { and } k=\frac{\sqrt{2 m\left|E_{p}-E\right|}}{\hbar}(2.25)
$$

$\mathrm{E}$ is particle energy, $E_{p}$ is height of potential barrier, I is width of barrier and $\mathrm{m}$ is the mass.

\subsection{Result analysis}

In this section the image generated by matlab would be listed and compared. Rectangular Barrier:

The barrier shape is: 


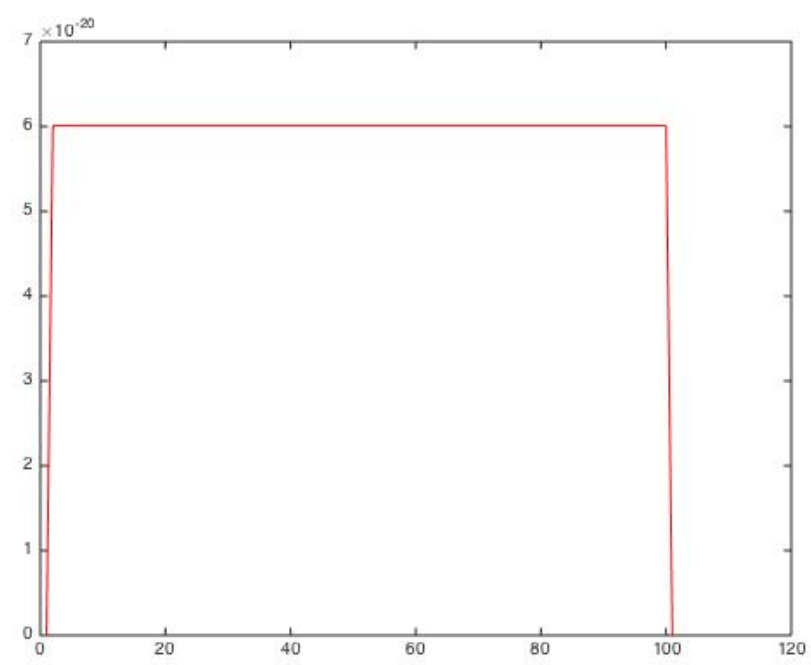

Following are the result of rectangular barrier with different step number $\mathrm{N}$, barrier height $\mathrm{U}(\mathrm{eV})$ and barrier width $\mathrm{L}(\mathrm{Ai})$

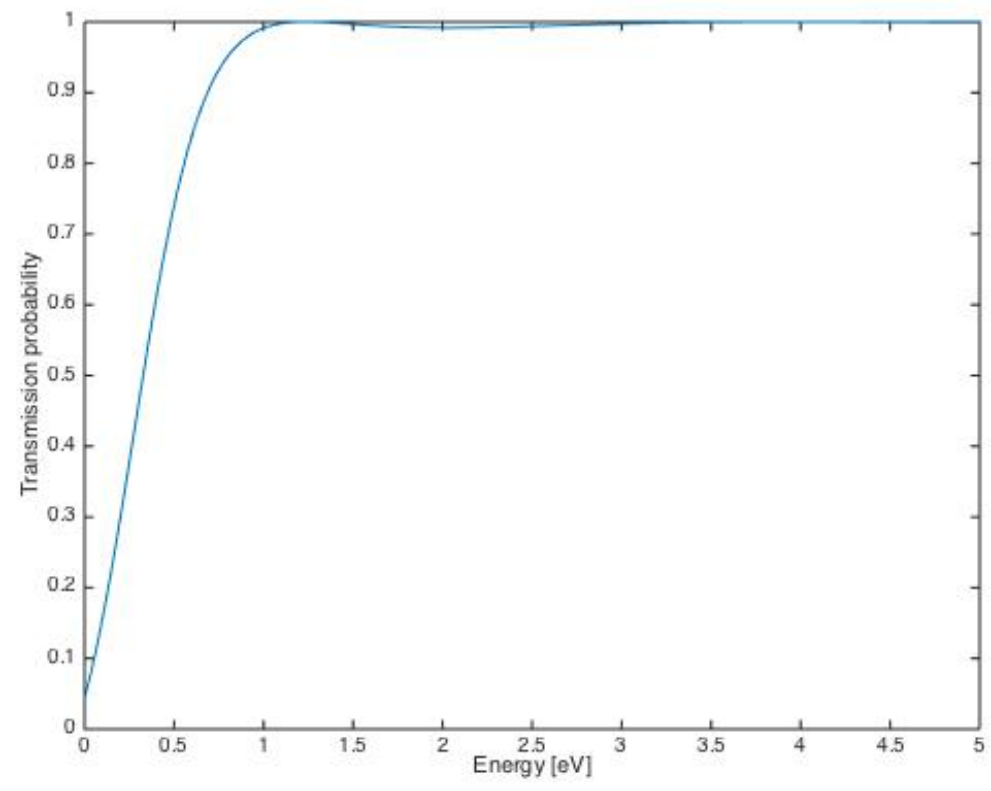

Figure $2.3 L=20 \mathrm{Ai}$ 


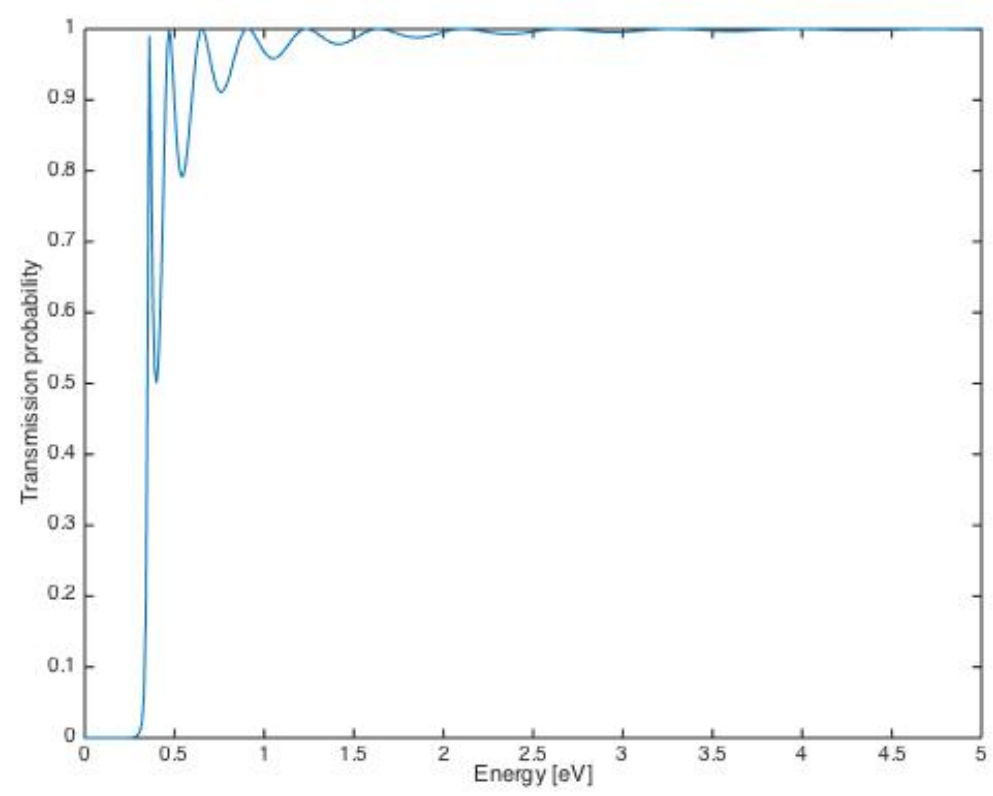

Figure $2.4 L=100 \mathrm{Ai}$

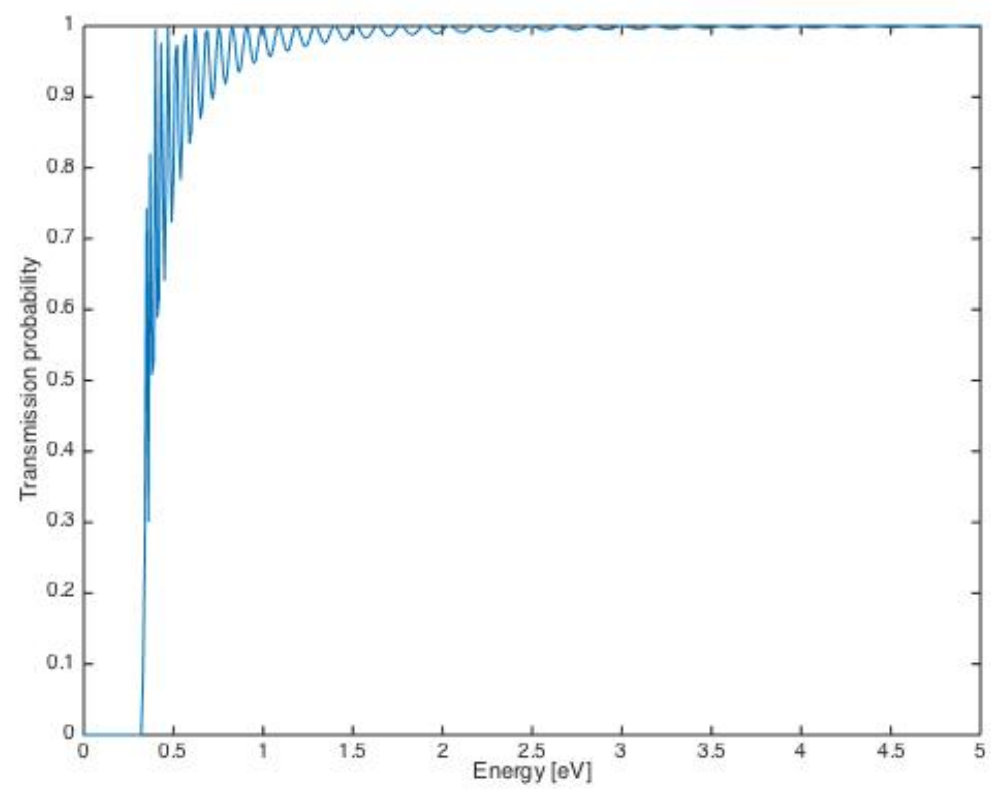

Figure $2.5 L=350 A i$

For figure 2.3,2.4 and 2.5, the barrier height and the step number are constant. The height is $0.375 \mathrm{ev}$ and step number is 100 . It is clear that the wider the barrier is, the wavier behavior in low energy area, which means harder to tunnel through. 


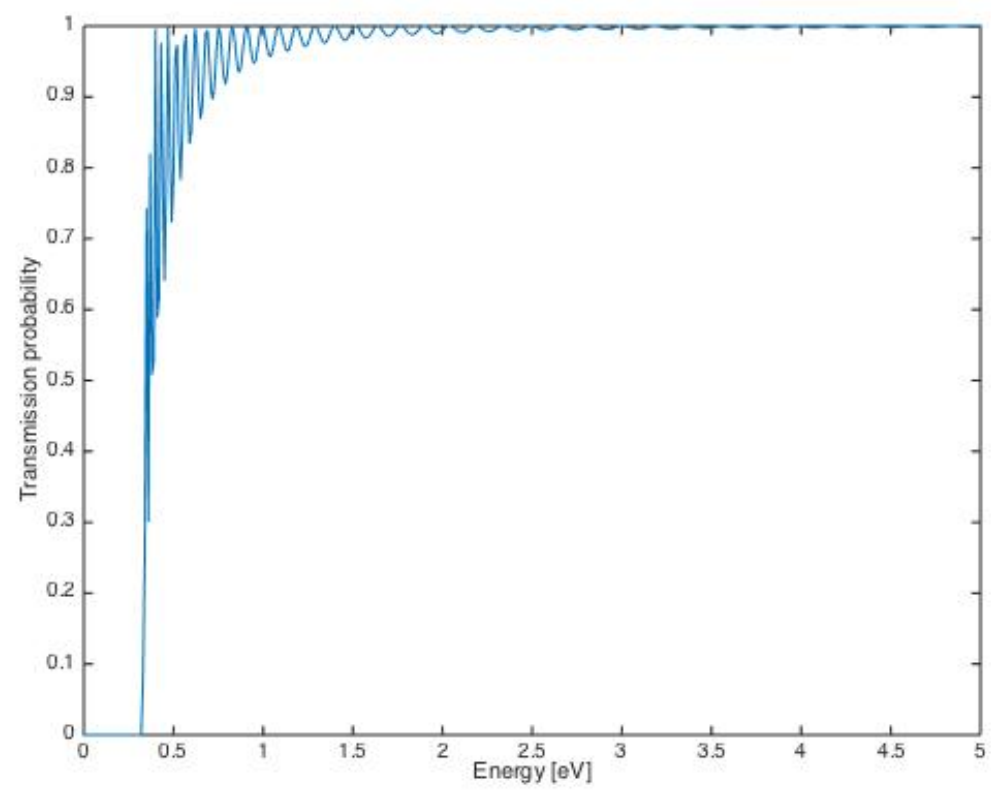

Figure $2.6 U=0.375 \mathrm{ev}$

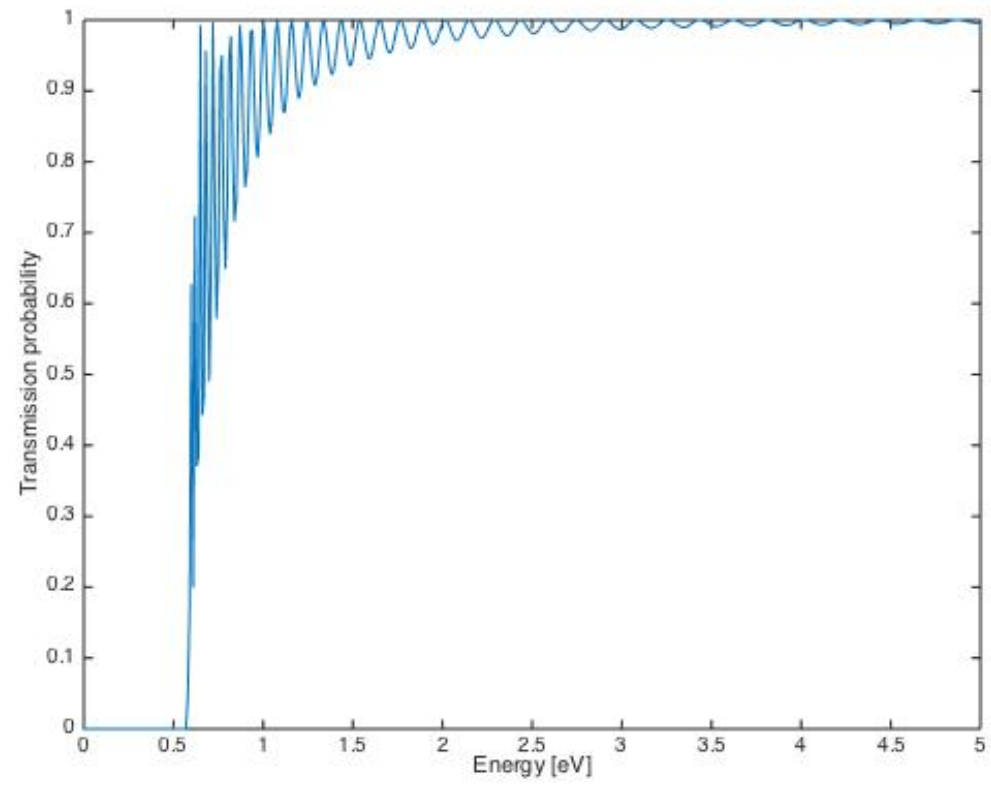

Figure $2.7 U=0.625 \mathrm{eV}$ 


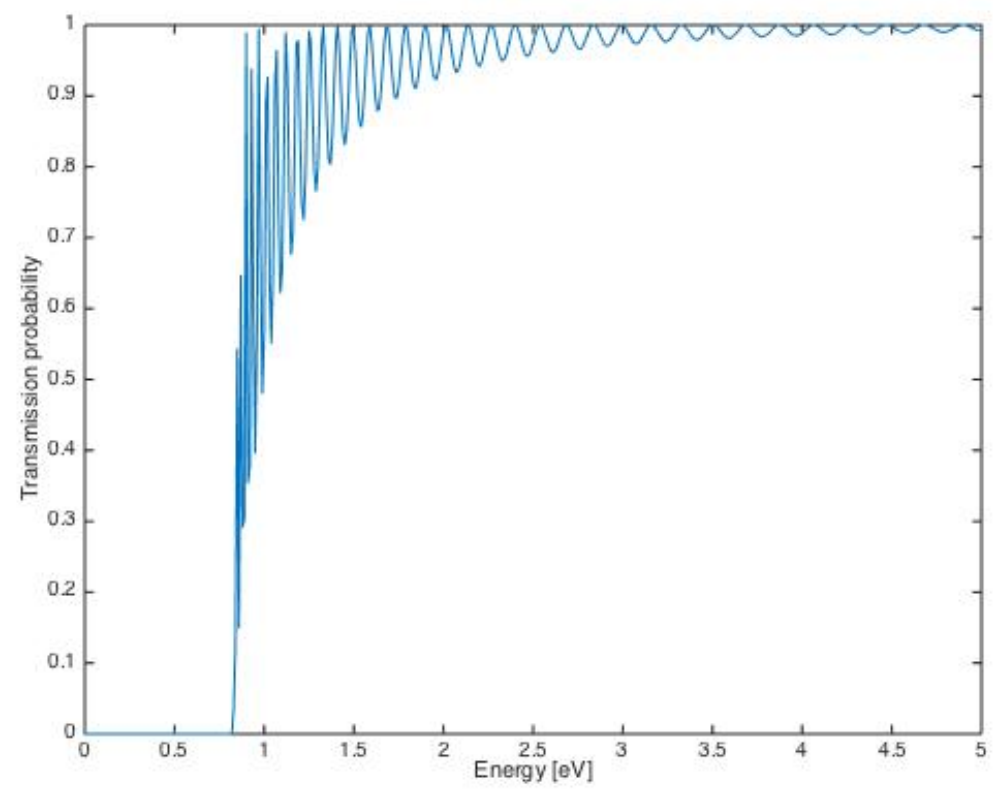

Figure $2.8 U=0.875 \mathrm{eV}$

For figure 2.6,2.7 and 2.8 barrier width and step number is constant. The width is 350Ai and step number is 100. Passing limit shifts towards higher energies on increasing barrier height. Wavy behavior is more prominently at higher barrier height and higher energies

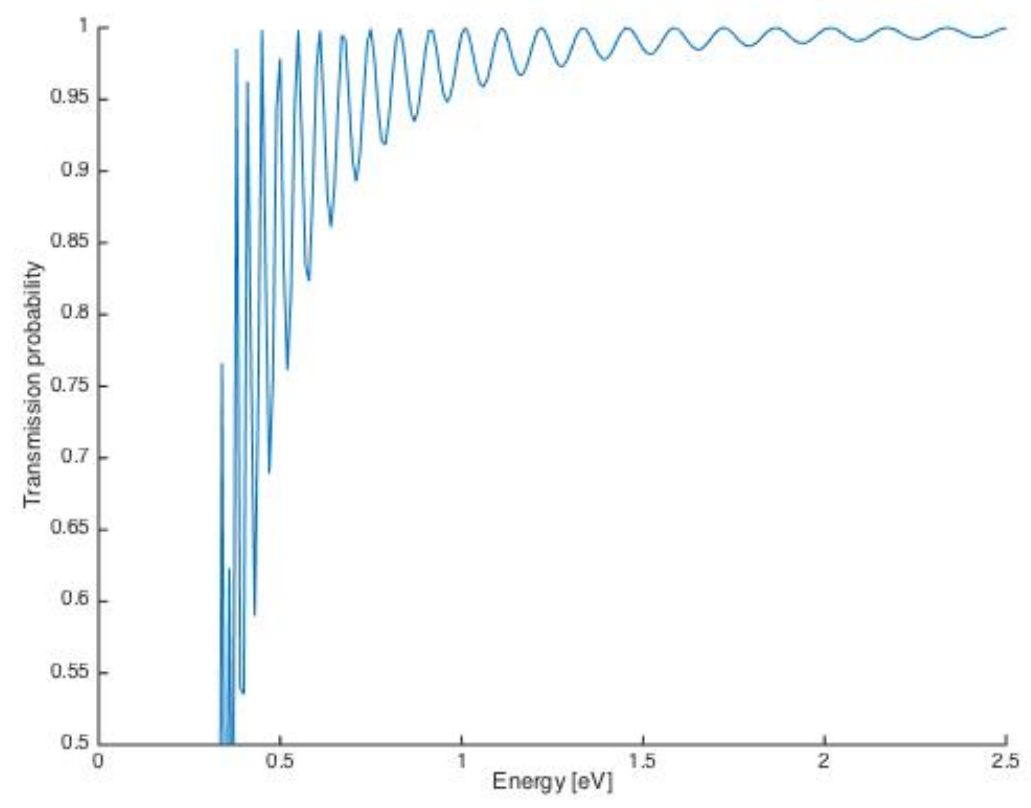

Figure $2.9 \mathrm{~N}=10$ 


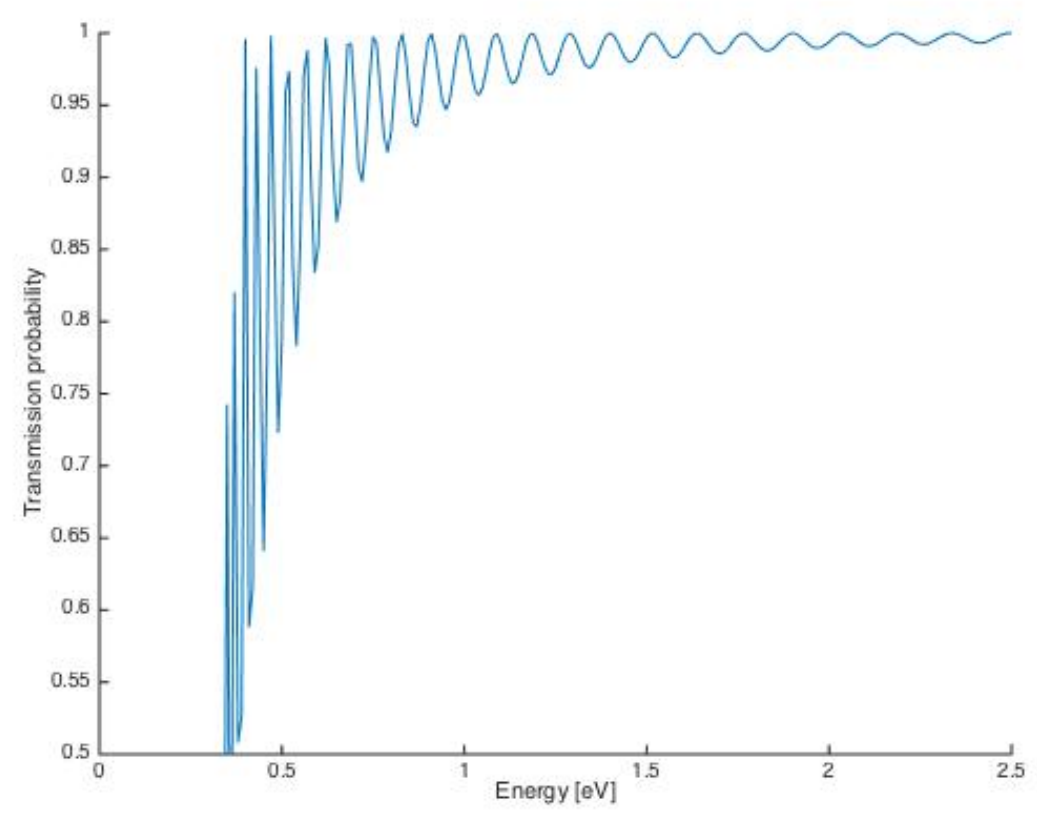

Figure $2.10 \mathrm{~N}=40$

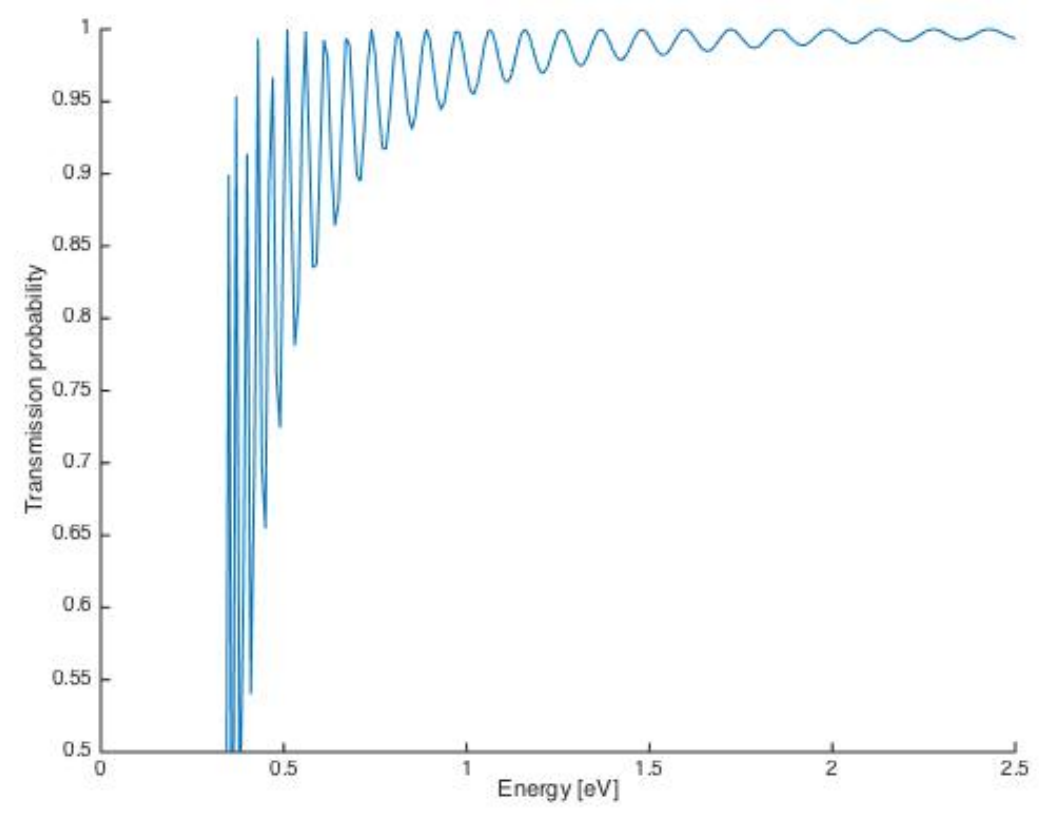

Figure $2.11 \mathrm{~N}=100$

For figure 2.9,2.10 and 2.11, barrier width(350Ai) and height(0.375ev) are constant. The only variable here is step number N. However, these three figure have slight difference from each other. It means that step number has little influence on rectangular barrier. This is because the potential for a rectangular barrier is constant whether the multistep method is applied or not. 


\section{Parabolic Barrier:}

The barrier shape is:

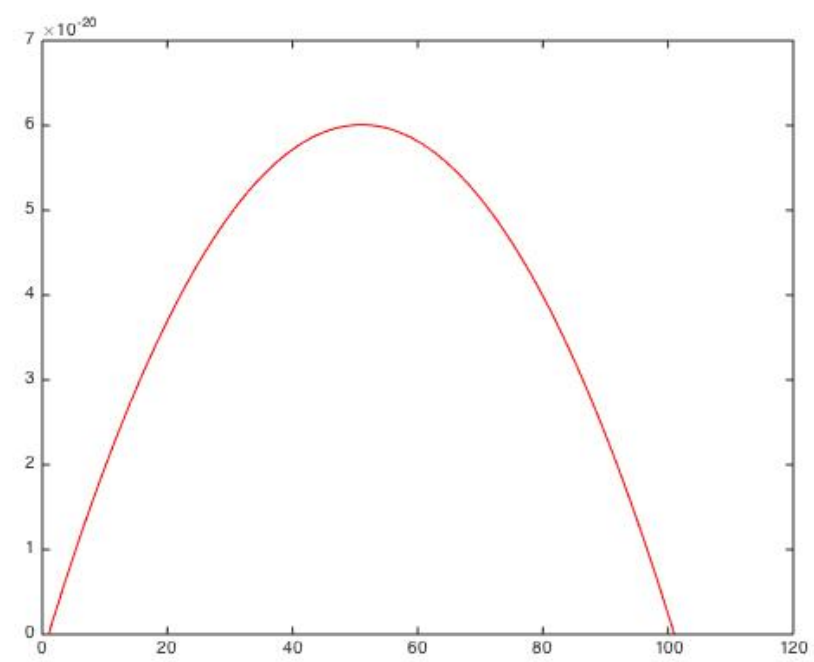

Now the result of parabolic barrier would be listed

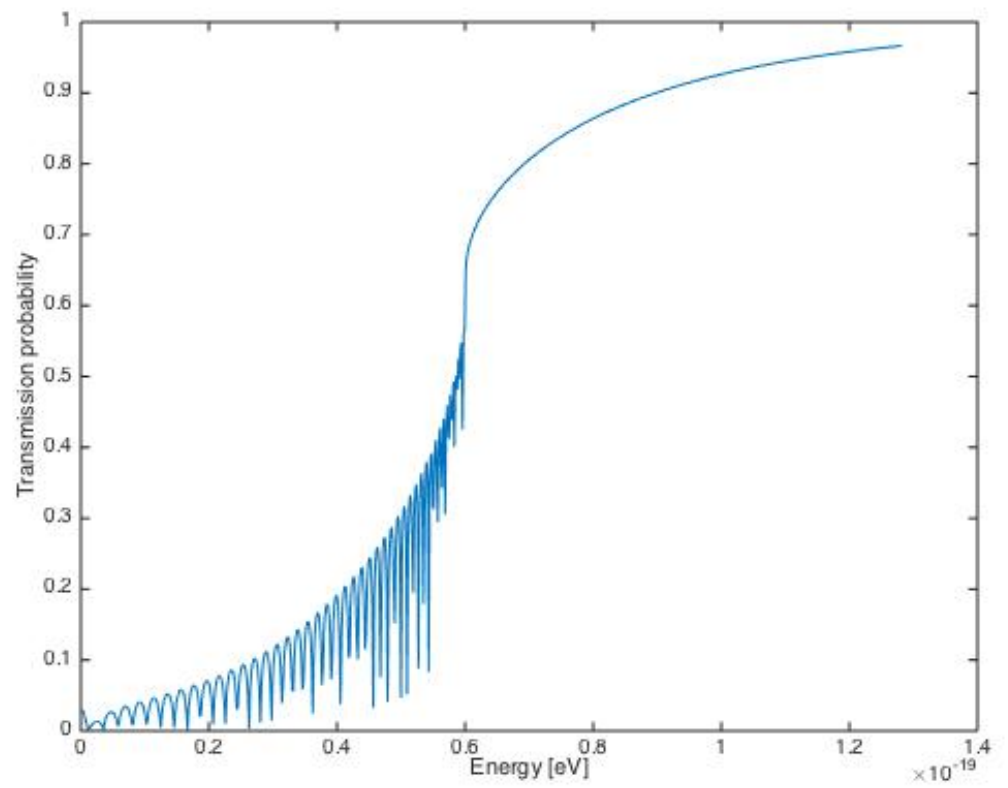

Figure $2.12 L=20 A i$ 


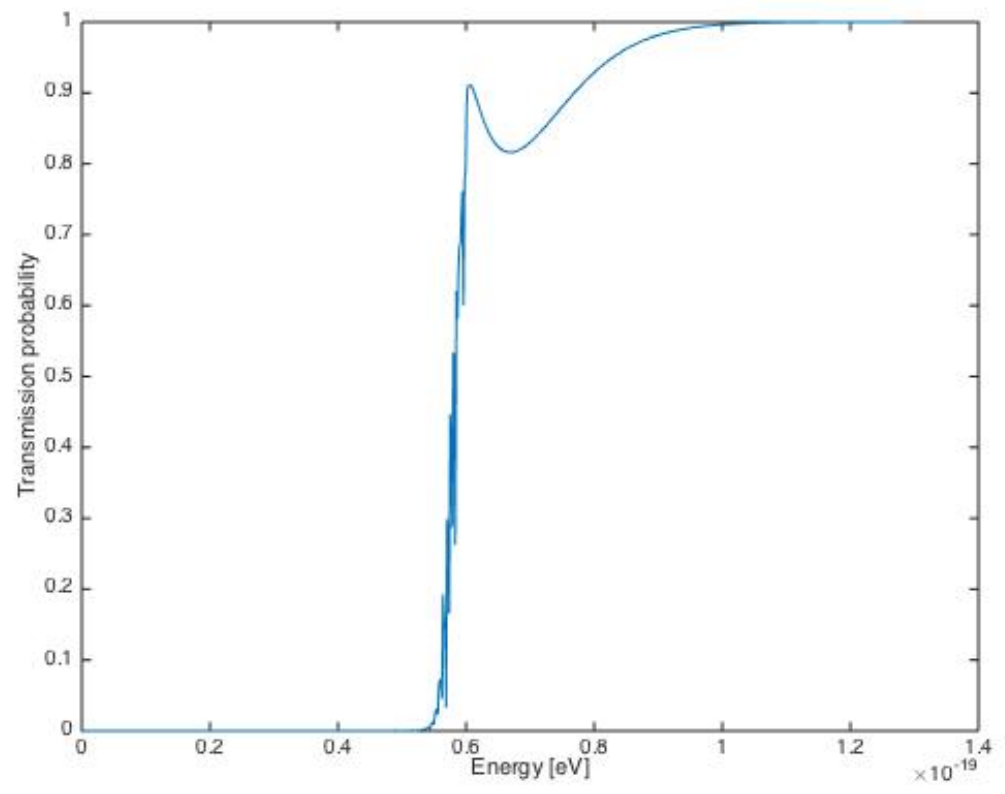

Figure $2.13 L=100 A i$

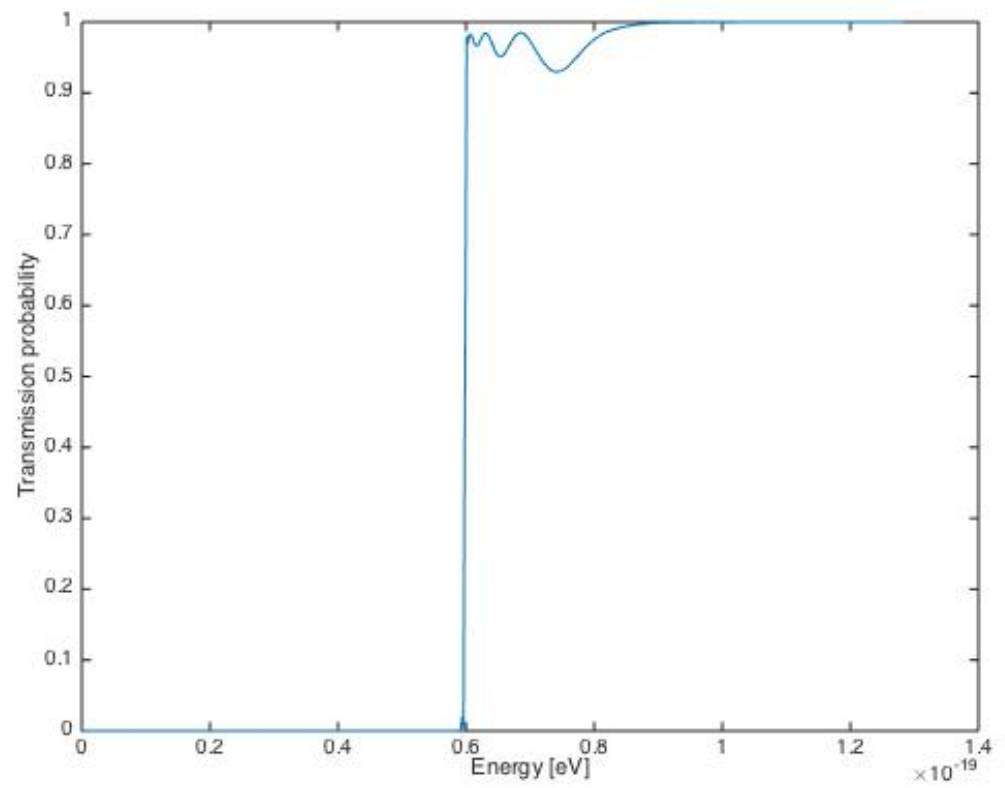

Figure $2.14 L=350 A i$

For figure 2.12,2.13 and 2.14 the variable is barrier width. The height is $0.375 \mathrm{ev}$ and step number is 100 . From the figure it is clear that the parabolic barrier is more sensitive to the barrier width. 


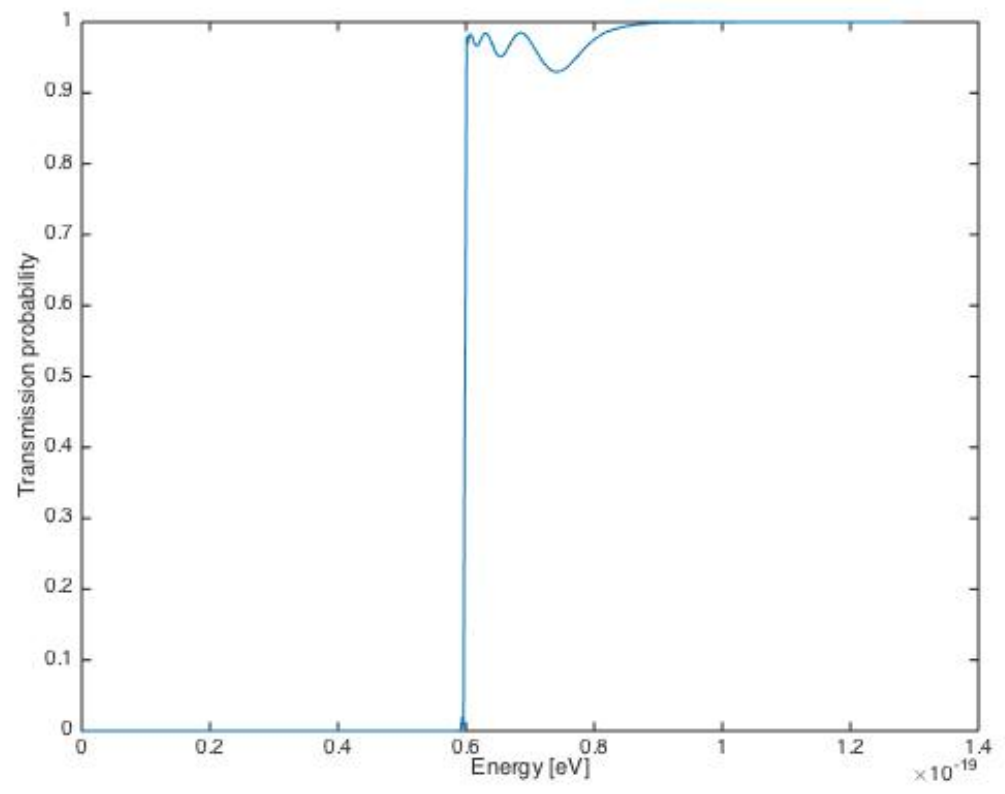

Figure $2.15 \mathrm{U}=0.375 \mathrm{ev}$

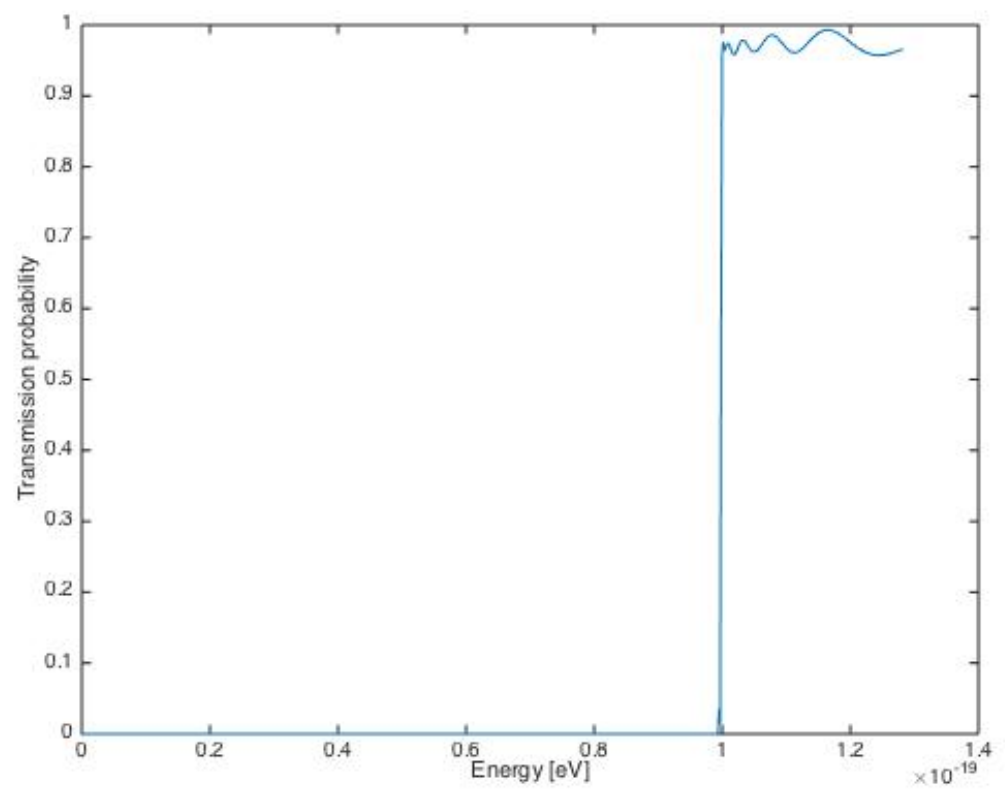

Figure $2.16 U=0.625 \mathrm{ev}$ 


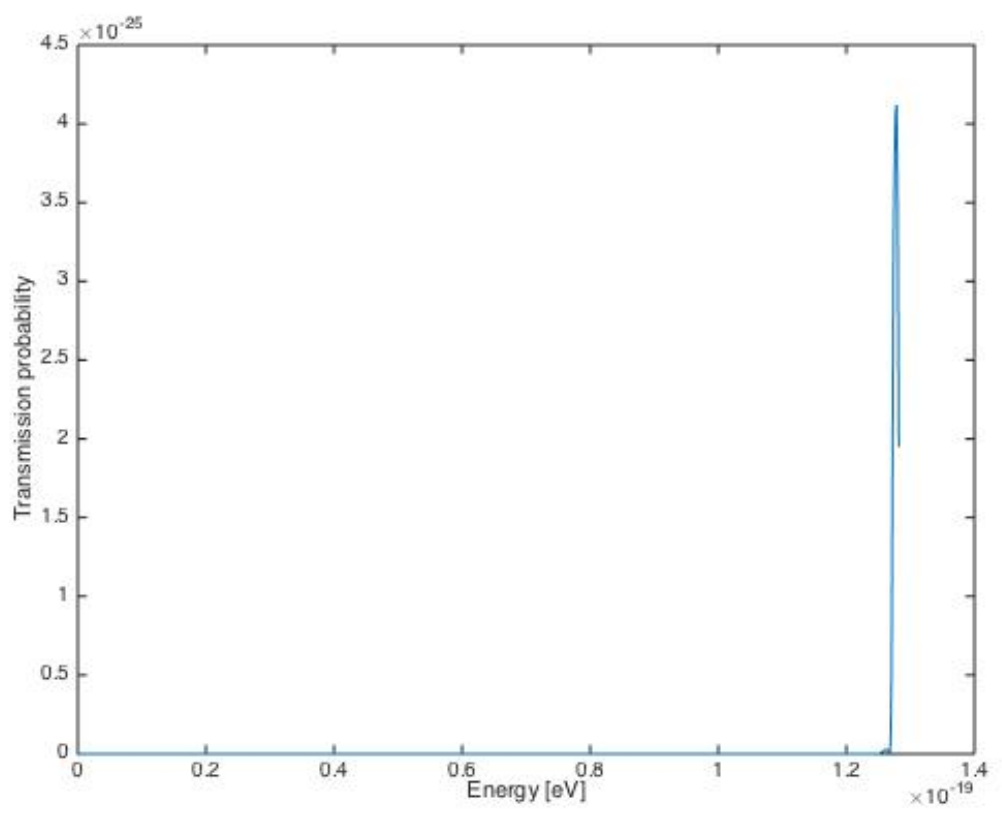

Figure $2.17 \mathrm{U}=0.875 \mathrm{eV}$

For figure 2.15,2.16 and 2.17 step number is 100 and barrier width is 350Ai. The barrier height has series influence on the probability. The higher the barrier is, the harder for the elctron to tunnel through.

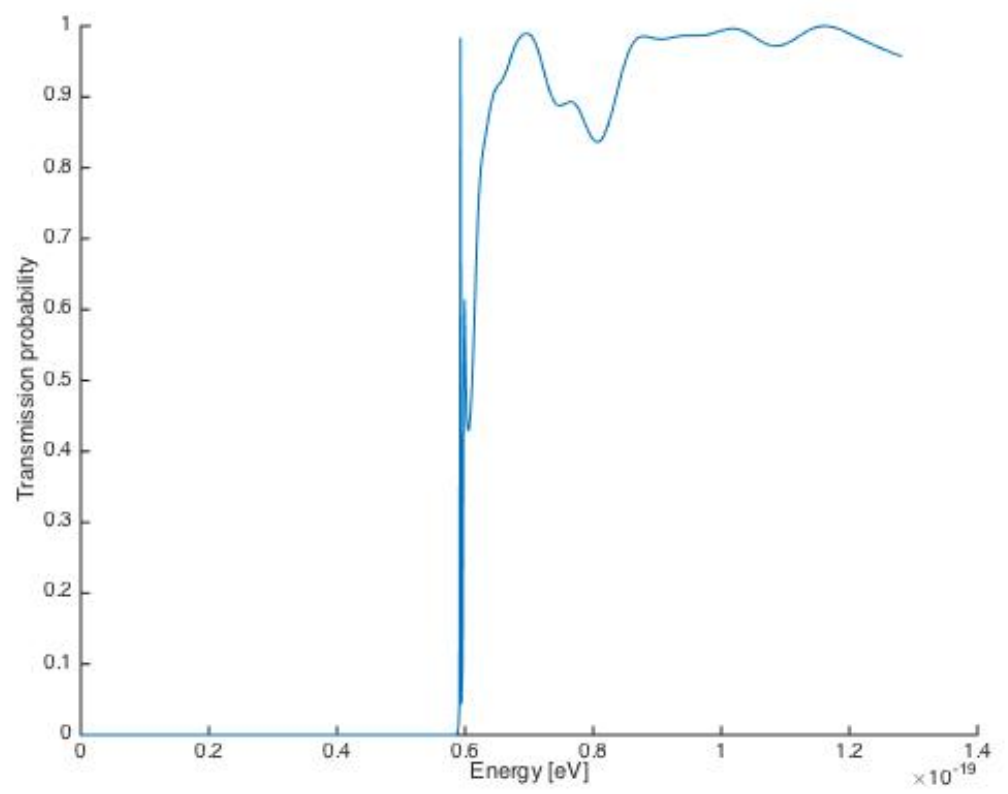

Figure $2.18 \mathrm{~N}=10$ 


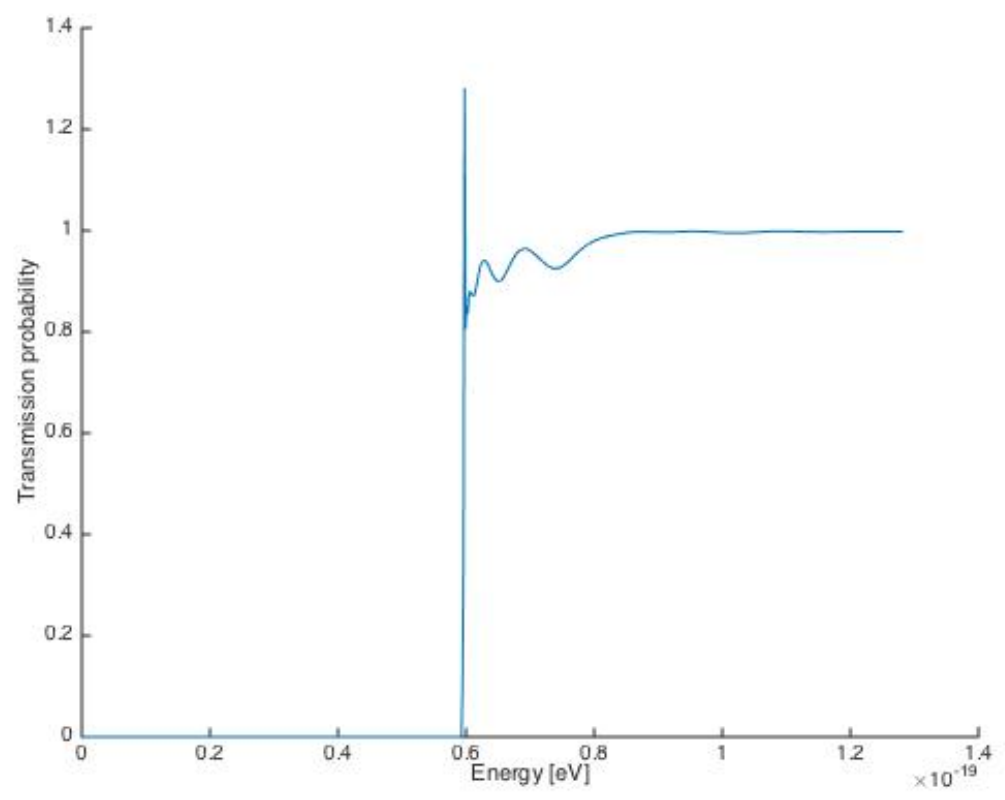

Figure $2.19 \mathrm{~N}=40$

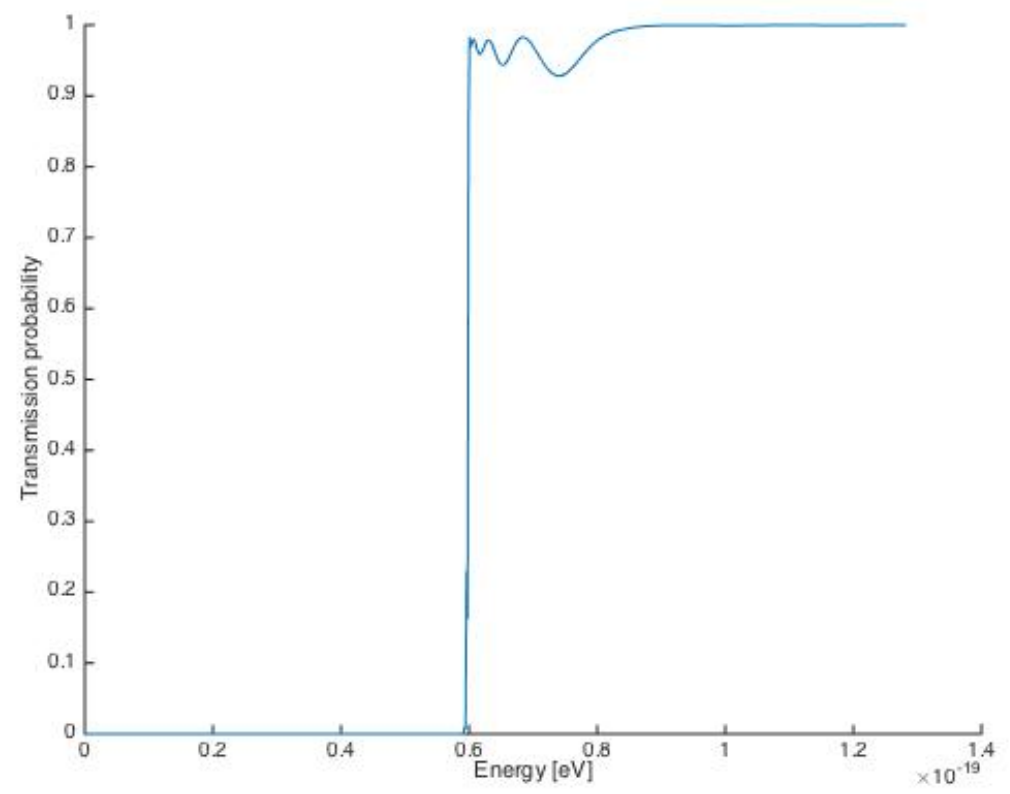

Figure $2.20 \mathrm{~N}=70$ 


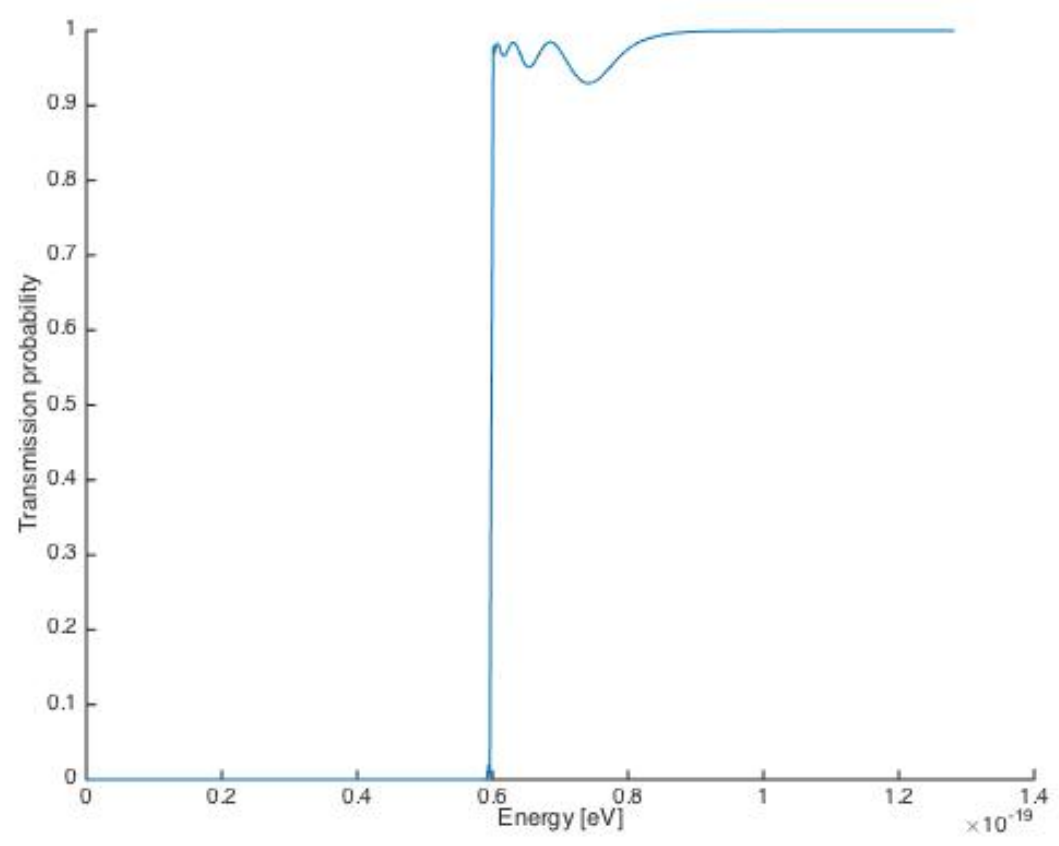

Figure $2.21 N=100$

For figure $2.18,2.19,2.20$ and 2.21 the barrier height is $0.375 \mathrm{ev}$ and barrier width is 350Ai. The step number is from 10 to 100 . There are four image because when $\mathrm{N}=40$, the maximum value of the probability is even bigger than 1! Any value around 40 would lead to the same result. Meanwhile the result works normally indicates that the program is well functioned. So it may be related to the method itself. From the four images, it tells that the step number has great influence on the result. However the result of 70 and 100 is almost the same. This indicates that to make sure the result is accurate, a minimum step value exist. This is because if the $\mathrm{N}$ is to small, the steps that combine together is still far from the real potential, making the result unacceptable.

\section{Rectangular well:}

The rectangular well here is made of two material: GaAs and AlAs, so the effective mass changes with distance $\mathrm{x}$, just as the figure 2.3 shows: 


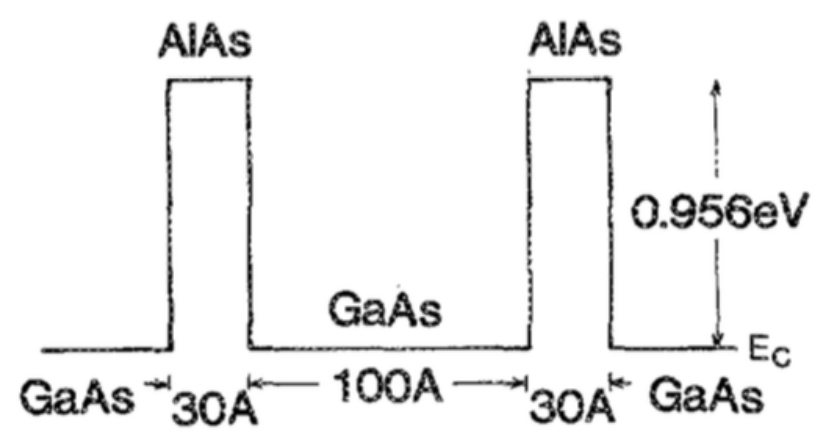

Figure 2.22 Rectangular well

The potential shape is as

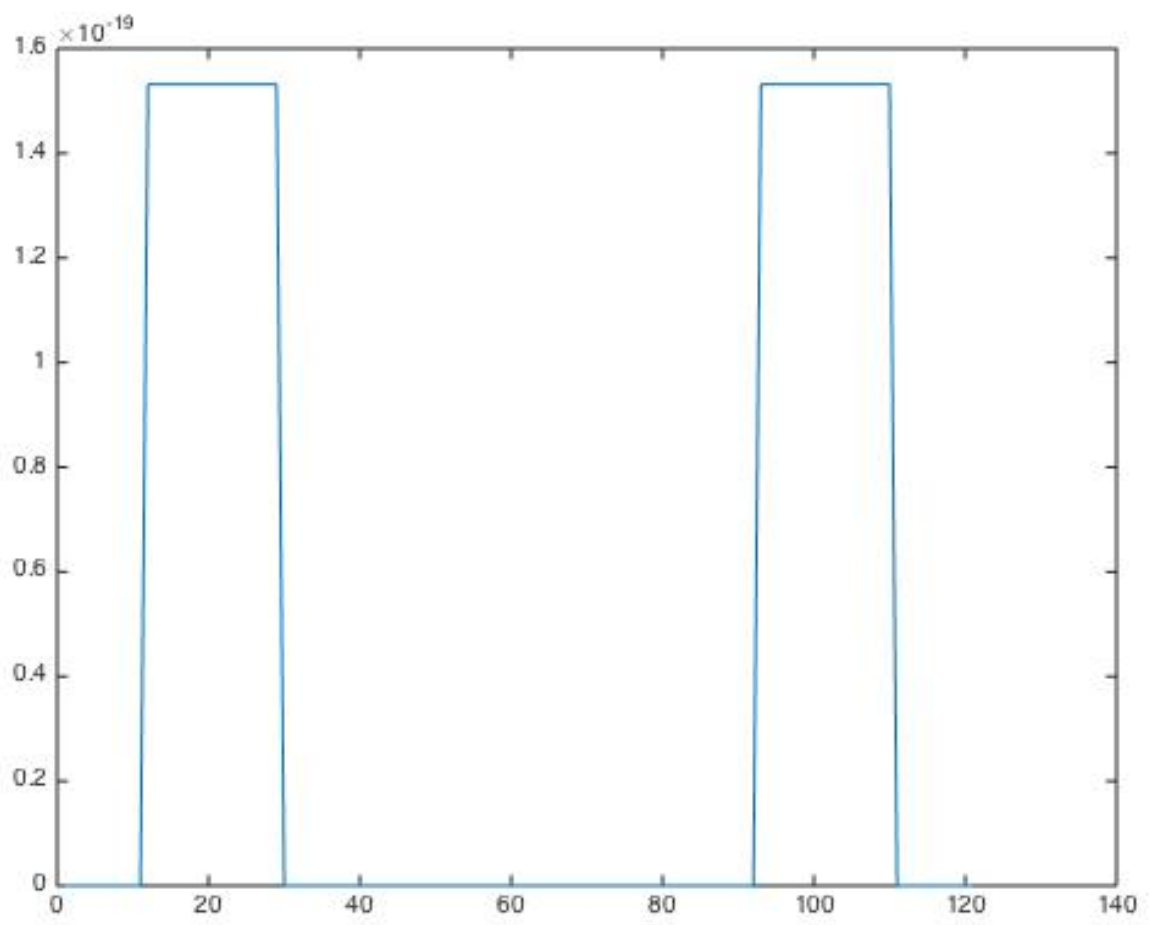

Figure 2.23

In the simulation the step number $\mathrm{N}=100$, the result of incident energy vs transmission probability is shown below: 


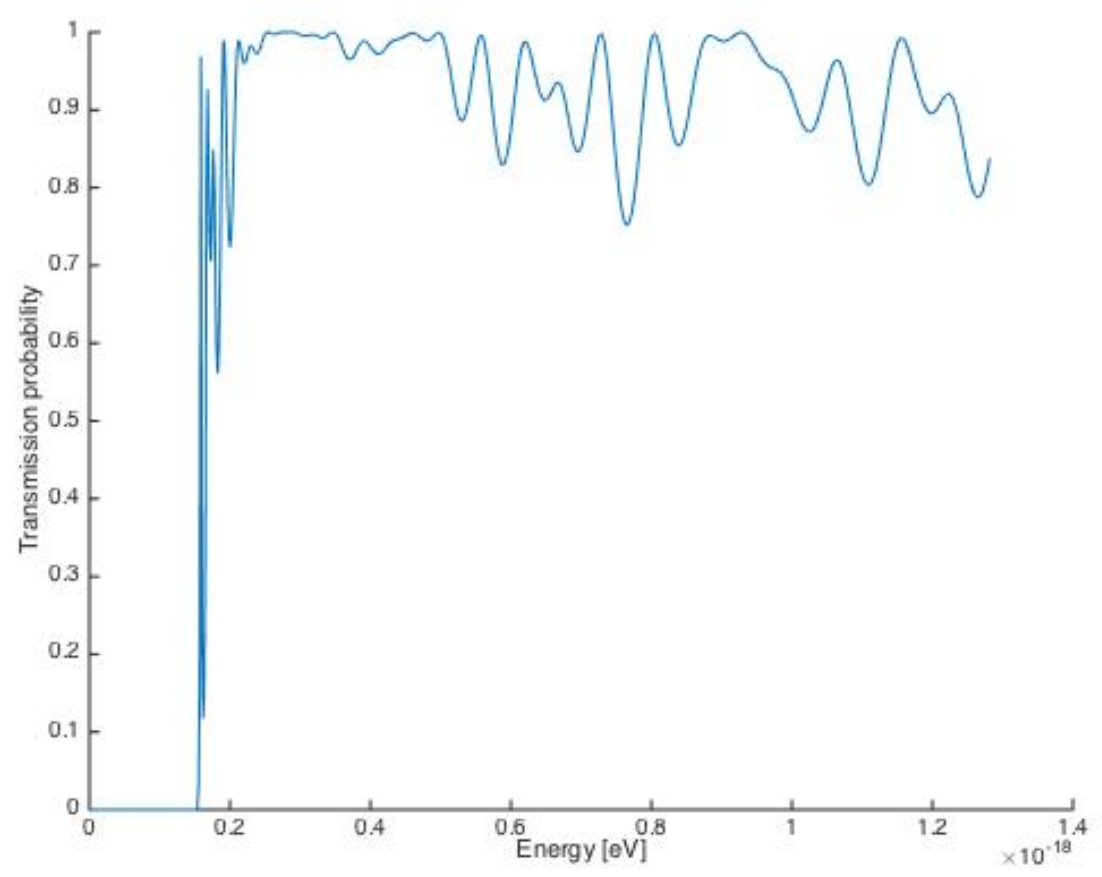

Figure 2.24

It quite different from single rectangular barrier. It shows the effect of effective mass. For a rectangular well that the effective mass varies with distance, the effective mass must be taken into consider while calculating the transmission coefficient. Here use formula (2.11) instead of $\left(2.11^{*}\right)$ so the probability would be

$$
D\left(E_{x}, V_{b}\right)=\frac{m_{N+1}}{m_{0}} \frac{k_{0}}{k_{N+1}}\left|\frac{1}{M_{22}}\right|^{2}
$$

Compare these three type of barrier, it decays that the barrier that contains more variables is more suitable to multistep method.

\subsection{Methods comparison}

For the complexity, when dealing with a simple rectangular barrier, the theoretical calculation is the easiest one, then comes the propagation matrix, multistep is the most complex one because it's necessary to calculate the matrices related with step number $\mathrm{N}$ to get the transmission amplitude. 
However while deal with parabolic barrier, the multistep method is the best choice. theoretical calculation is invalid and propagation matrix need the help of multistep and the calculation of matrices is much more complex.

Here a rectangular barriers is used to test the difference between acurancy of multistep, propagation matrix and theoretical calculation. The barrier width is $10 \mathrm{~nm}(100 \mathrm{Ai})$ and height is $1 \mathrm{ev}$. The step number $\mathrm{N}$ is 100.

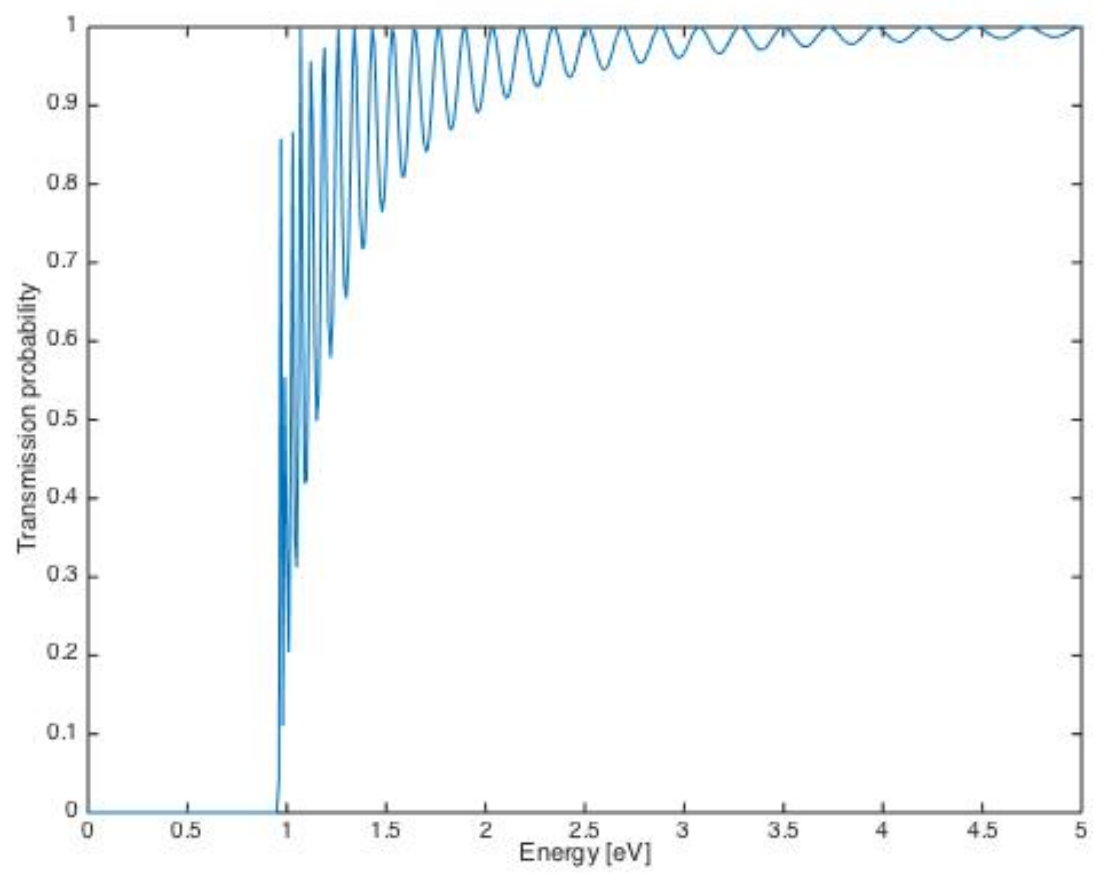

Figure 2.25 multistep 


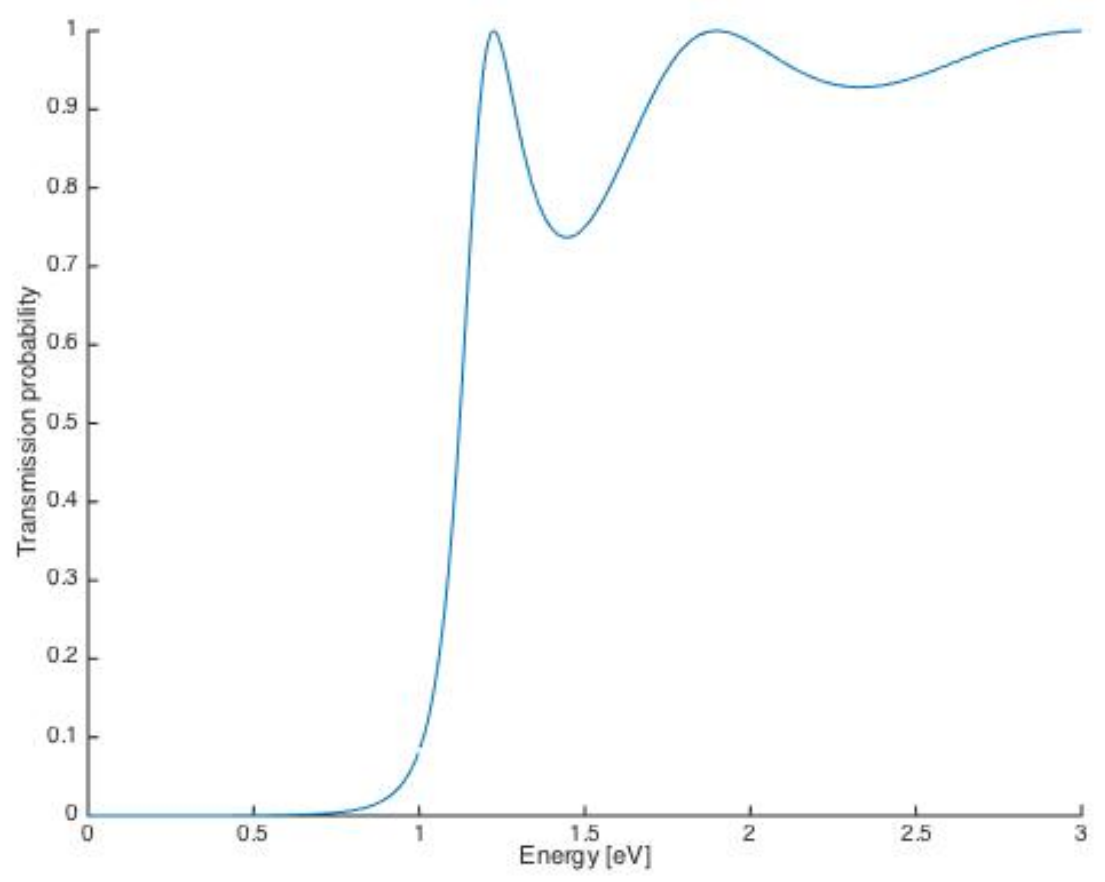

Figure 2.26 propagation matrix

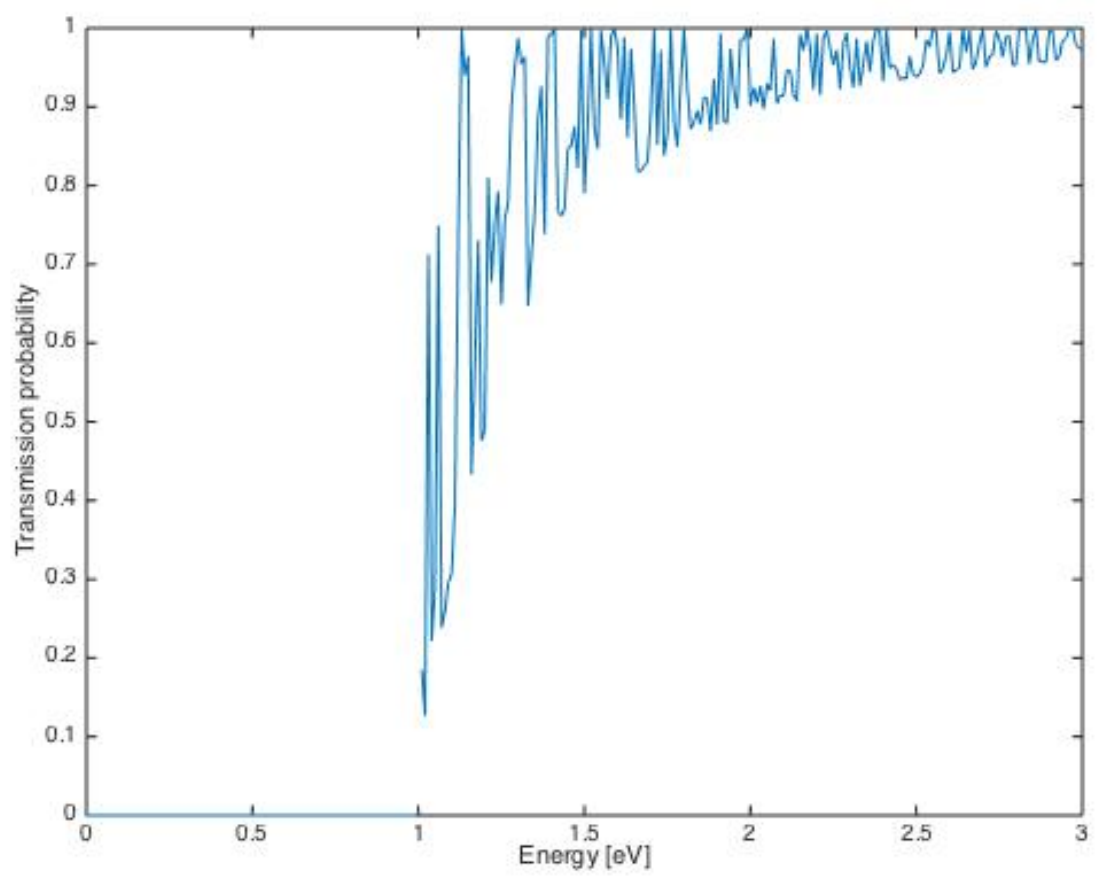

Figure 2.27 theoritical calculation

From figure 2.25,2.26 and 2.27, the conclusion could be drawn that the method really has influence on result accuracy even with all the same variable. The highest accuracy belongs to multistep. 


\subsection{Possible Further development}

Although the multistep method is better compared to other ones, it still contains disadvantages. The complexity makes it nearly impossible to use it by hand. So in the future developments may be achieved in reducing the complexity meanwhile keeping the accuracy of this method. Besides the phenomenon that the probability large than 1 happened in parabolic barrier is a worthy to put further concentration on. 


\subsection{Reference}

[1] M. Razavy, Quantum theory of tunneling. River Edge, NJ: World Scientific, 2003.

[2] E. Schrödinger, An Undulatory Theory of the Mechanics of Atoms and Molecules. Physical Review 28 (6): 1049-1070, 1926

[3] D. Griffiths, Introduction to quantum mechanics. Upper Saddle River, NJ: Pearson Prentice Hall, 2005.

[4][5][7] Y. Ando and T. Itoh, 'Calculation of transmission tunneling current across arbitrary potential barriers', J. Appl. Phys., vol. 61, no. 4, p. 1497, 1987.

[6] P. Turley and S. Teitsworth, 'Electronic wave functions and electron-confined-phonon matrix elements in GaAs/ Al x Ga 1 - x As double-barrier resonant-tunneling structures', Phys. Rev. B, vol. 44, no. 7, pp. 3199-3210, 1991.

[8] C. Chen, Introduction to scanning tunneling microscopy. New York: Oxford University Press, 1993.

[9] N. Yang, W. Henson, J. Hauser and J. Wortman, 'Modeling study of ultrathin gate oxides using direct tunneling current and capacitance-voltage measurements in MOS devices', IEEE Trans. Electron Devices, vol. 46, no. 7, pp. 1464-1471, 1999.

[10] L. Li, 'Electromechanical resistive switching via back-to-back Schottky junctions', AIP Advances, vol. 5, no. 9, p. 097138, 2015.

[11] A. Wacker, 'Semiconductor superlattices: a model system for nonlinear transport', Physics Reports, vol. 357, no. 1, pp. 1-111, 2002.

[12] A. Levi and T. Chiu, 'Room-temperature operation of hot-electron transistors', Appl. Phys. Lett., vol. 51, no. 13, p. 984, 1987. 


\section{Chapter3: Study on thermoelectric performance of twisted bilayer graphene nanoribbons junction by using first principle theory}

We investigate the electron transport and thermoelectric property of twisted bilayer graphene nanoribbon junction (TBGNRJ) in $0^{\circ}, 21.8^{\circ}, 38.2^{\circ}$ and $60^{\circ}$ rotation angles by first principles calculation with Landauer-Buttiker and Boltzmann theories. It is found that TBGNRJs exhibit a strong reduction of thermal conductance compared with the single graphene nanoribbon (GNR) and negative differential resistance (NDR) in $21.8^{\circ}$ and $38.2^{\circ}$ rotation angles under $\pm 0.2 V$ bias voltage. More importantly, three peak $Z T$ values of $2.0,2.7$ and 6.1 can be achieved in the $21.8^{\circ}$ rotation angle at $300 \mathrm{~K}$. The outstanding $Z T$ values of TBGNRJs are interpreted as the combination of the reduced thermal conductivity and enhanced electrical conductivity at optimized angles.

\subsection{Introduction}

Graphene is the first true 2-dimensional (2D) material, which consists of carbon atoms forming regular hexagonal lattice[1]. In the prior research, many outstanding properties of graphene were discovered, such as high electrical conductivity and carrier mobility[2-6], high thermal conductivity[7], and superior mechanical properties[8, 9]. Hence graphene can have many applications in electronic and mechanical devices. In particular, it has been applied in thermoelectric devices[10, 11]. The energy conversion efficiency of thermoelectric materials can be described by the figure of merit $Z T=$ 
$S^{2} G_{e} T /\left(\kappa_{e}+\kappa_{p h}\right)$, where the $G_{e}, S$ and $T$ are the electrical conductance, Seebeck coefficient and temperature, respectively. $\kappa_{e}$ and $\kappa_{p h}$ are the heat transport coefficient of electrons and phonons. A good thermoelectric material should have a high electrical conductivity, Seebeck coefficient and low thermal conductivity. On one hand, the Dirac-cone band structure of graphene makes it to display a high electrical conductivity $[5,6]$. On the other hand, the thermoelectric performance of graphene is poor because of its high thermal conductivity, and the closed bandgap which leads to a small Seebeck coefficient[12]. As a result, the key to improve the $Z T$ of graphene devices is find a trade-off between the Seebeck coefficient, electrical conductance and heat transport coefficient. In prior studies, the specially designed nanostructured graphene can have increased Seebeck coefficient and suppressed heat transport coefficient without greatly reducing electrical conductance due to the quantum confinement effect[10, 13, 14]. In these graphene devices, graphene nanoribbons (GNRs) has demonstrated better thermoelectric performances as the Seebeck coefficient and $Z T$ value can be increased by the finite size effect[15-18]. One of earlier attempts on the thermoelectric performance of GNRs was reported by Ouyang et al[18]. They found that a higher Seebeck coefficient in GNRs compared with the pristine graphene is attributed to the edge geometry of GNRs, which plays an important role in enhancing the thermoelectric performance. In 2012, Jin et al reported that the armchair GNR exhibits a higher $Z T$ value than zigzag GNRs, and $Z T$ value increases with the decrease of the GNRs width[15]. A chevron type edge GNR was proposed demonstrating a $Z T$ value of 3.25 at $800 \mathrm{~K}[19]$. Nguyen et al discovered that the thermal conductance in the bilayer GNRs is hundreds times weaker than the pristine graphene because the weak van der Waals ( $v d W$ ) interaction between two layers[20]. In recent several years, in order to improve thermoelectric performance of GNRs, different schemes based on more sophisticated GNR nanostructures have been designed [21, 22], such as using boundary effect[16, 23, 24], interface effect[25], doping[17, 26], and structure 
defect or wrinkles[27-30].

Bilayer graphene structures with optimized twisting angles aiming to achieve much improved performances such as high temperature superconductivity and strong interlayer coupling have been theoretically and experimentally investigated recently [31-34]. Because the bilayer graphene structure efficiently limits the thermal conductivity in the normal direction to the 2D plane attributed to the vdW force[20], this will lead to a new approach to further increase the thermoelectric performance.

In this work, the electron transport and thermoelectric performance of twisted bilayer graphene nanoribbon junction (TBGNRJs) in $0^{\circ}, 21.8^{\circ}, 38.2^{\circ}$ and $60^{\circ}$ rotation angles are systematically researched using the first principles method, which has been applied in previous studies on GNR junctions[20, 35]. It is discovered that that TBGNRJs exhibit negative differential resistance (NDR) in $21.8^{\circ}$ and $38.2^{\circ}$ rotation angles under $\pm 0.2 \mathrm{~V}$ bias voltage. Moreover, $Z T$ values of 2.0, 2.7 and 6.1 can be achieved at different chemical potentials for the $21.8^{\circ}$ rotation angle at $300 \mathrm{~K}$.

\subsection{Computational procedure}

The theoretical simulation of the twisted bilayer graphene structures starts with the Generalized Lattice Match (GLM) method, which is for investigating the relationship between the mismatch strain and the number of atoms[36]. The optimization of twisting angles is conducted by using the balanced mismatch strain and number of atoms, it is targeted to have a lower mismatch strain and at the same time a smaller number of atoms. As shown in the Figure 3.1(a) and (b), the vectors $\mathbf{v}_{1}$ and $\mathbf{v}_{2}$ defined the surface cell of the bottom graphene layer while the vectors $\mathbf{u}_{1}$ and $\mathbf{u}_{2}$ as the surface cell of the top graphene layer. The 
relationship between $\left[\mathbf{v}_{1}, \mathbf{v}_{2}\right]$ and $\left[\mathbf{u}_{1}, \mathbf{u}_{2}\right]$ is expressed as $A\left[\mathbf{u}_{1}, \mathbf{u}_{2}\right]=\left[\mathbf{v}_{1}, \mathbf{v}_{2}\right]$, where $\mathbf{A}$ is the affine transformation matrix. The rotation matrix $\mathbf{U}$ has a form as[36].

$$
\begin{array}{r}
\mathbf{U}=\left[\begin{array}{cc}
\cos (\phi) & -\sin (\phi) \\
\sin (\phi) & \cos (\phi)
\end{array}\right] \\
\phi=\left|\phi_{a}-\phi_{b}\right| / 2 \\
\mathbf{P}=\boldsymbol{U}^{\boldsymbol{T}} \mathbf{A}
\end{array}
$$

where, $\phi$ is the twisting angle of bilayers graphene, $\phi_{a}$ is the angle between the vectors $\mathbf{u}_{1}$ and $\mathbf{u}_{2}, \phi_{b}$ is the angle between the vectors $\mathbf{v}_{1}$ and $\mathbf{v}_{2}$, matrix $\mathbf{P}$ defines the 2D strain tensor for deforming one cell into the other. We only calculate the twisting angles from $0^{\circ}$ to $60^{\circ}$ because the crystal structure of graphene is a regular hexagon. In order to sustain the structural stability and avoid having a large size cell, we analyzed the twisting angles of $0^{\circ}, 21.8^{\circ}$, $38.2^{\circ}$ and $60^{\circ}$, at which the strain of lattice mismatch is calculated to be $0 \%$. Previous studies have shown that $A A\left(0^{\circ}\right.$ rotation angle) and $A B\left(60^{\circ}\right.$ rotation angle) stacking of bilayer graphene are the most common stacking style in the research [37]. The other twisting angles (21.8 ${ }^{\circ}$ and $\left.38.2^{\circ}\right)$ were chosen, because they had a small size of cell comparing with other rotation angles. 
(a)

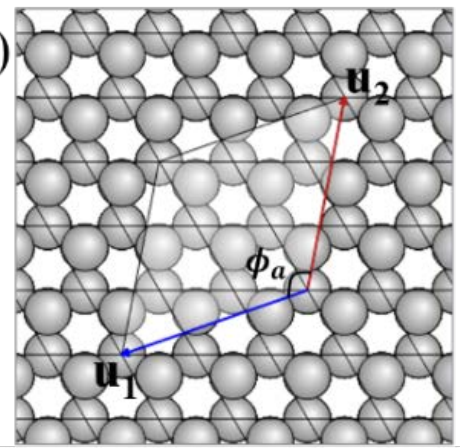

(c)

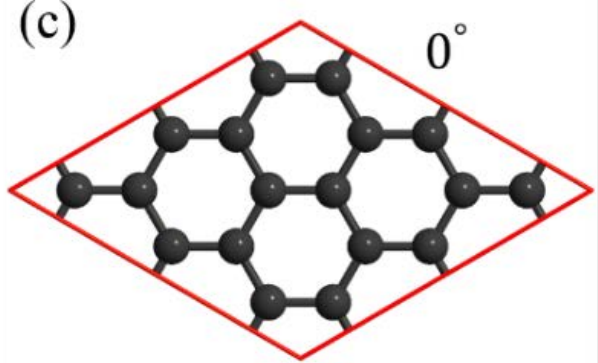

(e)

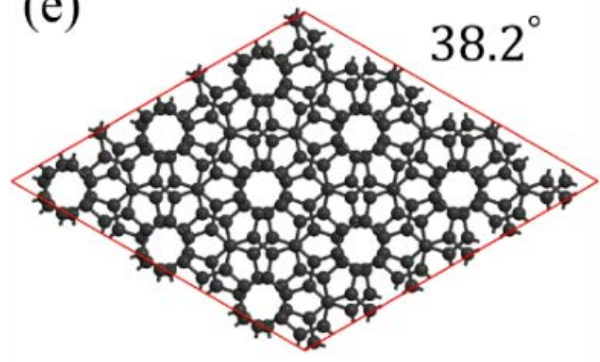

(b)

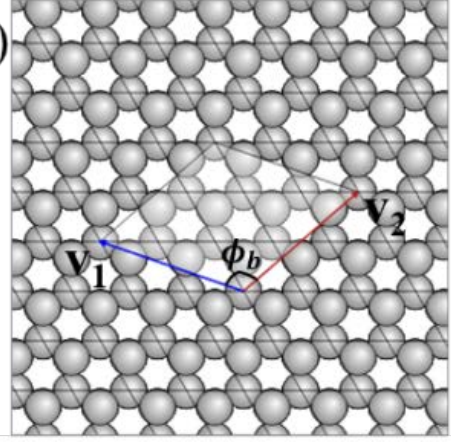

(d)

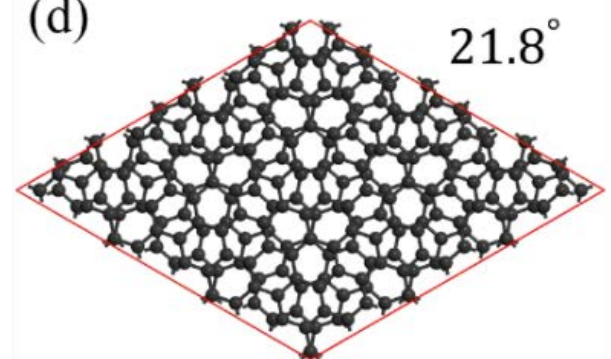

(f)

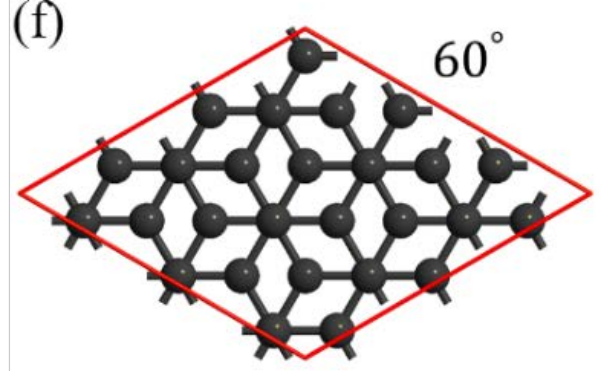

Figure 3.1. The surface cell of top (a) and bottom of graphene (b). (c)-(f) show the top view of the atomic arrangement of the twisted bilayer graphene with $0^{\circ}, 21.8^{\circ}, 38.2^{\circ}$ and $60^{\circ}$ rotation angles, respectively.

All the modelling and calculations have been implemented by the Quantum Atomistix ToolKit (ATK2018) simulation tools[38]. Calculations of the electronic properties have been performed within the framework of density functional theory (DFT). In the geometry optimization, we use the semi-empirical corrections by the Grimme DFT-D2 model to calculate the distance between two layers, which accounts the long-range vdW interaction [39]. The structure is fully relaxed until the force on each atom becomes smaller than $0.01 \mathrm{eV} / \AA$. The generalized gradient approximation (GGA) with the parametrization of Perdew-Burke-Ernzerhof (PBE), cut-off energy of 150 Ry and $12 \times 12 \times 1$ k-points 
grid were used. To avoid the interaction of the periodic boundary conditions, a large vacuum spacing of at least $25 \AA$ is added along the normal direction to the electrons transport plane. In our transmission calculation, the device model is divided into central part, left and right electrodes. The Brillouin zone of the junction is sampled by a $1 \times 1 \times 100 k$-mesh and a double-zeta polarized for all atoms. The electronic and phonon transmissions are calculated by the nonequilibrium Green function method and molecular dynamics method, respectively. The calculated rotation angle $(\theta)$, lattice constant $(a)$, mean absolute strain $(\varepsilon)$ and surface distance between two layers $(d)$ are listed in Table 1. For $A A\left(0^{\circ}\right.$ rotation angle) and $A B\left(60^{\circ}\right.$ rotation angle) stacking structures of bilayer graphene, the interface distance is $3.35 \AA$, which is close to those of the same bilayer graphene structures in references[40, 41]. As shown in Figure 3.2, the fat band structures and projected shells for four twisted bilayer graphene cells have been calculated along the path through $\Gamma-M-K-\Gamma$. The energy bands originated from the $s, p$ and $d$ orbitals are marked by red, blue and green lines, respectively. We obtained two Dirac cones around the $K$ point originated from $p$ orbital because the band splitting occurs when two graphene layers interact. The splitting between the bands in the Dirac cones is responsible for low-dispersion bands near the $M$ point[41, 42]. Although the top views of the atomic arrangement of the twisted bilayer graphene cells (Figure 3.1) look different, the band structures calculated for the rotation angles $0^{\circ}$ and $60^{\circ}$ seem very similar, the same for the rotation angles $21.8^{\circ}$ and $38.2^{\circ}$. It is because that the crystal structure of graphene is a regular hexagon, which has rotational symmetry. The $0^{\circ}$ and $60^{\circ}\left(21.8^{\circ}\right.$ and $\left.38.2^{\circ}\right)$ are two sets of rotational symmetry angles. The similarity of band structures with rotational symmetry angles were described in reference [43].

Table 3.1. Rotation angle $(\theta)$, lattice constant (a), mean absolute strain $(\varepsilon)$, and surface distance between two layers (d) for bilayer graphene. 


\begin{tabular}{llll}
\hline$\theta\left(^{\circ}\right)$ & $a(\AA)$ & $\varepsilon$ & $d(\AA)$ \\
\hline 0 & 2.46 & $0 \%$ & 3.35 \\
21.8 & 6.51 & $0 \%$ & 3.25 \\
38.2 & 6.51 & $0 \%$ & 3.25 \\
60 & 2.46 & $0 \%$ & 3.35 \\
\hline
\end{tabular}
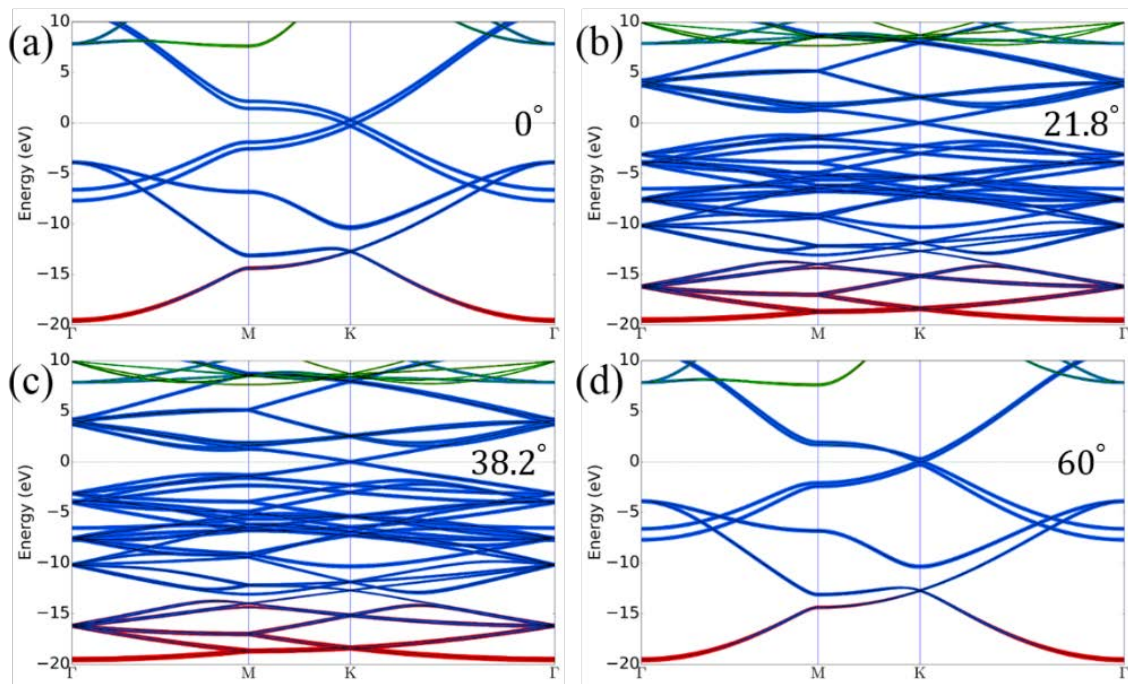

Figure 3.2. The computed fat band structures and projected shells of bilayer graphene structures with $0^{\circ}$ (a) $21.8^{\circ}\left(\right.$ b), $38.2^{\circ}$ (c) and $60^{\circ}$ (d) rotation angles.

Based on these twisted bilayer graphene structures, overlapped TBGNRJs has been devised, followed by the investigation on electron transport and thermoelectric performance. Figure 3.3 shows the details of the device model, which is divided into central scattering region (SR), left electrode (LE) and right electrode (RE). The length of the overlap region in the TBGNRJs is 5 unit-cells along the transport direction, which is the minimum length that avoids modelling imperfectness, i.e. length shorter than 5 unit-cells will result in overlapped part entering to the electrode extension region. With the increase of the overlap length, the interlayer coupling enhances, which leads to increase of thermal conductance and reduction of $Z T$, which is consistent with the prior reference [20]. Moreover, models with longer overlap regions take much longer time to compute. 
(a)

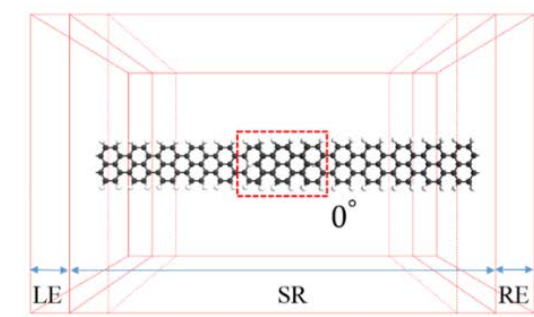

(c)

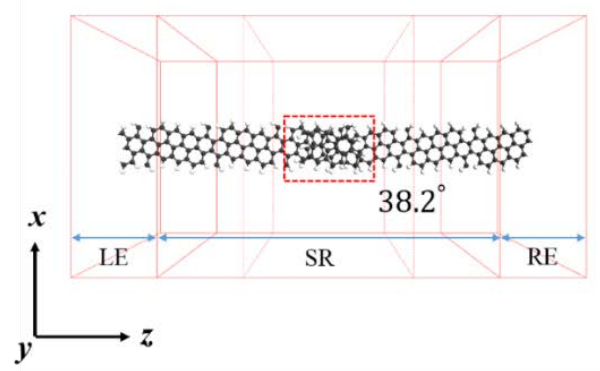

(b)

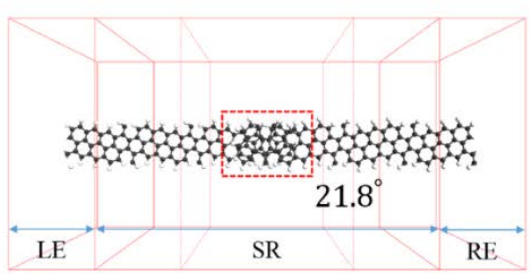

(d)

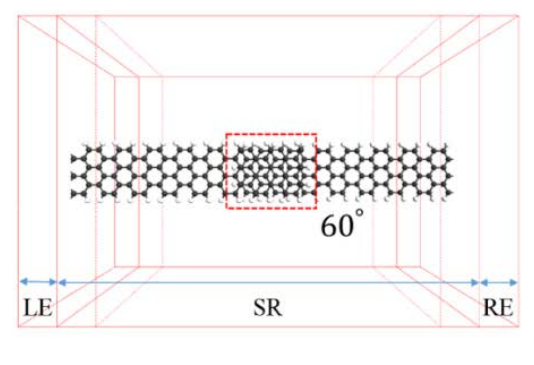

Figure 3.3. The top view of the $0^{\circ}$ (a) $21.8^{\circ}$ (b), $38.2^{\circ}$ (c) and $60^{\circ}$ (d) TBGNRJs.

\subsection{Results and discussion}

First, we investigate the $I-V$ characteristics of these TBGNRs. The current across a TBGNR can be calculated from the Landauer-Buttiker equation[44]:

$$
I=\frac{e}{h} \int d E\left(f_{L}(E)-f_{R}(E)\right) T_{e}(E)(3.4)
$$

Where, $T_{e}(E)=\operatorname{Tr}\left[t^{+} t\right](E)$ is the total electron transmission, $f_{R}(E)$ and $f_{L}(E)$ are the Fermi distribution functions of the right and left electrode, respectively.

Figure 3.4(a) shows the calculated current as a function of the bias voltage for the TBGNRJs in $0^{\circ}, 21.8^{\circ}, 38.2^{\circ}$ and $60^{\circ}$ rotation angles. With the increase of the bias voltage, the current displays an increasing trend. Moreover, the I-V curves of $0^{\circ}$ exhibit a behavior similar to a back-to-back p-n junction. However, it is not the case for the $60^{\circ}$ TBGNRJ where the I-V curve exhibits transistorlike behavior. Similar results were shown in a prior publication[40]. We calculated the $I-V$ curves with different widths for $0^{\circ}$ and $60^{\circ}$ TBGNRJs in 
Figure 3.4(c) and (d). For two graphene cells width, the $I-V$ curves of $0^{\circ}$ and 60 - TBGNRJs show back-to-back p-n junction and transistor behavior, respectively. However, with the increase of the width, the shape of $I-V$ curves changed, which means that the electronic transport properties of graphene nanoribbons strongly depend on their width [45]. Because of connecting only the top layer to the left electrode and bottom layer to the right electrode, the current flowing through the interface depends on the coupling between bilayers. For $0^{\circ}$ and $60^{\circ}$ rotation angles, different interlayer angles induce different interlayer coupling and electronic transport, which causes various development trends of $I-V$ curves. At $21.8^{\circ}$ and $38.2^{\circ}$ rotation angles, the $I-V$ and $d I I d V$ curves (Figure $3.4(b)$ ) are asymmetric, which is due to the non-central symmetric structure in the twisted junctions. Under the $1 \mathrm{~V}$ bias voltage, the current intensity of $21.8^{\circ}$ TBGNRJ is higher than $38.2^{\circ}$ TBGNRJ, while this relationship reverses at the $-1 \mathrm{~V}$ bias voltage. Under the $0^{\circ}$ and $60^{\circ}$ rotation angles, the $I-V$ and $d I I d V$ curves exhibit symmetric behavior. For the $I-V$ curve, under the same magnitude of the bias voltage, the current intensity of $0^{\circ}$ TBGNRJ is smaller than $60^{\circ}$ TBGNRJ for all voltages. For the dlldV curves, the value of $60^{\circ}$ TBGNRJ is higher than $0^{\circ}$ TBGNRJ from $-0.6 V$ to $0.6 V$. However, $60^{\circ} \mathrm{TBGNRJ}$ is lower than $0^{\circ} \mathrm{TBGNRJ}$ at the rest voltages. More interestingly, the $I-V$ and $d I I d V$ curves demonstrate NDR in $21.8^{\circ}$ and $38.2^{\circ}$ rotation angles under $\pm 0.2 \quad V$ bias voltage, which is mainly caused by transmission spectra under different bias voltages [46-48]. Although the calculated NDR is rather small, the NDR effect linked with the bias voltage was proposed in a prior research [49]. The physical reason of the NDR can be explained by analyzing the relationship between the transmission spectra and the applied bias voltage. Taking the $21.8^{\circ}$ rotation angle as an example, the transmission spectra are displayed for the bias voltages of $0.2 \mathrm{~V}, 0.4 \mathrm{~V}, 0.6 \mathrm{~V}$, $0.8 \mathrm{~V}$ and $1.0 \mathrm{~V}$ in the Figure 3.4(e). Under the $0.2 \mathrm{~V}$ bias voltage, the bias window directly related to the bias voltage is defined by $\pm 0.1 \mathrm{eV}$ around the Fermi level. The current rises to about $1.7 \mu \mathrm{A}$, which is caused by a peak of 
transmission spectra within the bias window. At the bias voltages of $0.4 \mathrm{~V}$ and $0.6 \mathrm{~V}$, although the bias window widens, the peak values of transmission spectra within the bias window decrease, which indicates a reduced electron transmission. This reflects to a clear reduction of the current from about $1.7 \mu \mathrm{A}$ at $0.2 \mathrm{~V}$ to $0.07 \mu \mathrm{A}$ at $0.6 \mathrm{~V}$ and results to a peak-to-valley ratio around 24.3. However, with the bias voltage higher than $0.6 \mathrm{~V}$, the peak of transmission spectra within the bias window increases, and the current increases subsequently.

(a)

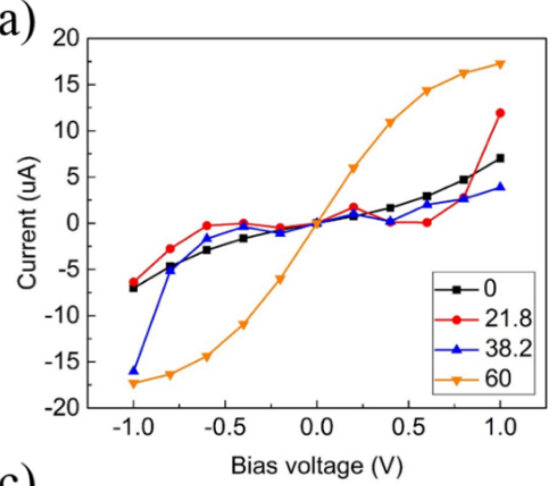

(c)

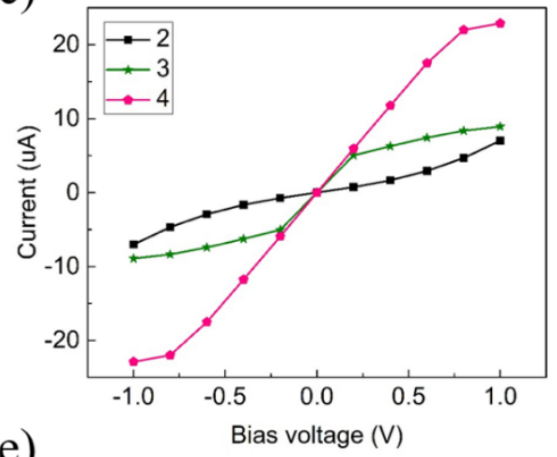

(b)

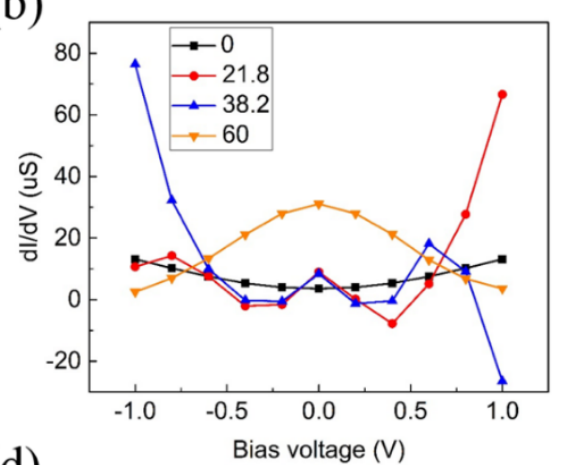

(d)

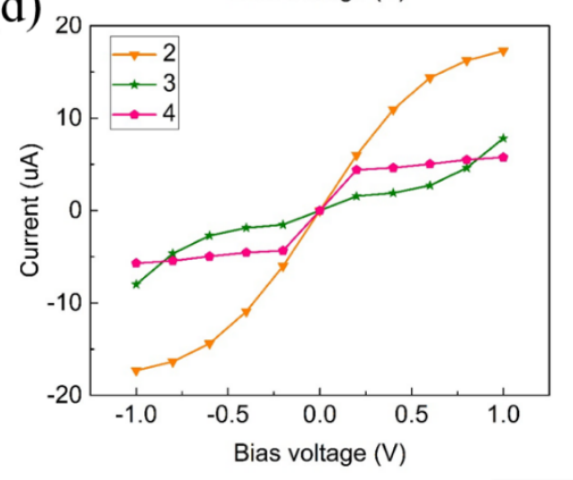

(e)

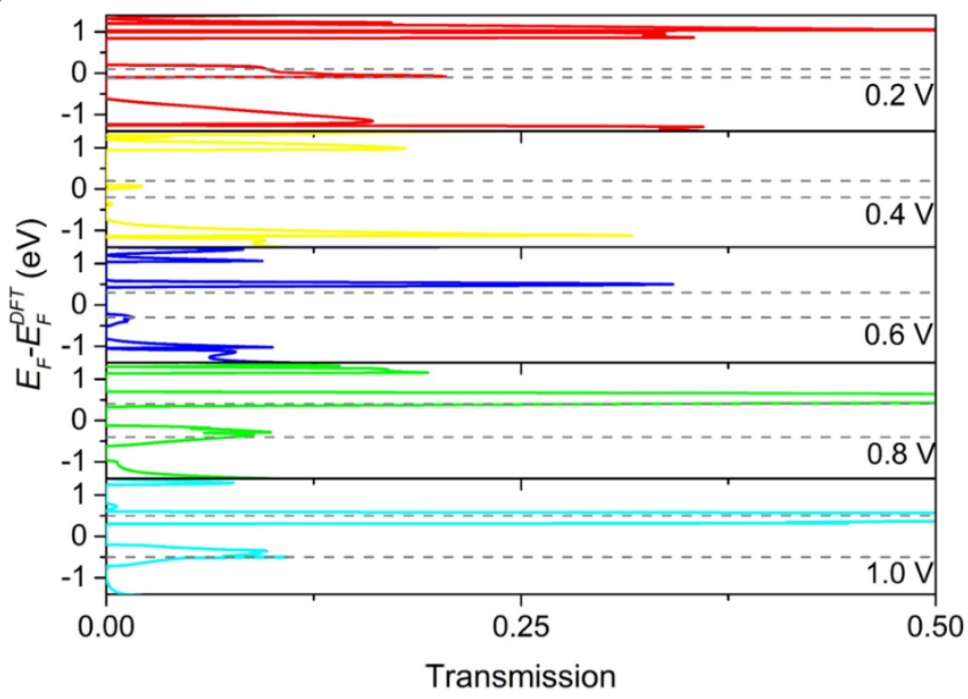


Figure 3.4. The I-V (a) and dIIdV (b) curves of the TBGNRJs with $0^{\circ}, 21.8^{\circ}, 38.2^{\circ}$ and $60^{\circ}$ rotation angles. (c) and (d) show the $\mathrm{I}-\mathrm{V}$ with $0^{\circ}$ and $60^{\circ} \mathrm{TBGNRJs}$ for 2, 3 and 4 graphene cells widths. (e) The electron transmission spectra of $21.8^{\circ}$ TBGNRJ under $0.2 \mathrm{~V}, 0.4 \mathrm{~V}, 0.6$ $V, 0.8 \mathrm{~V}$ and $1.0 \mathrm{~V}$ bias voltages.

Thermoelectric properties of the TBGNRJs for the above twisting angles have also been simulated. Microscopically, the electron transport properties of the TBGNRJs can be described by the Boltzmann equation with the Fermi-Dirac distribution $f_{F D}(E, T)$. The electron conductance, Seebeck coefficient and heat transport coefficient of electrons are given by[50]:

$$
\begin{gathered}
G_{e}(T)=\frac{e^{2}}{h} L_{0}(3.5) \\
\mathrm{S}(\mathrm{T})=\frac{1}{e T} \frac{L_{1}}{L_{0}}(3.6) \\
\kappa_{e}(T)=\frac{L_{0} L_{2}-L_{1}^{2}}{h t L_{0}}(3.7)
\end{gathered}
$$

Where, $e$ and $h$ are the electron charge and Planck's constant.

$$
L_{n}(T)=\int_{-\infty}^{+\infty}\left(E-E_{F}\right)^{n} T_{e}(E)\left(-\frac{\partial f_{F D}(E, T)}{\partial E}\right) d E(3.8)
$$

The heat transport coefficient of phonons is given by:

$$
\kappa_{p h}(T)=\frac{1}{2 \pi} \int_{0}^{\infty} \hbar w T_{p h}(\omega) \frac{\partial f_{B E}(\omega, T)}{\partial T} d \omega(3.9)
$$

Where, $f_{B E}(\omega, T)$ and $T_{p h}(\omega)$ are the Bose-Einstein distribution function and phonon transmission coefficient.

As shown in Figure 3.5, the electrical conductance, Seebeck coefficient, thermal conductance and $Z T$ obtained in the TBGNRJs in the four twisting angles at $300 \mathrm{~K}$ are displayed. In the results, the chemical potential is a difference between the Fermi energy $\left(E_{F}\right)$ and the DFT-predicted Fermi energy $\left(E_{F}^{D F T}\right)$. The valleys and peaks of electrical conductance are shown in Figure 3.5(a) because of different band structures and bandgaps of the monolayer and 
bilayer parts of the TBGNRJs[20]. In Figure 3.5(b), the development of thermal conductance at four rotation angles have a similar trend with electrical conductance, which is similar to what is reported in the reference[15]. This result illustrates that the Seebeck coefficient is a key parameter to achieve high $Z T$ value in the TGNRJs. In Figure 3.5(c), the maximum of Seebeck coefficient in $21.8^{\circ}$ and $38.2^{\circ}$ rotation angles is about $0.75 \mathrm{mV} / \mathrm{K}$, which is about 7.5 times higher than the pristine graphene $(\sim 0.1 \mathrm{mV} / \mathrm{K})[5,51]$. Figure $3.5(\mathrm{~d})$ shows the $Z T$ values of four TBNGRJs, four peak $Z T$ values are more than 1 for the 21.8 ${ }^{\circ}$ and $38.2^{\circ}$ rotation angles at $300 \mathrm{~K}$. Particularly, in the $21.8^{\circ}$ rotation angle, the maximum $Z T$ is 6.1 , which is higher than most reported $Z T$ values at room temperature[10, 52-54].

(a)

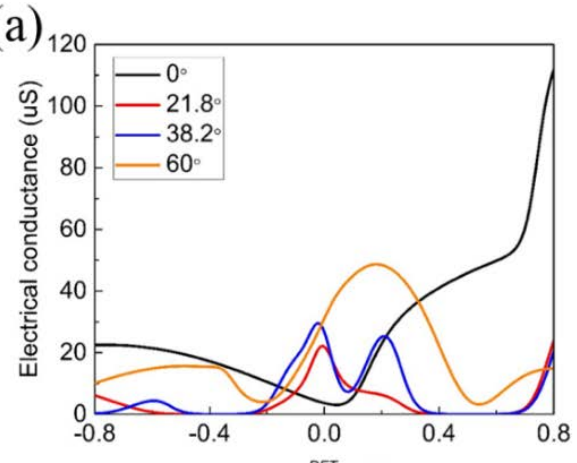

(c)

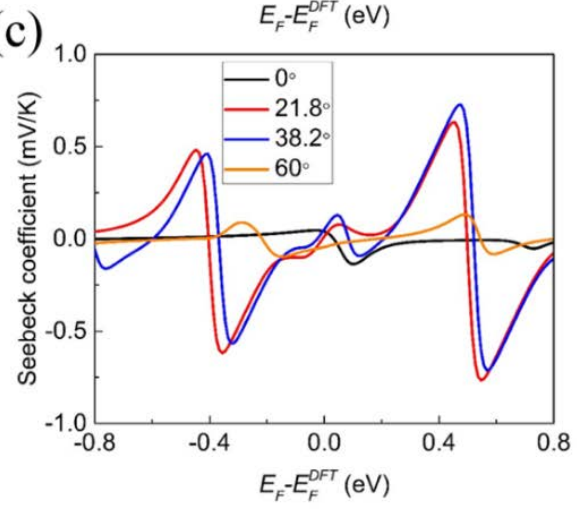

(b)

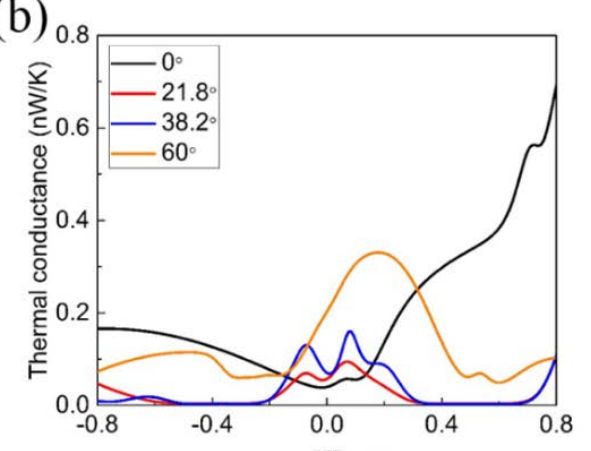

(d)

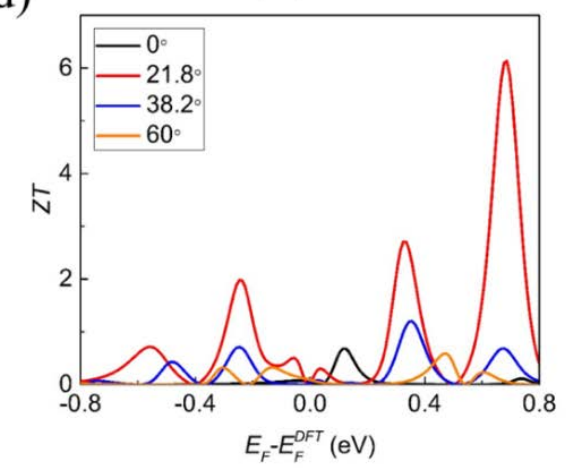

Figure 3.5. The electrical conductance (a), thermal conductance (b), Seebeck coefficient (c) and $Z T(d)$ obtained in the TBGNRJs with $0^{\circ}, 21.8^{\circ}, 38.2^{\circ}$ and $60^{\circ}$ rotation angles at $300 \mathrm{~K}$.

To summarize, band structures calculated in Figure 3.2 are from twisted bilayer graphene cells in Figure 3.1. Whereas the band structures in Figure 3.6 are for nanoribbon structures with hydrogen passivation shown in red dotted boxes in 
Figure 3.3. In our simulation, the twisted bilayer graphene nanoribbon is periodic along the $z$-direction. Comparing with the twisted bilayer graphene cells (Figure 3.1), all the carbon atoms at the edges of the twisted bilayer graphene nanoribbon are passivated by hydrogen atoms and a vacuum layer of $25 \AA$ is added along the $x$-direction. These modelling processes break the symmetry of original twisted bilayer graphene cells, thus inducing significant band structure differences between the angles $0^{\circ}$ and $60^{\circ}\left(21.8^{\circ}\right.$ and $\left.38.2^{\circ}\right)$. The band structures of the four bilayer graphene nanoribbons along the Brillouin path through $\Gamma-Z$ because the Seebeck coefficient is derived from the bandgap [55]. Bandgap opening is shown following the twisting of the bilayer graphene nanoribbons, which results in an increase of the Seebeck coefficient [56]. As shown in Figures 3.6(b) and (c), the bandgaps of the $21.8^{\circ}$ and $38.2^{\circ}$ TBGNRJs are $0.15 \mathrm{eV}$ and $0.14 \mathrm{eV}$, which are larger than the bandgaps of $0^{\circ}$ $(0.02 \mathrm{eV})$ and $60.0^{\circ}(0.11 \mathrm{eV})$ rotation angles. However, the bandgap opening of the $0^{\circ}$ TBGNRJ is lower than the $25 \mathrm{meV}$ (thermal fluctuation) at $300 \mathrm{~K}$, which is too small and tends to vanish in experiments.
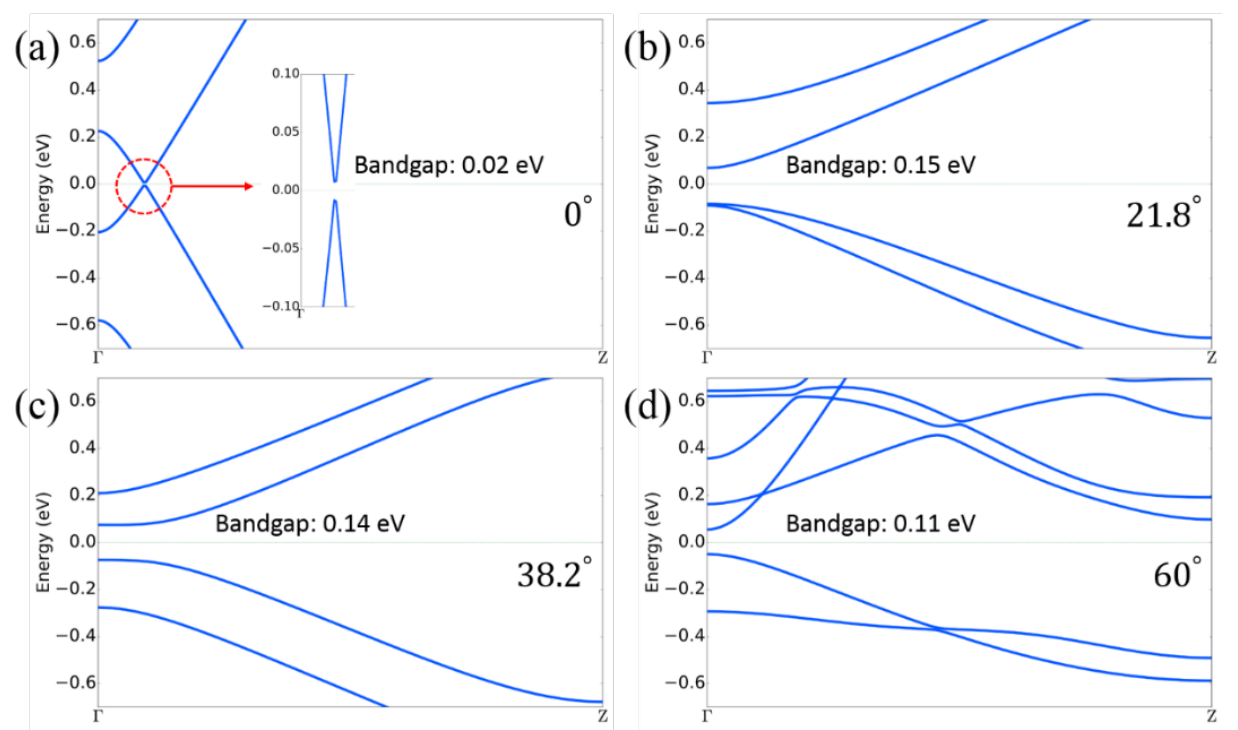

Figure 3.6. The computed band structures of the twisted bilayer graphene nanoribbons with 0 ${ }^{\circ}$ (a) $21.8^{\circ}\left(\right.$ b), $38.2^{\circ}$ (c) and $60^{\circ}$ (d) rotation angles. 
Figure 3.5 (a) and Figure 3.7 (a) clearly show that an electrical conductance and transmission spectra gap in the range $[0.3-0.7 \mathrm{eV}]$ observed for the $21.8^{\circ}$ and $38.2^{\circ}$ TBGNRJs, which is hypothesized to be the cause of a high Seebeck coefficient. In the Figure 3.5 (c) and Figure 3.7 (a), the position of the Seebeck coefficient peak is consistent with the position of the electrical conductance and transmission spectra gap (0.3 eV-0.7 $\mathrm{eV}$ ), which matches with the reference [57]. For $21.8^{\circ}$ and $38.2^{\circ}$ TBGNRJs, a clear transmission peak near the Fermi energy is due to the band bending and edge states, which has been described in prior theoretical and experimental research [58-60]. In the $21.8^{\circ}$ and $38.2^{\circ}$ TBGNRJs, a transmission spectra gap observed in the transmission spectra within the energy range from $0.3 \mathrm{eV}$ to $0.7 \mathrm{eV}$, which is not observed in the $0^{\circ}$ and $60^{\circ}$ TBGNRJs. It is known that the transmission spectra and electrical conductance gap can improve the Seebeck coefficient and thermoelectric performance [61, 62]. In our case, the transmission spectra and electrical conductance gaps are due to the special twisting angles between two graphene nanoribbons, which affect the electrons transport through the interface of the top and bottom of the bilayer. Because there is no such transmission spectra and electrical conductance gap with rotation angles $0^{\circ}$ and $60^{\circ}$, the Seebeck coefficient and $Z T$ remain very small in these structures. To summary, we analyzed the electrical conductance and thermal conductance for $21.8^{\circ}$ and $38.2^{\circ}$ TBGNRJs in a $0.2 \mathrm{eV}$ energy range consisting of the $Z T$ peak. As shown in Figure 3.7 (c) and (d), the main reason of the high $Z T$ is that at $21.8^{\circ}$ and $38.2^{\circ}$ angles, TBGNRJ exhibits a very small thermal conductivity $(K)$ with small variations along the energy axis, and a relatively large electric conductivity $\left(G_{e}\right)$. 
(a)

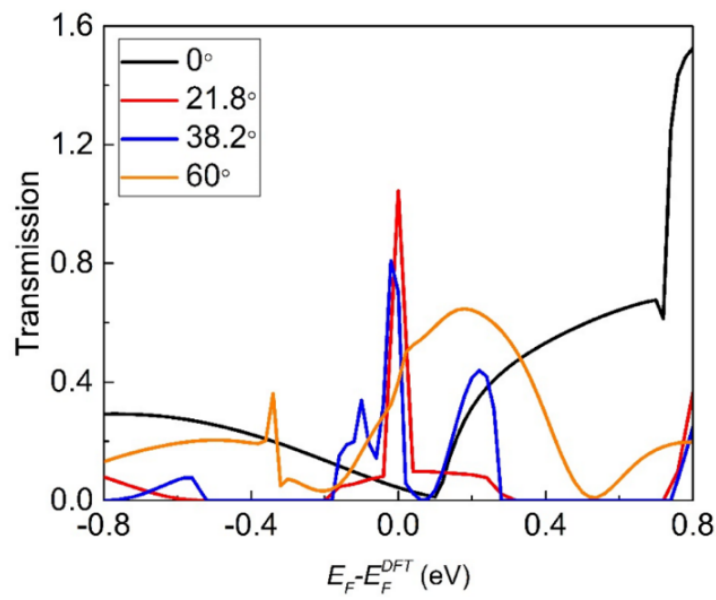

(b)

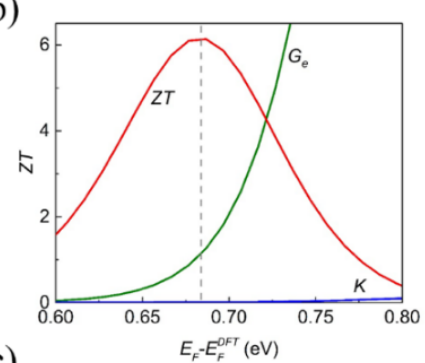

(c)

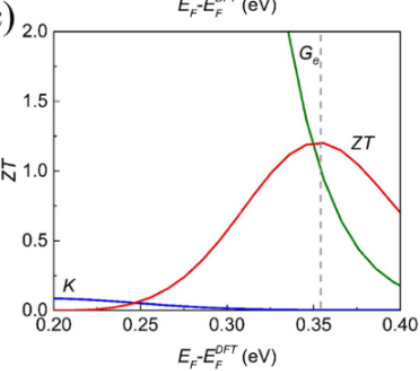

Figure 3.7. The electron transmission spectra obtained in the TBGNRJs at $300 \mathrm{~K}(\mathrm{a})$, the thermal conductance obtained in a single GNR of same width with the $0^{\circ} T B G N R J(b)$, electrical conductance, thermal conductance and $Z T$ in the $0.2 \mathrm{eV}$ energy range of the $Z T$ peak with $21.8^{\circ}$ (c) and $38.2^{\circ}$ (d) TBGNRJs.

Figure 3.8 shows a comparison of the thermal conductance of the TBGNRJs (shown in Figure 3.5b) to that of a single GNR of same width. It is shown that there is a strong reduction of thermal conductance for the TBGNRJs compared with the single GNR. It is concluded that one of important contributions to the $Z T$ enhancement of TBGNRJs should come from the strong reduction of thermal conductance. From Figure 3.5, the peaks of $Z T$ higher than 1 exist in $21.8^{\circ}$ and $38.2^{\circ}$ rotation angles. We shall compute the thermal conductance of electrons and phonons for these TBGNRJs. As shown in Figure 3.9(a), the phonon contribution to the overall thermal conductance is much more than the electron contribution for $Z T$ values of 2.7 and 2.0 in the $21.8^{\circ}$ TBGNRJs. However, at the $Z T$ value of 6.1 , the electron contribution to thermal conductance is comparable to that from the phonon. The maximum $Z T$ value of $38.2^{\circ}$ TBGNRJs is 1.2, at which thermal conductance of electrons is slightly larger than that of phonons (Figure 3.9(c)). Figure 3.9(b) and (d) show that the thermal conductance of phonons increases with the rise of temperature. With 
all the electron and phonon transport properties calculated, we can evaluate the figure of merit of TBGNRJs. In Figure 3.5(d), all the outlines of $Z T$ are rather asymmetric, and each of them has some peaks around the $0 \mathrm{eV}$. So, we can enhance $Z T$ by appropriate $n$-type or $p$-type doping in these TBGNRJs at 300 $\mathrm{K}$. For the $21.8^{\circ}$ rotation angles, there are three peak values of $Z T \sim 2.0,2.7$ and 6.1 at around $-0.25 \mathrm{eV}, 0.33 \mathrm{eV}$ and $0.68 \mathrm{eV}$, which means $n$-type doping are more favorable than $p$-type doping to improve the thermoelectric performance in the $21.8^{\circ}$ TBGNRJs. In the $38.2^{\circ}$ TBGNRJs, the maximum $Z T$ value is 1.2 at $0.33 \mathrm{eV}$, which can be obtained by $n$-type doping. All these results suggest that $21.8^{\circ}$ TBGNRJs can obtain a significant improvement in thermoelectric performance at $300 \mathrm{~K}$.

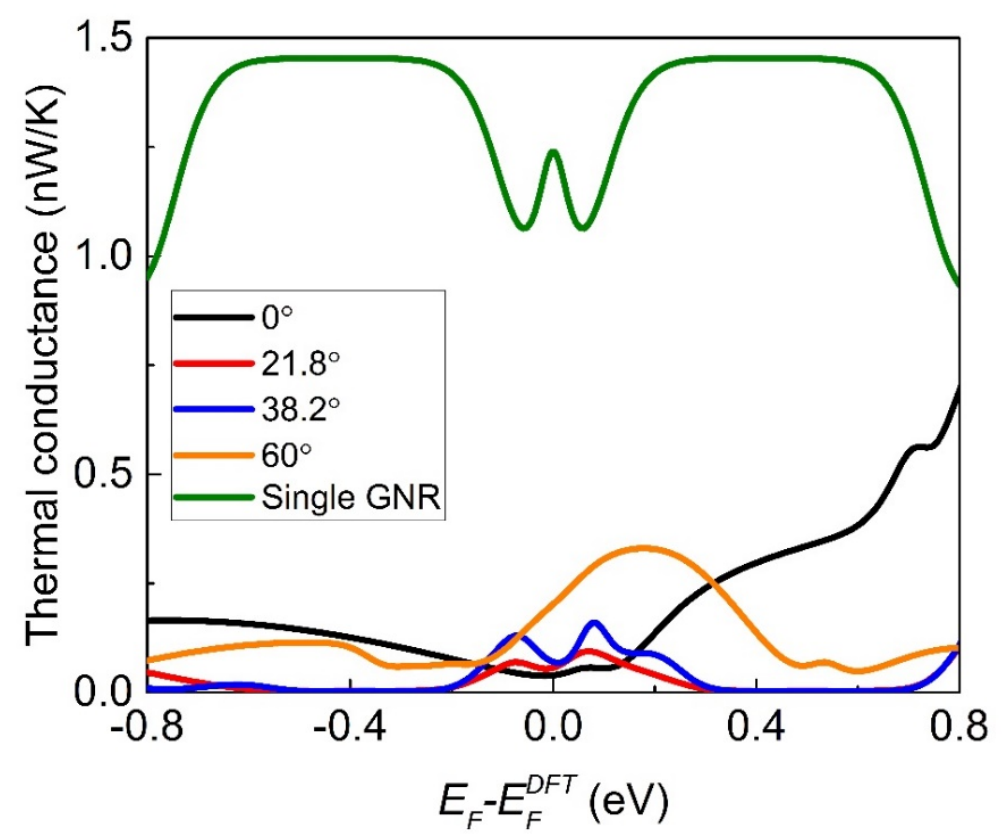

Figure 3.8. The thermal conductance obtained in a single GNR of same width with the TBGNRJs. 
(a)

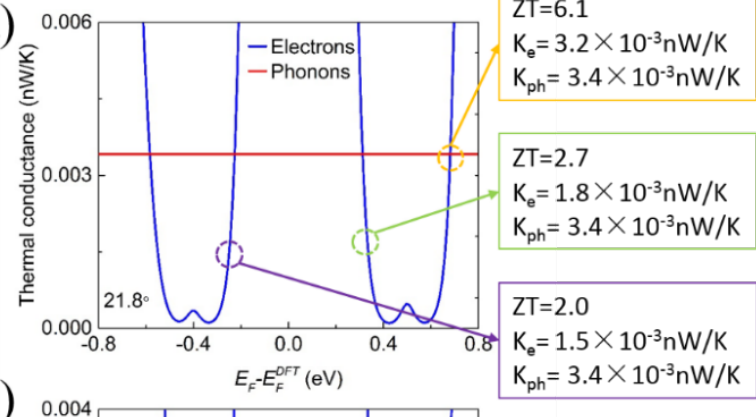

(c)

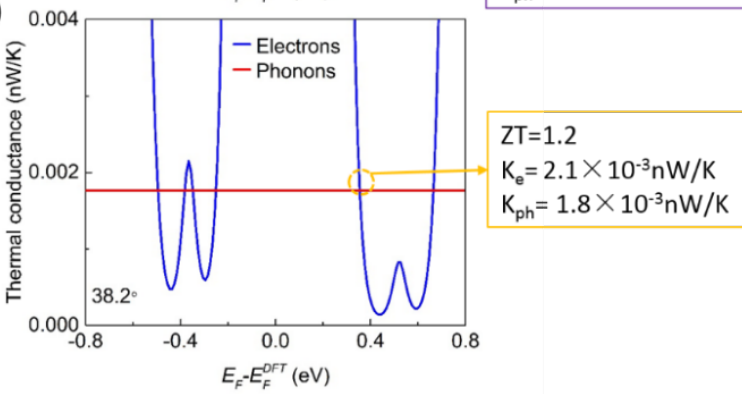

(b) $0 . 0 0 6 \longdiv { 2 1 . 8 ^ { \circ } }$

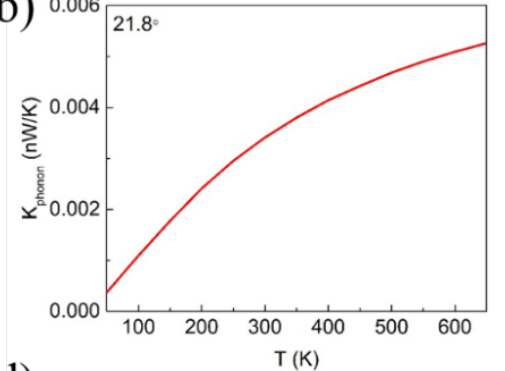

(d)

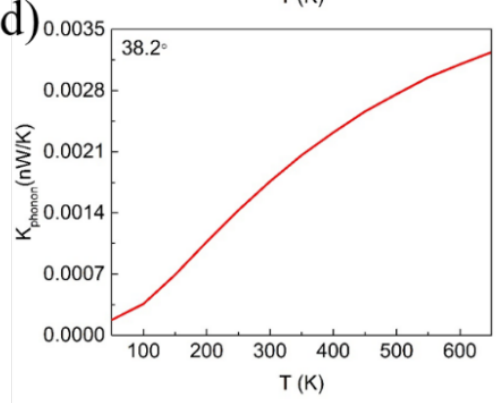

Figure 3.9. The electron and phonon contribution to the thermal conductance in $21.8^{\circ}$ (a) (b) and $38.2^{\circ}$ (c) (d) TBGNRJs.

\subsection{Conclusion}

We have investigated the electron transport and thermoelectric performance of TBGNRJs for $0^{\circ}, 21.8^{\circ}, 38.2^{\circ}$ and $60^{\circ}$ rotation angles by the first principles calculation. It is found that the $I-V$ curves of $0^{\circ}, 21.8^{\circ}$ and $38.2^{\circ}$ rotation angles exhibit behavior similar to $p-n$ junctions. However, the $60^{\circ}$ TBGNRJs is different, which exhibits an $I-V$ curve similar to a transistor. NDR is shown in TBGNRJs at $21.8^{\circ}$ and $38.2^{\circ}$ rotation angles under $\pm 0.2 \mathrm{~V}$ bias voltage. High $Z T$ values of 2.0, 2.7 and 6.1 have been achieved at $-0.25 \mathrm{eV}, 0.33 \mathrm{eV}$ and $0.68 \mathrm{eV}$ for the $21.8^{\circ}$ rotation angles at $300 \mathrm{~K}$. It is interpreted that the reason of high $Z T$ values of $21.8^{\circ}$ TBGNRJ is due to its wider bandgap, transmission gap, electrical conduction gap and a strong reduction of thermal conductance for the TBGNRJs compared with the single GNR. Moreover, the phonon contribution to thermal conductance is much more than that of electrons in $Z T$ values of 2.0 and 2.7 in the $21.8^{\circ}$ TBGNRJs. At the $Z T$ value of 6.1 , the electron contribution to thermal conductance is comparable to that of phonons. The outstanding $Z T$ 
values of TBGNRJs make it a promising device structure for thermoelectric applications.

\subsection{References}

[1] K.S. Novoselov, A.K. Geim, S.V. Morozov, D. Jiang, Y. Zhang, S.V. Dubonos, I.V. Grigorieva, A.A. Firsov, Electric field effect in atomically thin carbon films, Science 306(5696) (2004) 666669.

[2] K.I. Bolotin, K.J. Sikes, Z. Jiang, M. Klima, G. Fudenberg, J. Hone, P. Kim, H.L. Stormer, Ultrahigh electron mobility in suspended graphene, Solid State Commun. 146(9-10) (2008) 351-355.

[3] L. Banszerus, M. Schmitz, S. Engels, J. Dauber, M. Oellers, F. Haupt, K. Watanabe, T. Taniguchi, B. Beschoten, C. Stampfer, Ultrahigh-mobility graphene devices from chemical vapor deposition on reusable copper, Sci. Adv. 1(6) (2015).

[4] L. Banszerus, M. Schmitz, S. Engels, M. Goldsche, K. Watanabe, T. Taniguch, B. Beschoten, C. Stampfer, Ballistic Transport Exceeding 28 mu m in CVD Grown Graphene, Nano Lett. 16(2) (2016) 1387-1391.

[5] Y.M. Zuev, W. Chang, P. Kim, Thermoelectric and Magnetothermoelectric Transport Measurements of Graphene, Phys. Rev. Lett. 102(9) (2009).

[6] A.H. Castro Neto, F. Guinea, N.M.R. Peres, K.S. Novoselov, A.K. Geim, The electronic properties of graphene, Rev. Mod. Phys. 81(1) (2009) 109-162.

[7] A.A. Balandin, S. Ghosh, W.Z. Bao, I. Calizo, D. Teweldebrhan, F. Miao, C.N. Lau, Superior thermal conductivity of single-layer graphene, Nano Lett. 8(3) (2008) 902-907.

[8] K.S. Kim, Y. Zhao, H. Jang, S.Y. Lee, J.M. Kim, K.S. Kim, J.H. Ahn, P. Kim, J.Y. Choi, B.H. Hong, Large-scale pattern growth of graphene films for stretchable transparent electrodes, Nature 457(7230) (2009) 706-710.

[9] H. Jang, Y.J. Park, X. Chen, T. Das, M.S. Kim, J.H. Ahn, Graphene-Based Flexible and Stretchable Electronics, Adv. Mater. 28(22) (2016) 4184-4202.

[10] T.A. Amollo, G.T. Mola, M.S.K. Kirui, V.O. Nyamori, Graphene for Thermoelectric Applications: Prospects and Challenges, Crit. Rev. Solid State Mater. Sci. 43(2) (2018) 133- 
157.

[11] C.B. Vining, An inconvenient truth about thermoelectrics, Nat. Mater. 8(2) (2009) 83-85.

[12] Y. Xu, Z.Y. Li, W.H. Duan, Thermal and Thermoelectric Properties of Graphene, Small 10(11) (2014) 2182-2199.

[13] L.D. Hicks, M.S. Dresselhaus, Effect of Quantum-Well Structures on the Thermoelectric Figure of Merit, Phys. Rev. B 47(19) (1993) 12727-12731.

[14] L.D. Hicks, M.S. Dresselhaus, Thermoelectric Figure of Merit of a One-Dimensional Conductor, Phys. Rev. B 47(24) (1993) 16631-16634.

[15] H. Zheng, H.J. Liu, X.J. Tan, H.Y. Lv, L. Pan, J. Shi, X.F. Tang, Enhanced thermoelectric performance of graphene nanoribbons, Appl. Phys. Lett. 100(9) (2012).

[16] S.H. Tan, K.Q. Chen, The enhancement of the thermoelectric performance in zigzag graphene nanoribbon by edge states, Carbon 94 (2015) 942-945.

[17] L. Zhang, Z.Z. Yu, F.M. Xu, J. Wang, Influence of dephasing and B/N doping on valley Seebeck effect in zigzag graphene nanoribbons, Carbon 126 (2018) 183-189.

[18] Y.J. Ouyang, J. Guo, A theoretical study on thermoelectric properties of graphene nanoribbons, Appl. Phys. Lett. 94(26) (2009).

[19] H. Sevincli, C. Sevik, T. Cagin, G. Cuniberti, A bottom-up route to enhance thermoelectric figures of merit in graphene nanoribbons, Sci. Rep. 3 (2013).

[20] V.H. Nguyen, M.C. Nguyen, H.V. Nguyen, J. Saint-Martin, P. Dollfus, Enhanced thermoelectric figure of merit in vertical graphene junctions, Appl. Phys. Lett. 105(13) (2014). [21] X.K. Gu, Y.J. Wei, X.B. Yin, B.W. Li, R.G. Yang, Colloquium: Phononic thermal properties of two-dimensional materials, Rev. Mod. Phys. 90(4) (2018).

[22] G. Pennelli, Review of nanostructured devices for thermoelectric applications, Beilstein J Nanotech 5 (2014) 1268-1284.

[23] B.L. Li, K.Q. Chen, Effects of electron-phonon interactions on the spin-dependent Seebeck effect in graphene nanoribbons, Carbon 119 (2017) 548-554.

[24] Z.Z. Yu, F.M. Xu, J. Wang, Valley Seebeck effect in gate tunable zigzag graphene nanoribbons, Carbon 99 (2016) 451-455.

[25] Z. Zhu, Z.G. Fthenakis, D. Tomanek, Electronic structure and transport in graphene/haeckelite hybrids: an ab initio study, 2d Materials 2(3) (2015). 
[26] S.I. Vishkayi, M.B. Tagani, H.R. Soleimani, Enhancement of thermoelectric efficiency by embedding hexagonal boron-nitride cells in zigzag graphene nanoribbons, J Phys D Appl Phys 48(23) (2015).

[27] M.S. Hossain, F. Al-Dirini, F.M. Hossain, E. Skafidas, High Performance Graphene Nanoribbon Thermoelectric Devices by Incorporation and Dimensional Tuning of Nanopores, Sci. Rep. 5 (2015).

[28] V.T. Tran, J. Saint-Martin, P. Dollfus, S. Volz, Optimizing the thermoelectric performance of graphene nano-ribbons without degrading the electronic properties, Sci. Rep. 7 (2017).

[29] Y. Anno, Y. Imakita, K. Takei, S. Akita, T. Arie, Enhancement of graphene thermoelectric performance through defect engineering, 2d Materials 4(2) (2017).

[30] W.W. Zhao, Y.L. Wang, Z.T. Wu, W.H. Wang, K.D. Bi, Z. Liang, J.K. Yang, Y.F. Chen, Z.P. Xu, Z.H. Ni, Defect-Engineered Heat Transport in Graphene: A Route to High Efficient Thermal Rectification, Sci. Rep. 5 (2015).

[31] Y. Cao, V. Fatemi, A. Demir, S. Fang, S.L. Tomarken, J.Y. Luo, J.D. Sanchez-Yamagishi, K. Watanabe, T. Taniguchi, E. Kaxiras, R.C. Ashoori, P. Jarillo-Herrero, Correlated insulator behaviour at half-filling in magic-angle graphene superlattices, Nature 556(7699) (2018) 80-+. [32] S.J. Ahn, P. Moon, T.H. Kim, H.W. Kim, H.C. Shin, E.H. Kim, H.W. Cha, S.J. Kahng, P. Kim, M. Koshino, Y.W. Son, C.W. Yang, J.R. Ahn, Dirac electrons in a dodecagonal graphene quasicrystal, Science 361(6404) (2018) 782-+.

[33] W. Yao, E.Y. Wang, C.H. Bao, Y.O. Zhang, K.A. Zhang, K.J. Bao, C.K. Chan, C.Y. Chen, J. Avila, M.C. Asensio, J.Y. Zhu, S.Y. Zhou, Quasicrystalline 30 degrees twisted bilayer graphene as an incommensurate superlattice with strong interlayer coupling, Proc. Natl. Acad. Sci. U.S.A. $115(27)(2018) 6928-6933$.

[34] Y. Cao, V. Fatemi, S. Fang, K. Watanabe, T. Taniguchi, E. Kaxiras, P. Jarillo-Herrero, Unconventional superconductivity in magic-angle graphene superlattices, Nature 556(7699) (2018) 43-+.

[35] M. Pelc, E.S. Morell, L. Brey, L. Chico, Electronic Conductance of Twisted Bilayer Nanoribbon Flakes, J. Phys. Chem. C 119(18) (2015) 10076-10084.

[36] L. Jelver, P.M. Larsen, D. Stradi, K. Stokbro, K.W. Jacobsen, Determination of low-strain interfaces via geometric matching, Phys. Rev. B 96(8) (2017). 
[37] C.Y. Li, B. Debnath, X.J. Tan, S.S. Su, K. Xu, S.P. Ge, M.R. Neupane, R.K. Lake, Commensurate lattice constant dependent thermal conductivity of misoriented bilayer graphene, Carbon 138 (2018) 451-457.

[38] Atomistix ToolKit (ATK), https://quantumwise.com/. .

[39] S. Grimme, Semiempirical GGA-type density functional constructed with a long-range dispersion correction, J. Comput. Chem. 27(15) (2006) 1787-1799.

[40] A. Mohammadi, S. Haji-Nasiri, The electronic transport properties of defected bilayer sliding armchair graphene nanoribbons, Phys. Lett. A 382(15) (2018) 1040-1046.

[41] F. Gargiulo, O.V. Yazyev, Structural and electronic transformation in low-angle twisted bilayer graphene, 2d Materials 5(1) (2018).

[42] J.M.B.L. dos Santos, N.M.R. Peres, A.H. Castro, Graphene bilayer with a twist: Electronic structure, Phys. Rev. Lett. 99(25) (2007).

[43] N. Lu, H.Y. Guo, Z.W. Zhuo, L. Wang, X.J. Wu, X.C. Zeng, Twisted MX2/MoS2 heterobilayers: effect of van der Waals interaction on the electronic structure, Nanoscale 9(48) (2017) 19131-19138.

[44] M. Buttiker, Y. Imry, R. Landauer, S. Pinhas, Generalized Many-Channel Conductance Formula with Application to Small Rings, Phys. Rev. B 31(10) (1985) 6207-6215.

[45] Y.J. Li, M.D. Li, J.S. Liu, Q.Q. Sun, P. Zhou, P.F. Wang, S.J. Ding, D.W. Zhang, Atomic scale investigation of the abnormal transport properties in bilayer graphene nanoribbon, Appl. Phys. Lett. 100(1) (2012).

[46] K.M.M. Habib, S. Ahsan, R.K. Lake, Computational Study of Negative Differential Resistance in Graphene Bilayer Nanostructures, Proc Spie 8101 (2011).

[47] J. Kumar, H.B. Nemade, P.K. Giri, Density functional theory investigation of negative differential resistance and efficient spin filtering in niobium-doped armchair graphene nanoribbons, Phys. Chem. Chem. Phys. 19(43) (2017) 29685-29692.

[48] D. Zhang, M.Q. Long, X.J. Zhang, L.L. Cui, X.M. Li, H. Xu, Perfect spin filtering, rectifying and negative differential resistance effects in armchair graphene nanoribbons, J. Appl. Phys. 121(9) (2017).

[49] S. Li, C.K. Gan, Y.W. Son, Y.P. Feng, S.Y. Quek, Low-bias negative differential resistance effect in armchair graphene nanoribbon junctions, Appl. Phys. Lett. 106(1) (2015). 
[50] H. Sadeghi, S. Sangtarash, C.J. Lambert, Oligoyne Molecular Junctions for Efficient Room Temperature Thermoelectric Power Generation, Nano Lett. 15(11) (2015) 7467-7472.

[51] J.G. Checkelsky, N.P. Ong, Thermopower and Nernst effect in graphene in a magnetic field, Phys. Rev. B 80(8) (2009).

[52] H.L. Liu, X. Shi, F.F. Xu, L.L. Zhang, W.Q. Zhang, L.D. Chen, Q. Li, C. Uher, T. Day, G.J. Snyder, Copper ion liquid-like thermoelectrics, Nat. Mater. 11(5) (2012) 422-425.

[53] L. Han, D.V. Christensen, A. Bhowmik, S.B. Simonsen, L.T. Hung, E. Abdellahi, Y.Z. Chen, N.V. Nong, S. Linderoth, N. Pryds, Scandium-doped zinc cadmium oxide as a new stable ntype oxide thermoelectric material, J Mater Chem A 4(31) (2016) 12221-12231.

[54] R. Chetty, A. Bali, R.C. Mallik, Tetrahedrites as thermoelectric materials: an overview, J. Mater. Chem. C 3(48) (2015) 12364-12378.

[55] Y. Yokomizo, J. Nakamura, Giant Seebeck coefficient of the graphene/h-BN superlattices, Appl. Phys. Lett. 103(11) (2013).

[56] J.M. Zheng, P. Guo, Z.Y. Ren, Z.Y. Jiang, J.T. Bai, Z.Y. Zhang, Conductance fluctuations as a function of sliding motion in bilayer graphene nanoribbon junction: A first-principles investigation, Appl. Phys. Lett. 101(8) (2012).

[57] G.J. Snyder, E.S. Toberer, Complex thermoelectric materials, Nat. Mater. 7(2) (2008) 105114.

[58] K. Nakada, M. Fujita, G. Dresselhaus, M.S. Dresselhaus, Edge state in graphene ribbons: Nanometer size effect and edge shape dependence, Phys. Rev. B 54(24) (1996) 17954-17961. [59] X.T. Jia, M. Hofmann, V. Meunier, B.G. Sumpter, J. Campos-Delgado, J.M. Romo-Herrera, H.B. Son, Y.P. Hsieh, A. Reina, J. Kong, M. Terrones, M.S. Dresselhaus, Controlled Formation of Sharp Zigzag and Armchair Edges in Graphitic Nanoribbons, Science 323(5922) (2009) 1701-1705.

[60] J. Lahiri, Y. Lin, P. Bozkurt, I.I. Oleynik, M. Batzill, An extended defect in graphene as a metallic wire, Nat. Nanotechnol. 5(5) (2010) 326-329.

[61] H. Sadeghi, S. Sangtarash, C.J. Lambert, Enhanced Thermoelectric Efficiency of Porous Silicene Nanoribbons, Sci. Rep. 5 (2015).

[62] H. Sadeghi, S. Sangtarash, C.J. Lambert, Enhancing the thermoelectric figure of merit in engineered graphene nanoribbons, Beilstein J Nanotech 6 (2015) 1176-1182. 


\section{Chapter4: Study of electronic and optical properties of twisted bilayer GaN structures by using first principles theory}

Gallium Nitride ( $\mathrm{GaN}$ ) is one of well investigated materials contributing to many advanced power electronic and optoelectronic devices due to its wide bandgap. However, the derivatives of its monolayer form such as bilayer structures have rarely been reported. Here we report electronic and optical properties of the GaN bilayer structures that are rotated in plane at several optimized rotation angles by using the density functional theory method. For the aim of maintaining the structural stability and using a small cell size, the twisting angles of the GaN bilayer structures are optimized to be $27.8^{\circ}, 38.2^{\circ}$ and $46.8^{\circ}$ using the crystal matching theory. From the bandstructure analysis, the bandgap is increased for twisted structures compared with the non-twisting case. Simulation results show that values of absorption coefficient, extinction coefficient, reflectivity and the refractive index at these angles. The spectra of all above optical properties match with the bandgap values. The refractive index of the bilayer structures at all twisting angles including $0^{\circ}$ has been simulated to be smaller than the bulk GaN, indicating a reduced scattering loss in optoelectronics applications. Considering the analysed results, the possible applications can be low loss integrated electronic and optical devices and systems.

\subsection{Introduction}


GaN is one of typical III-V compound semiconductor materials. It has been widely applied in power semiconductor and optoelectronic devices. For optical applications, GaN can be used as the substrate to make violet (405 nm) laser diode. Light-emitting diodes (LEDs) made with GaN could achieve high brightness and cover the range of primary colors and it is applied to daylight visible full-color LED displays and white LEDs. Because of the low sensitivity to ionizing radiation, GaN based solar cell arrays are suitable for satellites. The high working temperature and voltage limit of GaN transistors are higher than those of gallium arsenide (GaAs) transistors. This leads to many applications in microwave power amplifier devices [1-4]. GaN has becoming one of important materials in the next generation smart phones and internet technologies as it can meet the requirements of high power, high temperature, low refractive index (2.3 for GaN compared with 3.5 for GaAs), and superior temperature stability of the refractive index (one order of magnitude higher than that of $\ln P)$.

It was reported in the past research that the suspended bilayer structural devices have advantages of enhanced electronic performances. The twodimensional (2D) form of materials are deemed to have much thinner thickness than the bulk form, which brings different electronic, mechanical, and optical performances compared with the bulk form [5-15]. This is due to the change of crystallization and quantum effect, which is the special behavior and principles under nano scale and these are quite different from those under classical bulk scale, in the 2D materials. Some previous work has been conducted on the density functional theory of 2D materials to investigate their electronic properties [16]. It was reported in [17] that a single layer III-V binary compound semiconductor - GaN has been simulated using the first principles method. The band structures of the single layer GaN could be controlled by chemical functionalization [18, 19], adsorption of non-metal materials [20-22] [23] , vacancy $[24,25]$ and doping $[26,27]$ methods. Those results indicated that $2 \mathrm{D}$ 
GaN is indeed a future development trend for high performance nanoelectronic devices. Experimentally it was reported in $[28,29]$ that the 2D GaN can be synthesized by the migration-enhanced encapsulated growth (MEEG) technique utilizing epitaxial graphene. It can also be grown by using the surface-confined nitridation reaction method on liquid metals [30], which demonstrated the potential to implement 2D GaN in real nanoelectronic devices [23] . By stacking two single layer materials with the van der Waals force, and rotating one of them against the other layer, the whole structure may display interesting characteristics. This was firstly reported in [31, 32], which described that a special rotating/twisting angle of bilayer graphene exhibits superior electrical conductivity. In their work, the twisting angle could be controlled by using the graphene produced by the exfoliation method. These previous papers on the twisting effect of stacked 2D materials stimulated researchers to conduct studies on other multi-layer structures based on various monolayer materials, including heterostructures. Specifically, for the 2D GaN, the investigation has been performed using the first principles method in the work on the twisting effect of bilayer GaN. The twisted GaN bilayer model is built and subsequently analyzed to unveil its electronic and optical properties. These results will lead to wider and deeper explorations of single layer GaN in the hope of applying them in the practical electronic and optoelectronic devices.

\subsection{Computational method}

The modelling of the GaN bilayer structure is based on the Quantum Atomistix ToolKit simulation tools. The simulations are conducted using density functional theory (DFT). The method of generalized gradient approximation (GGA) with the Perdew-Burke-Ernzerhof (PBE) parameterization at temperature of 300K, mesh cut-off energy of 50 Hartree and $11 \times 11 \times 1$ k-points grid were used for optimizing all the structures. Considering with the influence of vdW force 
applied to the bilayers, the semi-empirical corrections by Grimme DFT-D2 model was used. This would involve the long-range van der Waals interaction. Then we added a large vacuum spacing (> $20 \AA$ ) along the c-direction in the structure to avoid periodic boundary conditions from being applied in the cdirection. The maximum force on each atom is set to structure $0.01 \mathrm{eV} / \AA$ for structural relaxation calculations. In the electronic and optical properties simulation part, we use TB09 meta-GGA method for bandgap, projected density of state and optical properties calculations with $c$ parameter of 1.76 . The $c$ parameter is a constant value used in TB09 mete-GGA method. Figure 4.1 is the calculated $c$ parameters against the calculated bandgap. The $c$ value is calculated to match the experimental bandgap of $4.98 \mathrm{eV}$ reported in [28] for the two-monolayer structures (non-twisting). There was so far no report of the bandgap for vdW bonded two GaN monolayers. The vdW bonding has less effect on the bandgap of the monolayer structure. It was reported that the vdW interaction does not affect the properties close to Fermi level [33] for the vdW bonded heterostructures, indicating the bandgap of the vdW bonded GaN bilayer should be similar as that of the monolayer. The side views of the GaN bilayer structure are shown in Figure 4.2. From the side view part, the structure is built by stacking two layers of 2D GaN cell. Figure 4.2 also shows the bottom view of different rotated structures. Rotation angles were selected according to the criteria of optimized stress and atom number, with the aim of achieving the most stable structures. The method of selecting optimal rotation angles was described in [34]. Generally speaking, for the twisted bilayer structure, the number of atoms in single cell increases while the rotation angle increases. Table 1 gives the detailed number of atoms and bandgap in each structure. However, when the angle equals to $38.21^{\circ}$, the atom number decreases to 56 , which is much less than those with rotation angles of $27.8^{\circ}$ and $46.8^{\circ}$. All models are passivated with hydrogen atoms. Figure 4.3 shows the band structures of the atomic model and the projected density of states (PDOS) for four rotation angles of the GaN bilayer structures. The bilayer structures have 
bandgaps of $4.97 \mathrm{eV}, 5.06 \mathrm{eV}, 5.25 \mathrm{eV}$, and $5.22 \mathrm{eV}$ under the angles of $0^{\circ}$, $27.8^{\circ}, 38.2^{\circ}$, and $46.8^{\circ}$ respectively. From the bandgap, the bilayer GaN displays typical wide bandgap semiconducting property. The bandgap value is significantly larger than the value of the bulk $\mathrm{GaN}$, of which is typical $3.4 \mathrm{eV}$. For all the structures, both the valence band maximum (VBM) and conduction band minimum (CBM) occur at Gamma point, which indicates the bandgap is the direct type. According to the PDOS results, the VBM is primarily composed of $\mathrm{N} 2 \mathrm{p}, \mathrm{H}$ 1s orbitals and the CBM is a hybridization of $\mathrm{Ga} 3 \mathrm{~s}, \mathrm{Ga} 3 \mathrm{p}, \mathrm{N} 2 \mathrm{~s}$, and $\mathrm{H}$ 1s orbitals, however Ga $3 p$ contribute the most. The CBM occurs in orbitals $\mathrm{Ga} 3 p$ and the VBM occurs in N 2p. Figure 4.4 displays the results of the bandgap, CBM and VBM against rotation angles. While the rotation angle increases, the bandgap increases (from $0^{\circ}$ to $38.2^{\circ}$ ) then slightly decrease (from $38.2^{\circ}$ to $46.8^{\circ}$ ). The CBM and VBM remain at point G upon the increasing of rotation angle. However, when the angles are $27.8^{\circ}$ or $38.2^{\circ}$, the absolute value of CBM is larger than that of VBM, which indicates that the Fermi level moves towards to the valence band. 


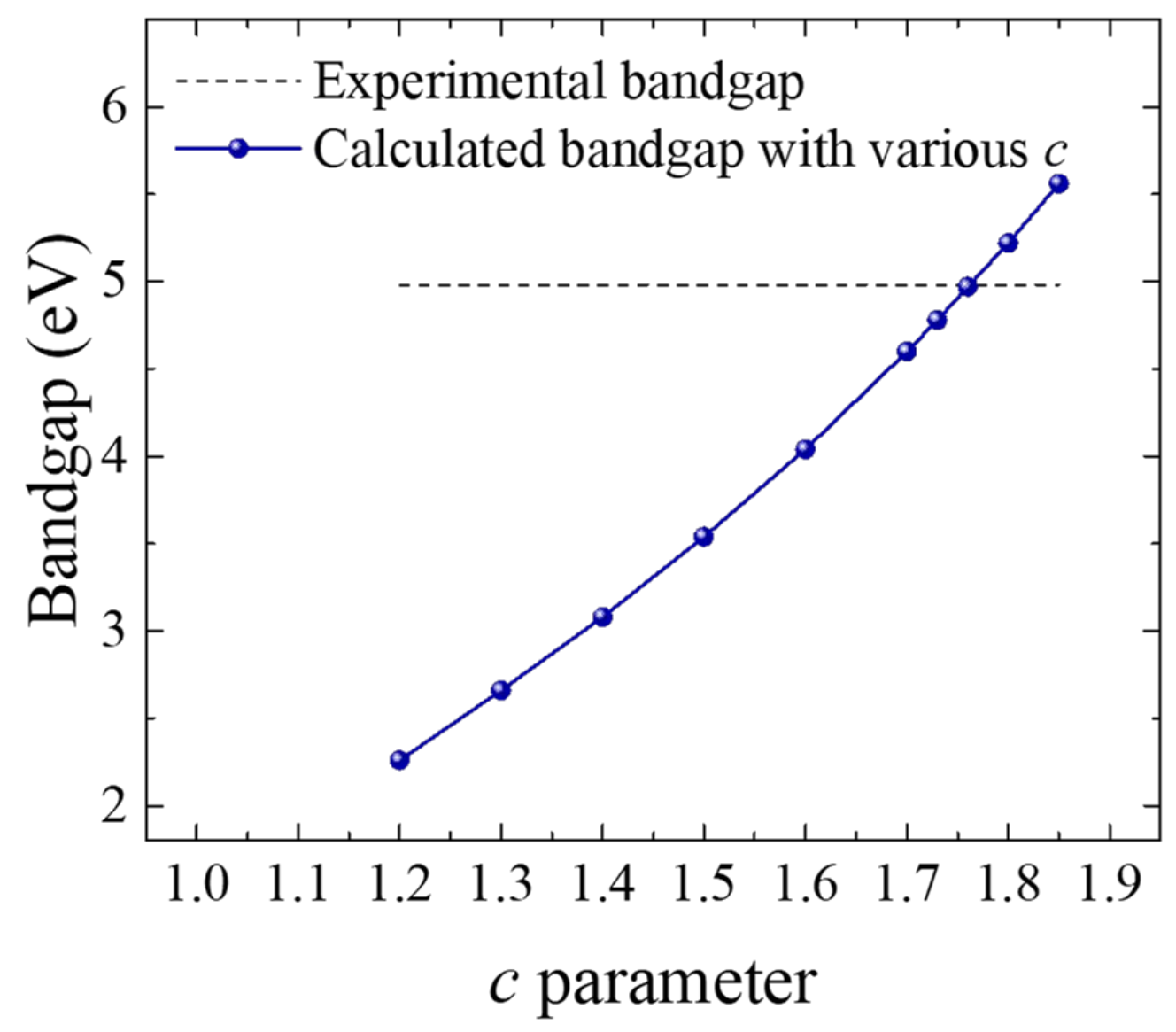

Figure 4.1. bandgap of single layer $2 D$ GaN vs. c parameter
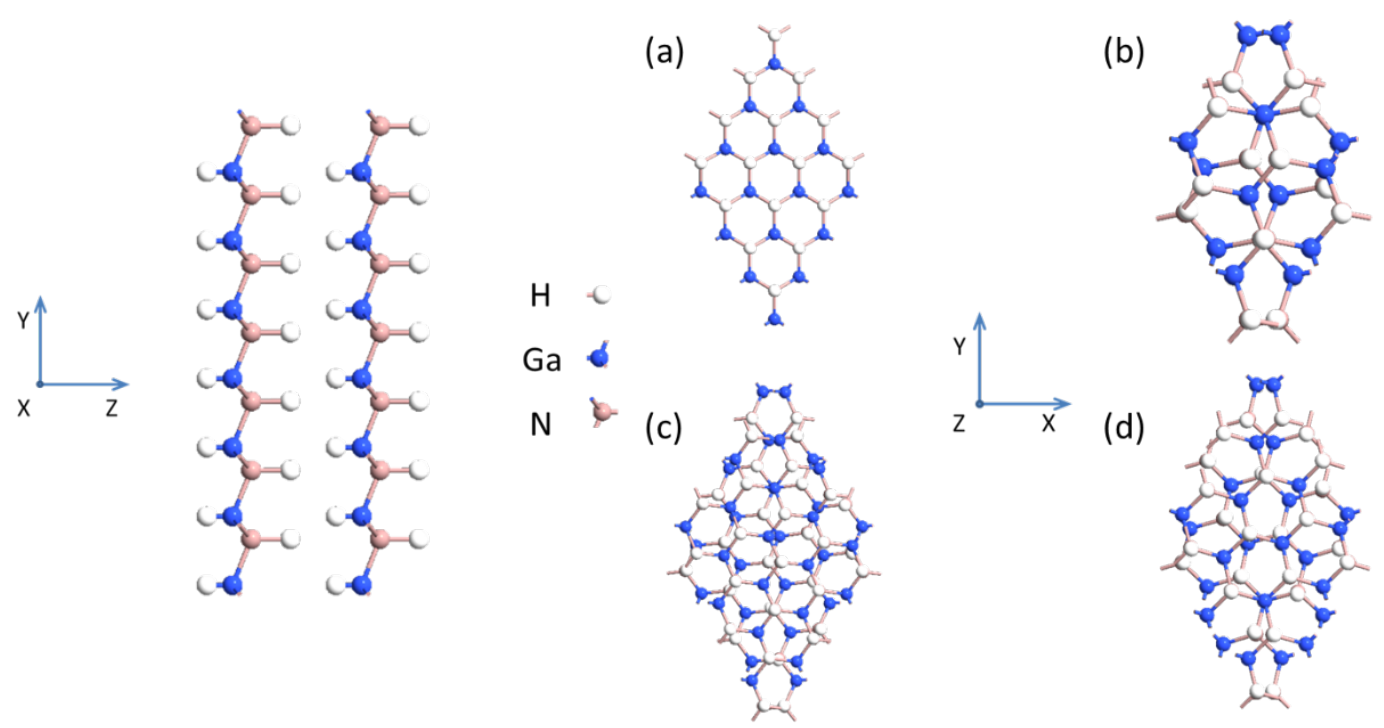

Figure 4.2. The side view of the rotation structure and (a)The bottom view of $0^{\circ}$ rotation structure (b) The bottom view of $38.2^{\circ}$ rotation structure (c) The bottom view of $27.8^{\circ}$ rotation structure (d) The bottom view of $46.8^{\circ}$ rotation structure 
Table1. Atom numbers and bandgap value of different rotation structure

\begin{tabular}{lllll}
\hline $\begin{array}{l}\text { Rotation between } \\
\text { surface(degree) }\end{array}$ & $\mathbf{0}$ & $\mathbf{2 7 . 8}$ & $\mathbf{3 8 . 2 1}$ & $\mathbf{4 6 . 8 3}$ \\
\hline $\begin{array}{l}\text { Atom numbers in } \\
\text { single cell }\end{array}$ & 8 & 104 & 56 & 152 \\
$\begin{array}{l}\text { Angle between } \\
\text { vectors(degree) }\end{array}$ & 60 & 60 & 60 & 60 \\
$\begin{array}{l}\text { Bandgap(eV) } \\
\text { (e) }\end{array}$ & 4.97 & 5.06 & 5.25 & 5.22 \\
\hline
\end{tabular}
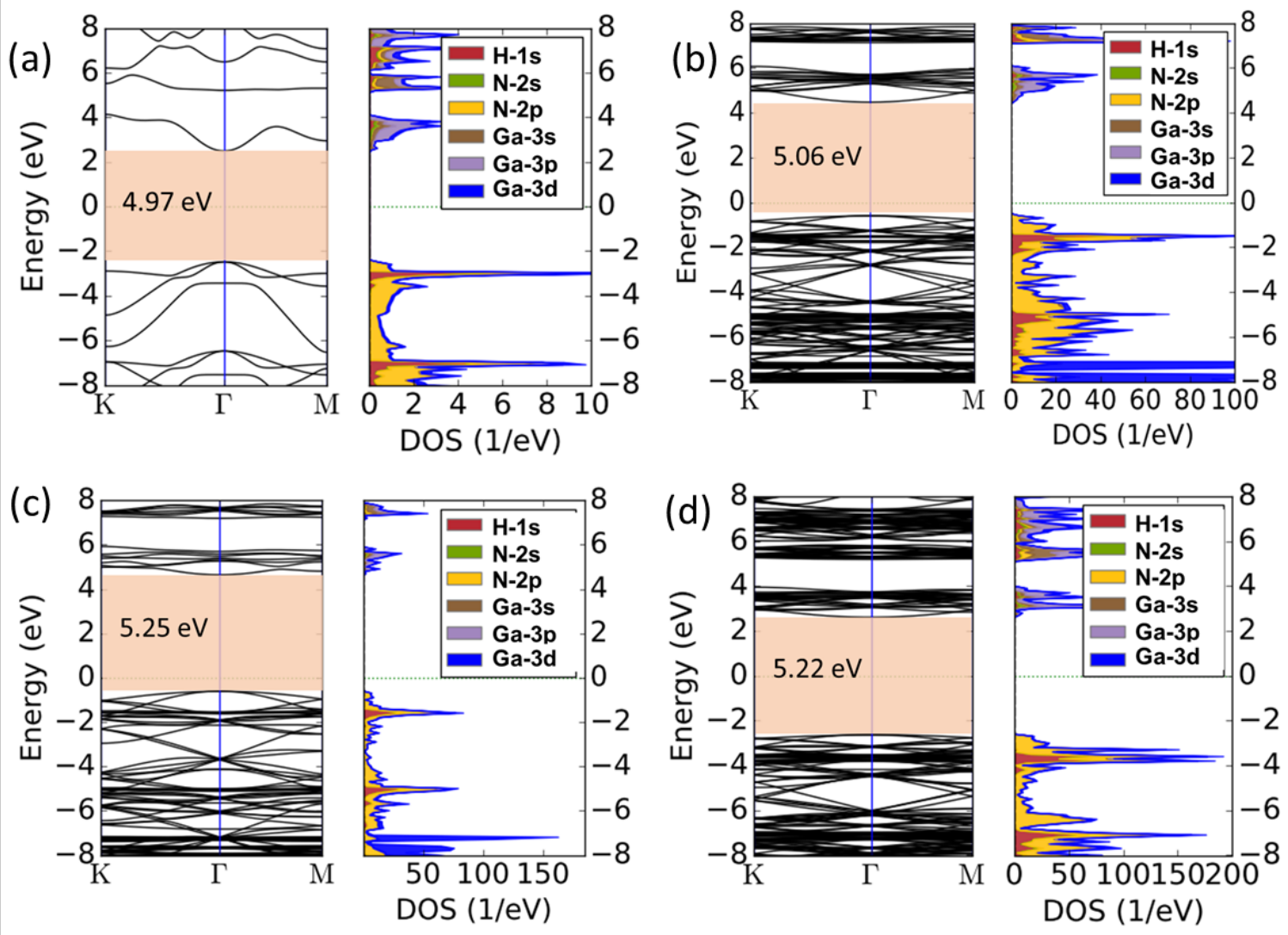

Figure 4.3. (a) the bandgap and PDOS of $0^{\circ}$ rotation structure (b) the bandgap and PDOS of $27.8^{\circ}$ rotation structure (c) the bandgap and PDOS of $38.2^{\circ}$ rotation structure (d)the bandgap and PDOS of $46.8^{\circ}$ rotation structure 


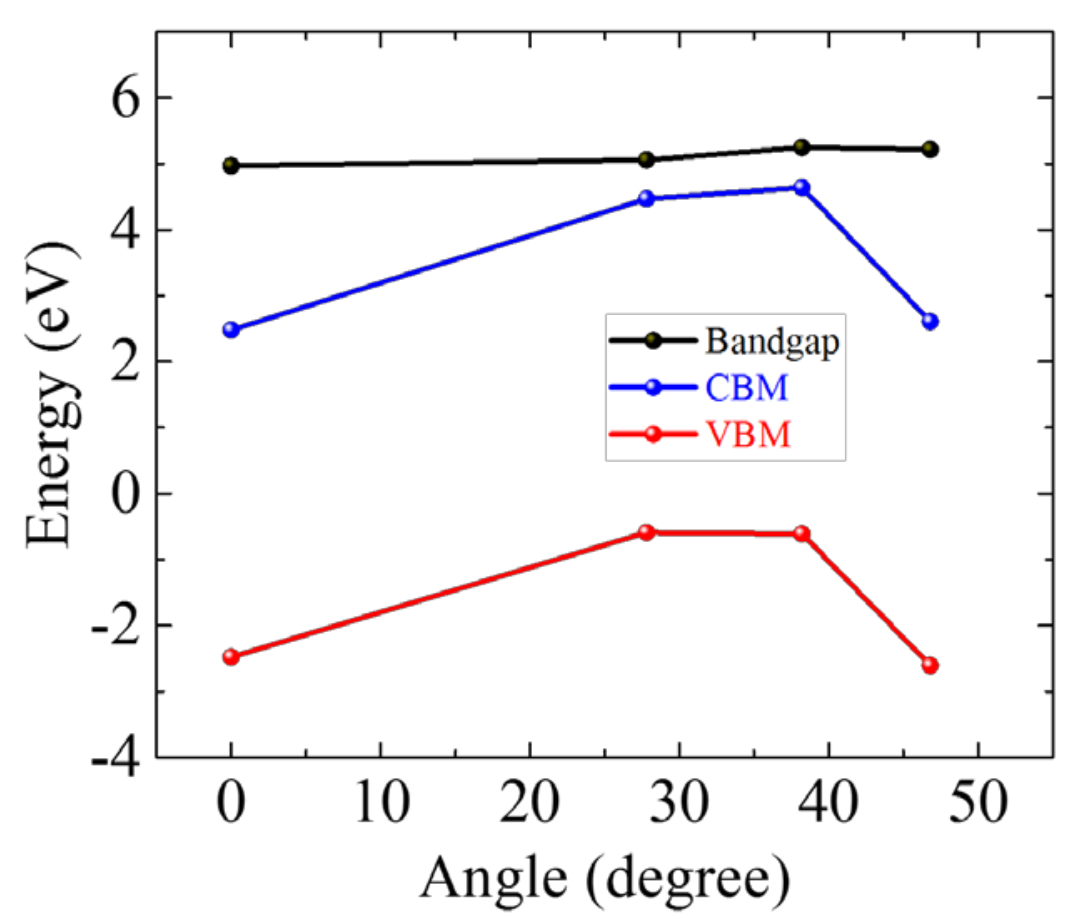

Figure 4.4. the bandgap, $C B M$ and $C B M$ vs rotation angle

\subsection{Optional properties}

The bilayer GaN models are calculated to find out several key optical properties such as absorption coefficient and refractive index using the general mathematic procedure. [35] . The susceptibility tensor at a frequency $\omega$, which characterizes the polarization for a material under an electrical field, can be written using the Kubo-Greenwood formula:

$$
\chi_{i j}(\omega)=-\frac{e^{2} \hbar^{4}}{m^{2} \varepsilon_{0} V \omega^{2}} \sum_{n m} \frac{f\left(E_{m}\right)-f\left(E_{n}\right)}{E_{n m}-\hbar \omega-i \Gamma} \pi_{n m}^{i} \pi_{m n}^{j}(4.1)
$$

where $E_{n}, E_{m}$ and $E_{n m}$ represent the energy levels at energy states $n, m$ and energy difference between $n$ and $m . f \Gamma$ and $V$ stand for the Fermi function, broadening and volume, respectively. $\pi_{n m}^{i}$ is the $i$-th component of dipole matrix element between states $n$ and $m . \varepsilon_{0}, \hbar$ and $e$ represent the permittivity in vacuum, reduced Planck's constant and single electron charge, respectively. The susceptibility tensor is used to derive the relative permittivity $\left(\varepsilon_{r}\right)$, which also determines the amount of polarization of a dielectric under external electric 
field. It is

$$
\varepsilon_{r}(\omega)=(1+\chi(\omega))(4.2)
$$

The polarization response of a material like GaN under an electric field should be expressed in complex form as there always be a phase change to the frequency of the electric field. Simple expression for a complex permittivity is for the frequency $\omega$ is

$$
\varepsilon_{r}(\omega)=\varepsilon_{0} \varepsilon_{r}(\omega)=\varepsilon^{\prime}(\omega)+\varepsilon^{\prime \prime}(\omega)(4.3)
$$

where $\varepsilon_{0}, \varepsilon^{\prime}$ and $\varepsilon^{\prime \prime}$ are the vacuum permittivity, real and imaginary parts of the complex permittivity, respectively. Further expressions for the complex terms of the permittivity can be obtained from the Kramers-Kronig relations (bidirectional mathematical relations, connecting the real and imaginary parts of any complex function), and shown as

$$
\begin{gathered}
\varepsilon^{\prime}(\omega)=1+\frac{2}{\pi} A \int_{0}^{\infty} \frac{\omega^{\prime} \varepsilon^{\prime \prime}\left(\omega^{\prime}\right)}{\omega^{\prime 2}-\omega^{2}} d \omega^{\prime} \\
\varepsilon^{\prime \prime}(\omega)=-\frac{2 \omega}{\pi} A \int_{0}^{\infty} \frac{\varepsilon^{\prime}\left(\omega^{\prime}\right)}{\omega^{\prime 2}-\omega^{2}} d \omega^{\prime}+\frac{\sigma_{0}}{\varepsilon_{0} \omega}
\end{gathered}
$$

where the $\sigma_{0}$ and $A$ denote the DC conductivity and the Cauchy principal value. Since refractive index $(n)$ and the extinction coefficient $(\kappa)$ are related to the $\varepsilon(\omega)$ according to the relation $\varepsilon(\omega)=n+i \kappa$, Hence from equations (4.4) and (4.5), the refractive index $(n)$ and the extinction coefficient $(\kappa)$ can be written as

$$
\begin{gathered}
n=\sqrt{\frac{\sqrt{\varepsilon^{\prime 2}+\varepsilon^{\prime \prime}{ }^{2}}+\varepsilon \prime}{2}}(4.6) \\
\kappa=\sqrt{\frac{\sqrt{\sqrt{\varepsilon^{2}+\varepsilon^{\prime \prime}}}-\varepsilon^{\prime \prime}}{2}}(4.7)
\end{gathered}
$$

The optical absorption coefficient $\left(\alpha_{a}\right)$ can then be written as:

$$
\alpha_{a}=2 \frac{\omega}{c} \kappa
$$

where $c$ is the speed of light and $\omega$ is the angular frequency of light. The reflectivity $(r)$ is expressed as

$$
r=\frac{(1-n)^{2}+\kappa^{2}}{(1+n)^{2}+\kappa^{2}}
$$

Figure 4.5 depicts the calculated real and imaginary parts of the complex 
permittivity of rotated GaN bilayer structures. The twisting angles at $0^{\circ}, 27.8^{\circ}$, $38.2^{\circ}$ and $46.8^{\circ}$ were calculated. In terms of the amplitude, the values at all cases are similar except that the permittivity has the maximum peak values when the rotation angle is at $38.2^{\circ}$. The peak wavelengths at all cases coincide with bandgap values, i.e. there is slight blue-shift for non-zero twisting angles. In Figure $4.5 \mathrm{a}$ the real part of the permittivity at a long wavelength $(400 \mathrm{~nm})$ for $0^{\circ}, 27.8^{\circ}, 38.2^{\circ}$ and $46.8^{\circ}$ rotation angles are $1.7322,1.7323,1.7322$ and 1.7322, respectively. The peak values of the imaginary permittivity appear between wavelength of $200 \mathrm{~nm}$ and $250 \mathrm{~nm}$ for all cases according to Figure 4.5b. Figure 4.6 shows the reflectivity, refractive index, absorption coefficient and extinction coefficient spectrums with $0^{\circ}, 27.8^{\circ}, 38.2^{\circ}$ and $46.8^{\circ}$ rotation angles respectively. Figure $4.6 \mathrm{a}$ plots the reflectivity curves against the wavelength. All cases are very similar on the reflectivity. The peak amplitude values are in the range of $10^{-8}-10^{-7}$. In Figure $4.6 \mathrm{~b}$ the reftractive index values for all cases are much smaller than the the values of bulk GaN material. In Figure $4.6 \mathrm{c}$ the maximum values of absorption coefficient in the spectrum for all cases are around $400 \mathrm{~cm}^{-1}$. In Figure $4.6 \mathrm{~d}$, the extinction coefficients of all cases follow the same trends as the absorption coefficient curves in terms of both the amplitude and wavelength. For the optical properties of the structure, it is seen a slight blue-shift as a result of twisting. This is due to that the optical bandgap increases slightly while the rotation angle increases. In order to explain these simulation results in terms of solid state physics, the similar theoretical framework as described in another paper [35] is utilized. Briefly it is understood that the Lorentz model could interpret the relations among all the above optical properties [36] . It is assumed that the bilayer structure and incident optical light are sets of oscillation systems with their harmonic resonant frequencies of $\omega_{0}$ and $\omega$, respectively. High absorption band appears when $\omega \approx \omega_{0}$. In the mismatched regions, this pseudo mechanical theory applies in this electron-photon interactive system. 
In optical applications, the attenuation of the optical signal in a material is caused by absorption, scattering, and radiation of the optical power. In particular the scattering loss is due to the refractive index caused by microscopic heterogeneities such as fluctuations in material density or composition. The optical power loss due to scattering $I_{s}$ is proportional to the refractive index $\left(I_{s} \sim n^{8}\right)$ [37], therefore a mall reduction of the refractive index can significantly reduce the scattering loss. Interestingly, the refractive index of the bilayer GaN has shown a much smaller value $(\sim 1.73)$ compared with the bulk GaN (2.3), which introduces much reduced scattering optical power loss, leading to promising application in optoelectronic devices. The optical power loss can be further reduced by the very low absorption of the bilayer structures simulated in this work, which is approximately three orders of magnitude lower compared with that of the bulk GaN materials (The absorption coefficient of the bulk GaN was reported as $\sim 10^{5} \mathrm{~cm}^{-1}$ [38]). Moreover, the bandgap of the twolayer GaN is much wider than that of bulk materials, introducing prospective applications in power electronics. Through this theoretical investigation using the first principles method, it is found that the bandgap of the bilayer GaN can be increased even wider by the twisting effect. At present, vdW force bonded bilayer materials and twisting can be achieved using the experimental method [39]. Although the experiments on bilayer twisting GaN were not found, experiments on other semiconductor materials predict that it should be feasible for the GaN.
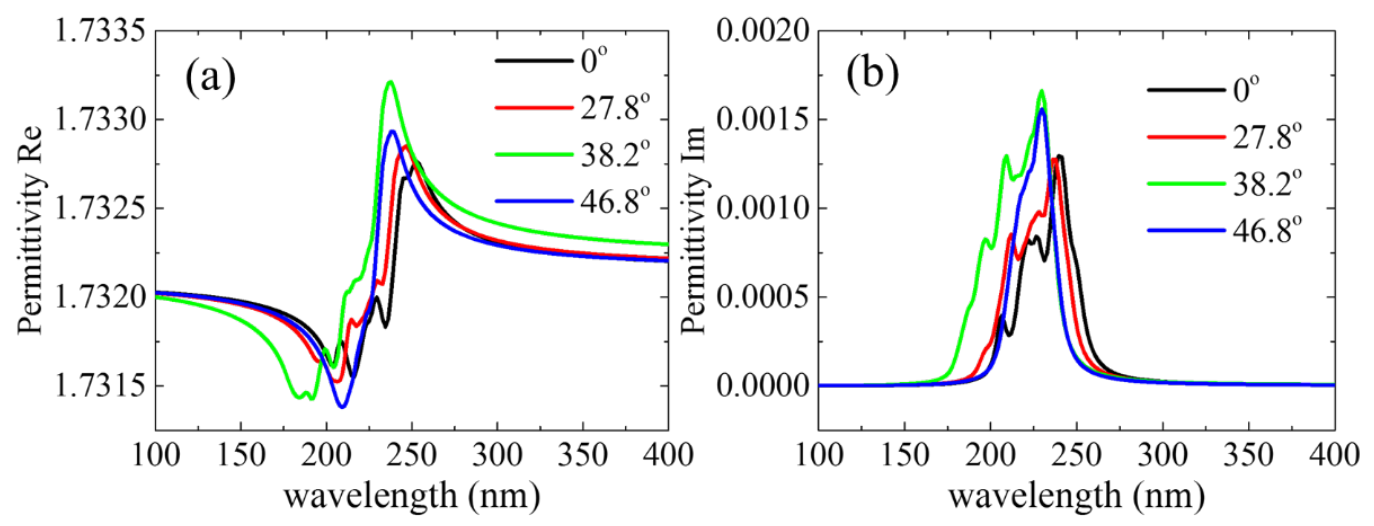
Figure 4.5 (a) the real part of permittivity (b) the imaginary part of permittivity
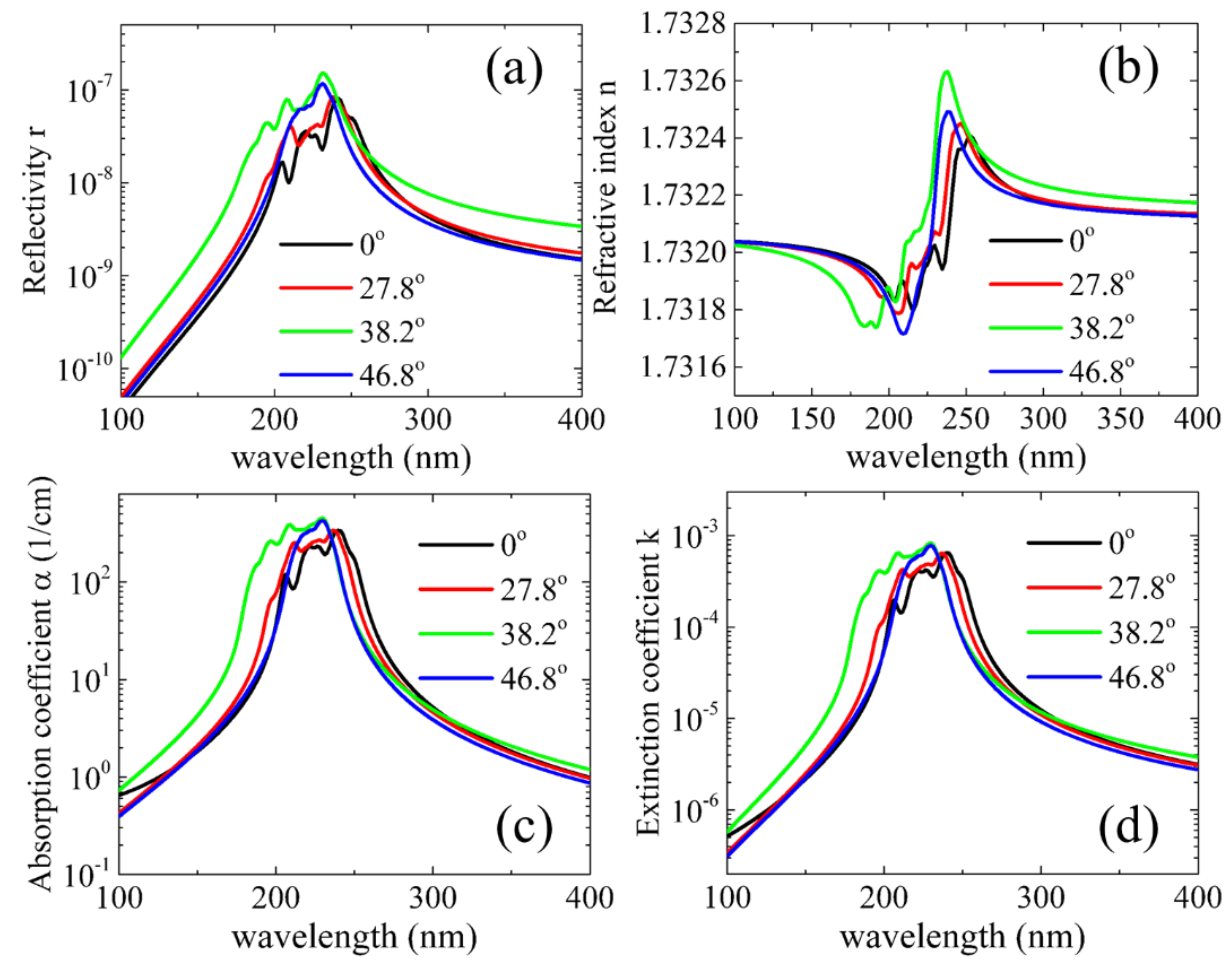

Figure 4.6 (a) the reflectivity of rotated bilayer GaN; (b) the refractive index of rotated bilayer

GaN; (c) the absorption coefficient spectrums of rotated bilayer GaN; (d) the extinction coefficient of rotated bilayer GaN.

\subsection{Conclusion}

Electronic and optical properties of the GaN bilayer structure with rotation angles $0^{\circ}, 27.8^{\circ}, 38.2^{\circ}$ and $46.8^{\circ}$ were simulated with the density functional theory. According to the first principle simulation, values of bandgap, absorption coefficient, extinction coefficient, reflectivity and the refractive index vary with the rotation angles. The two vdW bonded GaN monolayers have been found to exhibit wide bandgap, which is further increased by twisting these two layers at certain optimized angles. It is also discovered that the refractive index of the bilayer structure is much smaller compared with its bulk form. This will lead to much reduced Rayleigh scattering on the optical power. The absorption 
coefficient has significantly reduced at all twisting angles including $0^{\circ}$. The calculated optical absorption coefficient $\left(\sim 10^{2} \mathrm{~cm}^{-1}\right)$ of the bilayer $\mathrm{GaN}$ is much lower than that of the bulk GaN $\left(\sim 10^{5} \mathrm{~cm}^{-1}\right)$, reducing the power loss due to the absorption. Bilayer structure brings the advantage of increased optical bandgap and reduced optical power loss due to significant reduction of both the absorption coefficient and refractive index, which makes these bilayer GaN structures more suitable for monolithic integration of power electronic devices and optical devices. The results will be useful for future integrated low-loss optoelectronics applications.

\subsection{References}

[1] A. Avramescu, T. Lermer, J. Müller, S. Tautz, D. Queren, S. Lutgen, U. Strauß, InGaN laser diodes with $50 \mathrm{~mW}$ output power emitting at $515 \mathrm{~nm}$, Applied Physics Letters, 95 (2009) 071103. [2] M.A. Khan, J.N. Kuznia, D.T. Olson, J.M.V. Hove, M. Blasingame, L.F. Reitz, Highresponsivity photoconductive ultraviolet sensors based on insulating single-crystal GaN epilayers, Applied Physics Letters, 60 (1992) 2917-2919.

[3] C.J. Neufeld, N.G. Toledo, S.C. Cruz, M. Iza, S.P. DenBaars, U.K. Mishra, High quantum efficiency InGaN/GaN solar cells with 2.95 eV band gap, Applied Physics Letters, 93 (2008) 143502.

[4] X.H. Wang, L.W. Guo, H.Q. Jia, Z.G. Xing, Y. Wang, X.J. Pei, J.M. Zhou, H. Chen, Control performance of a single-chip white light emitting diode by adjusting strain in InGaN underlying layer, Applied Physics Letters, 94 (2009) 111913.

[5] Z.-Q. Fan, X.-W. Jiang, J. Chen, J.-W. Luo, Improving Performances of In-Plane TransitionMetal Dichalcogenide Schottky Barrier Field-Effect Transistors, ACS Applied Materials \& Interfaces, 10 (2018) 19271-19277. 
[6] Z.-Q. Fan, X.-W. Jiang, J.-W. Luo, L.-Y. Jiao, R. Huang, S.-S. Li, L.-W. Wang, In-plane Schottky-barrier field-effect transistors based on $1 \mathrm{~T} / 2 \mathrm{H}$ heterojunctions of transition-metal dichalcogenides, Physical Review B, 96 (2017) 165402.

[7] Y.-Y. Liu, B.-L. Li, S.-Z. Chen, X. Jiang, K.-Q. Chen, Effect of room temperature lattice vibration on the electron transport in graphene nanoribbons, Applied Physics Letters, 111 (2017) 133107.

[8] Y.-Y. Liu, Y.-J. Zeng, P.-Z. Jia, X.-H. Cao, X. Jiang, K.-Q. Chen, An efficient mechanism for enhancing the thermoelectricity of nanoribbons by blocking phonon transport in 2D materials, Journal of Physics: Condensed Matter, 30 (2018) 275701.

[9] K.S. Novoselov, A.K. Geim, S.V. Morozov, D. Jiang, M.I. Katsnelson, I.V. Grigorieva, S.V. Dubonos, A.A. Firsov, Two-dimensional gas of massless Dirac fermions in graphene, Nature, 438 (2005) 197-200.

[10] K.S. Novoselov, A.K. Geim, S.V. Morozov, D. Jiang, Y. Zhang, S.V. Dubonos, I.V. Grigorieva, A.A. Firsov, Electric Field Effect in Atomically Thin Carbon Films, Science, 306 (2004) 666-669. [11] B. Radisavljevic, A. Radenovic, J. Brivio, V. Giacometti, A. Kis, Single-layer MoS2 transistors, Nature Nanotechnology, 6 (2011) 147-150.

[12] C.R. Ryder, J.D. Wood, S.A. Wells, Y. Yang, D. Jariwala, T.J. Marks, G.C. Schatz, M.C. Hersam, Covalent functionalization and passivation of exfoliated black phosphorus via aryl diazonium chemistry, Nature Chemistry, 8 (2016) 597-602.

[13] L. Song, L. Ci, H. Lu, P.B. Sorokin, C. Jin, J. Ni, A.G. Kvashnin, D.G. Kvashnin, J. Lou, B.I. Yakobson, P.M. Ajayan, Large Scale Growth and Characterization of Atomic Hexagonal Boron Nitride Layers, Nano Letters, 10 (2010) 3209-3215.

[14] Y. Wang, W.-X. Zhou, L. Huang, C. Xia, L.-M. Tang, H.-X. Deng, Y. Li, K.-Q. Chen, J. Li, Z. Wei, Light induced double 'on' state anti-ambipolar behavior and self-driven photoswitching in p-WSe2/n-SnS2 heterostructures, 2D Materials, 4 (2017) 025097.

[15] S. Wu, J.S. Ross, G.-B. Liu, G. Aivazian, A. Jones, Z. Fei, W. Zhu, D. Xiao, W. Yao, D. Cobden, X. Xu, Electrical tuning of valley magnetic moment through symmetry control in bilayer MoS2, Nature Physics, 9 (2013) 149-153.

[16] S. Deng, L. Li, M. Li, Stability of direct band gap under mechanical strains for monolayer MoS2, MoSe2, WS2 and WSe2, Physica E: Low-dimensional Systems and Nanostructures, 
101 (2018) 44-49.

[17] H. Şahin, S. Cahangirov, M. Topsakal, E. Bekaroglu, E. Akturk, R.T. Senger, S. Ciraci, Monolayer honeycomb structures of group-IV elements and III-V binary compounds: Firstprinciples calculations, Physical Review B, 80 (2009) 155453.

[18] Q. Chen, H. Hu, X. Chen, J. Wang, Tailoring band gap in GaN sheet by chemical modification and electric field: Ab initio calculations, Applied Physics Letters, 98 (2011) 053102. [19] W.-Z. Xiao, L.-L. Wang, L. Xu, Q. Wan, A.-L. Pan, H.-Q. Deng, Ferromagnetic and metallic properties of the semihydrogenated GaN sheet, physica status solidi (b), 248 (2011) 1442-1445. [20] Y. Mu, Chemical Functionalization of GaN Monolayer by Adatom Adsorption, The Journal of Physical Chemistry C, 119 (2015) 20911-20916.

[21] W. Tang, M. Sun, J. Yu, J.-P. Chou, Magnetism in non-metal atoms adsorbed graphenelike gallium nitride monolayers, Applied Surface Science, 427 (2018) 609-612.

[22] X. Zhang, L. Jin, X. Dai, G. Chen, G. Liu, Two-Dimensional GaN: An Excellent Electrode Material Providing Fast Ion Diffusion and High Storage Capacity for Li-Ion and Na-Ion Batteries, ACS Applied Materials \& Interfaces, 10 (2018) 38978-38984.

[23] J. Tian, L. Liu, S. Xia, Y. Diao, F. Lu, Optoelectronic properties of two-dimensional GaN adsorbed with H, N and O: A first-principle study, Physics Letters A, 383 (2019) 3018-3024.

[24] H. Gao, H. Ye, Z. Yu, Y. Zhang, Y. Liu, Y. Li, Point defects and composition in hexagonal group-III nitride monolayers: A first-principles calculation, Superlattices and Microstructures, 112 (2017) 136-142.

[25] R. González, W. López-Pérez, Á. González-García, M.G. Moreno-Armenta, R. GonzálezHernández, Vacancy charged defects in two-dimensional GaN, Applied Surface Science, 433 (2018) 1049-1055.

[26] J. Li, H. Liu, Magnetism investigation of GaN monolayer doped with group VIII B transition metals, Journal of Materials Science, 53 (2018) 15986-15994.

[27] Q. Zhao, Z. Xiong, L. Luo, Z. Sun, Z. Qin, L. Chen, N. Wu, Design of a new two-dimensional diluted magnetic semiconductor: Mn-doped GaN monolayer, Applied Surface Science, 396 (2017) 480-483.

[28] Z.Y. Al Balushi, K. Wang, R.K. Ghosh, R.A. Vilá, S.M. Eichfeld, J.D. Caldwell, X. Qin, Y.-C. Lin, P.A. DeSario, G. Stone, S. Subramanian, D.F. Paul, R.M. Wallace, S. Datta, Joan M. 
Redwing, J.A. Robinson, Two-dimensional gallium nitride realized via graphene encapsulation, Nature Materials, 15 (2016) 1166-1171.

[29] N.A. Koratkar, Two-dimensional gallium nitride, Nature Materials, 15 (2016) 1153-1154.

[30] Y. Chen, K. Liu, J. Liu, T. Lv, B. Wei, T. Zhang, M. Zeng, Z. Wang, L. Fu, Growth of 2D GaN Single Crystals on Liquid Metals, Journal of the American Chemical Society, 140 (2018) 1639216395.

[31] Y. Cao, V. Fatemi, A. Demir, S. Fang, S.L. Tomarken, J.Y. Luo, J.D. Sanchez-Yamagishi, K. Watanabe, T. Taniguchi, E. Kaxiras, R.C. Ashoori, P. Jarillo-Herrero, Correlated insulator behaviour at half-filling in magic-angle graphene superlattices, Nature, 556 (2018) 80-84.

[32] Y. Cao, V. Fatemi, S. Fang, K. Watanabe, T. Taniguchi, E. Kaxiras, P. Jarillo-Herrero, Unconventional superconductivity in magic-angle graphene superlattices, Nature, 556 (2018) 43-50.

[33] N.B. Le, T.D. Huan, L.M. Woods, Interlayer Interactions in van der Waals Heterostructures: Electron and Phonon Properties, ACS Appl. Mater. Interfaces, 8 (2016) 6286-6292.

[34] S. Deng, L. Li, P. Rees, Graphene/MoXY Heterostructures Adjusted by Interlayer Distance, External Electric Field, and Strain for Tunable Devices, ACS Applied Nano Materials, 2 (2019) 3977-3988.

[35] S. Deng, Y. Zhang, L. Li, Study on electronic and optical properties of the twisted and strained MoS2/PtS2 heterogeneous interface, Applied Surface Science, 476 (2019) 308-316.

[36] F. Wooden, Optical Properties Of Solids, in, Academic Press New York and London, 1972.

[37] K. Okamoto, Fundamentals of Optical Waveguides (Second Edition), in, Academic Press, Burlington, 2006.

[38] J.F. Muth, J.H. Lee, I.K. Shmagin, R.M. Kolbas, H.C.C. Jr., B.P. Keller, U.K. Mishra, S.P. DenBaars, Absorption coefficient, energy gap, exciton binding energy, and recombination lifetime of GaN obtained from transmission measurements, Applied Physics Letters, 71 (1997) 2572-2574.

[39] K.S. Novoselov, A. Mishchenko, A. Carvalho, A.H. Castro Neto, 2D materials and van der Waals heterostructures, Science, 353 (2016) aac9439. 


\section{Chapter5: Conclusion}

By applying first principle-based calculation, I have investigated the. Properties of many materials Firstly, I investigate the electron transport and thermoelectric performance of TBGNRJs for $0^{\circ}, 21.8^{\circ}, 38.2^{\circ}$ and $60^{\circ}$ rotation angles by the first principles calculation. It is found that the $I-V$ curves of $0^{\circ}, 21.8^{\circ}$ and $38.2^{\circ}$ rotation angles exhibit behavior similar to $\mathrm{p}-\mathrm{n}$ junctions. However, the $60^{\circ}$ TBGNRJs is different, which exhibits an $I-V$ curve similar to a transistor. NDR is shown in TBGNRJs at $21.8^{\circ}$ and $38.2^{\circ}$ rotation angles under $\pm 0.2 \mathrm{~V}$ bias voltage. High $Z T$ values of 2.0, 2.7 and 6.1 have been achieved at $-0.25 \mathrm{eV}$, $0.33 \mathrm{eV}$ and $0.68 \mathrm{eV}$ for the $21.8^{\circ}$ rotation angles at $300 \mathrm{~K}$. It is interpreted that the reason of high $Z T$ values of $21.8^{\circ}$ TBGNRJ is due to its wider bandgap, transmission gap, electrical conduction gap and a strong reduction of thermal conductance for the TBGNRJs compared with the single GNR. Moreover, the phonon contribution to thermal conductance is much more than that of electrons in $Z T$ values of 2.0 and 2.7 in the $21.8^{\circ}$ TBGNRJs. At the $Z T$ value of 6.1, the electron contribution to thermal conductance is comparable to that of phonons.

Secondly, electronic and optical properties of the GaN bilayer structure with rotation angles $0^{\circ}, 27.8^{\circ}, 38.2^{\circ}$ and $46.8^{\circ}$ were simulated with the density functional theory. According to the first principle simulation, values of bandgap, absorption coefficient, extinction coefficient, reflectivity and the refractive index vary with the rotation angles. The two vdW bonded GaN monolayers have been found to exhibit wide bandgap, which is further increased by twisting these two layers at certain optimized angles. It is also discovered that the refractive index of the bilayer structure is much smaller compared with its bulk form. he absorption coefficient has significantly reduced at all twisting angles including $0^{\circ}$. The calculated optical absorption coefficient $\left(\sim 10^{2} \mathrm{~cm}^{-1}\right)$ of the bilayer $\mathrm{GaN}$ is much lower than that of the bulk GaN $\left(\sim 10^{5} \mathrm{~cm}^{-1}\right)$, reducing the power loss 
due to the absorption. Bilayer structure brings the advantage of increased optical bandgap and reduced optical power loss due to significant reduction of both the absorption coefficient and refractive index, which makes these bilayer GaN structures more suitable for monolithic integration of power electronic devices and optical devices.

Finally, by going through all the four years, I not only gained a lot of knowledge about nanotechnology, but also learned the skills to carry on a project. I learned and practice how to use matlab, quantumATK toolkit then did simulation and calculation with these tools. This thesis presents what I have learnt in my PHD career. I mainly focus on using first principle methods to simulate and discover the property of new semiconductor materials and two outstanding instants are Graphene and GaN. I have two papers published about these two materials and hope to get more in the future.

\section{Possible Further Study}

First principle-based calculation is a very useful method in investigating the properties of materials in nano scale. By applying DFT calculation, even the properties of unknown material could be speculated. This is quite important in solid state physics study.Both Graphene and GaN are quite popular and have great potential. They are the material leading to change the world. If possible, more research should be done to discovery more of the property of these materials to make breakthrough in related products.

\section{Acknowledgements}

Authors are grateful for Prof Lijie Li, who is the supervisor of author, for the patience and instruction given. 